\title{
EYE "R" GLASSES: DEVELOPMENT OF AN INFRARED SENSOR SYSTEM FOR DETECTING THE HUMAN BODY
}

\author{
A Thesis \\ presented to \\ the Faculty of California Polytechnic State University, \\ San Luis Obispo
}

\author{
In Partial Fulfillment \\ of the Requirements for the Degree \\ Master of Science in Electrical Engineering
}

by

Rick Wong

June 2013 
(C)2013

Rick Wong

ALL RIGHTS RESERVED 
TITLE:

AUTHOR:

DATE SUBMITTED:

COMMITTEE CHAIR:

COMMITTEE MEMBER:

COMMITTEE MEMBER:
Eye "R" Glasses: Development of an Infrared Sensor System for Detecting the Human Body

Rick Wong

June 10, 2013

Dr. Vladimir Prodanov, Assistant Professor Electrical Engineering Department

Dr. John Y. Oliver, Assistant Professor Electrical Engineering Department

Dr. Tina Smilkstein, Assistant Professor Electrical Engineering Department 


\begin{abstract}
Eye "R" Glasses: Development of an Infrared Sensor System for Detecting the Human Body
\end{abstract}

\title{
Rick Wong
}

Throughout the years, sensors have been an integral part of automation, alert, and medical systems. Many of these systems measure physiological characteristics of the human body to alert themselves of their current conditions. Drowsy driver systems, for instance, measure the eyes and facial movements with a camera to determine if the driver is falling asleep at the wheel. Electroencephalography (EEG), electrooculography (EOG), and electromyography (EMG) employ electrodes on the human body to measure electrical activity of a patient's REM sleep cycle patterns. Pulse oximeters use optical light through a process called photoplethysmography (PPG) to measure heart rate.

As diverse as these all may be, this thesis attempts to prove infrared technology as a single, resourceful, and inexpensive method for implementing all the aforementioned systems. This thesis specifically explores the development of Silicon Laboratories' Si1143 Infrared Proximity/Ambient Light Sensor into a pair of eye tracking/heart rate detecting glasses. Through the use of a LabVIEW interface, a novel algorithmic solution is also presented to classify the eye movements and detect the heart rate signal.

The results from the tests and calculations show that the Si1143 sensor can detect eye movements using only a $52 \mu \mathrm{W}$ of power. The novel algorithm can also classify the blinking motions robustly, but the algorithm starts to fail when additional motions are added. The results also show that the Si1143 sensor can detect heart rate using Reflection PPG method, but imprecise placement of the sensor on the glasses will render the measurement useless. This thesis concludes that Si1143 sensor is sensitive enough to track the human eye and measure the heart rate, but further work is required to make it robust. 


\section{ACKNOWLEDGMENTS}

First and foremost, I would like to thank Dr. Vladimir Prodanov for being my advisor throughout this thesis. Even though there was so many times where I felt like giving up, he was always there to reassure me of my abilities as an electrical engineering student. Thank you for not only teaching me EE material but also how to be EE material. I would also like to thank my committee members, Dr. John Oliver and Dr. Tina Smilkstein, for being great teachers to me throughout the years. My college education would not be the same without you.

I'd also like to thank my fellow colleague, friend, and housemate, Kenneth Chee, for encouraging me, helping me, and lending me his workshop tools. I literally could not have finished without you. I also want to thank all my friends that encouraged me to stay strong and finish my studies.

Lastly, I'd like to thank my family members for all the physical, emotional, spiritual, and financial support throughout these long six years. It's been a difficult journey to get through, but it would have been an impossible feat without you. 
LIST OF TABLES ............................................................................................................ viii

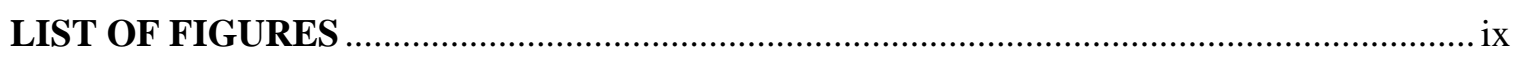

CHAPTER 1. INTRODUCTION ……………………….................................................. 1

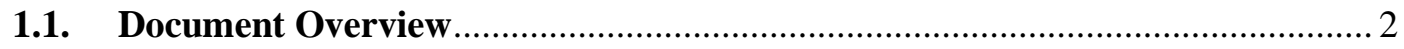

CHAPTER 2. BACKGROUND AND MOTIVATION ………………………………...... 3

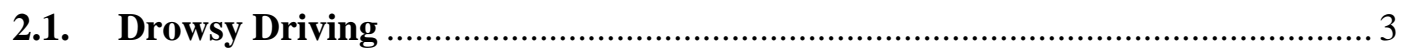

2.2. REM Sleep Cycle Detection ………………………………………………….. 7

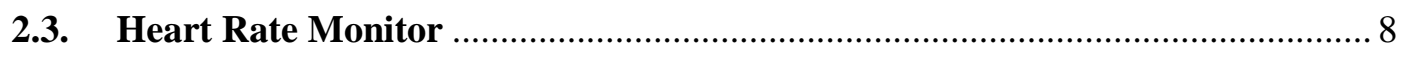

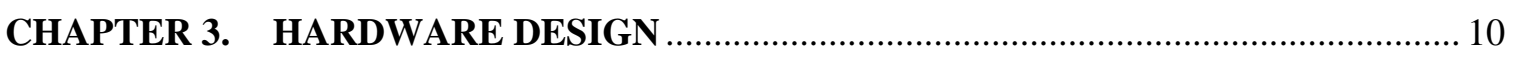

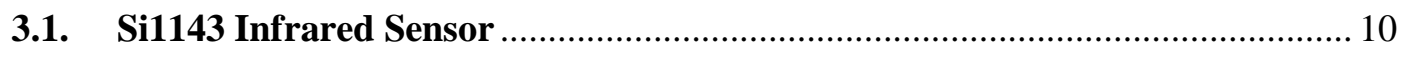

3.2. Infrared LED Selection ........................................................................... 12

3.2.1. Safety Concerns.................................................................................... 17

3.2.2. Power Consumption of OSRAM SFH 4056 LED...................................... 21

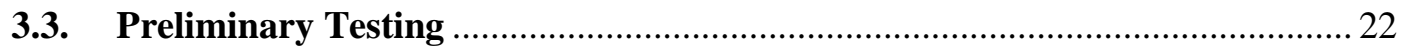

CHAPTER 4. GLASSES PROTOTYPE DESIGN …………………………………........ 28

4.1. Circuit Schematic and Component Selection …………………………….... 28

4.2. Assembly and Placement Procedure ............................................................ 31

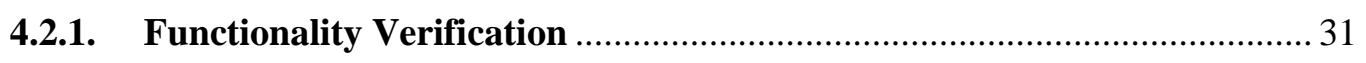

4.2.2. Glasses Construction ……………………………………………………. 32

4.3. Software and Programming Development ........................................................ 36

4.4. Initial Experimental Results and Analysis.......................................................... 39

4.5. Printed Circuit Board Design ……………………………………………... 41

4.6. PCB Assembly Procedure …………………………………………….... 42

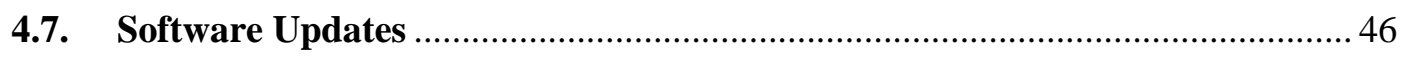

4.8. Final Experimental Results and Analysis …………………………………....5 50

4.8.1. Experimental LED Power Consumption .................................................... 53

CHAPTER 5. HEART RATE SENSOR …………………………………………. 55

5.1. First Prototype Test Setup ……………………………………………….... 55

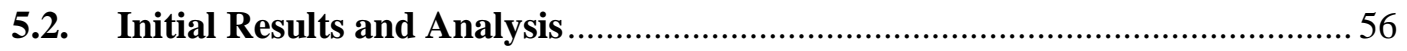

5.3. Second Iteration of Software Design for Heartbeat Sensor …….......................59 


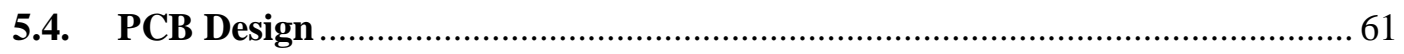

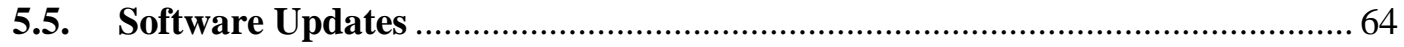

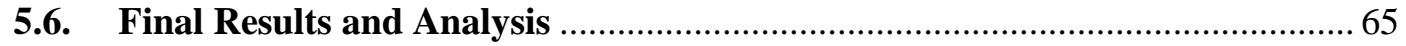

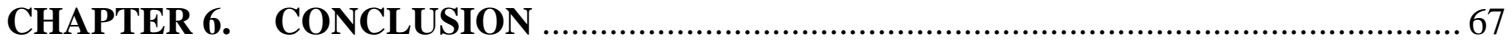

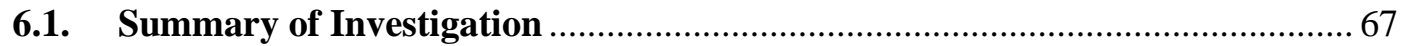

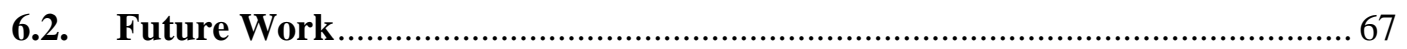

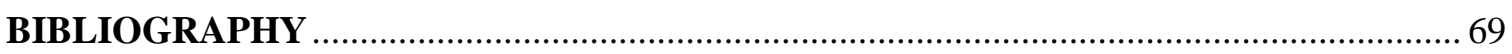

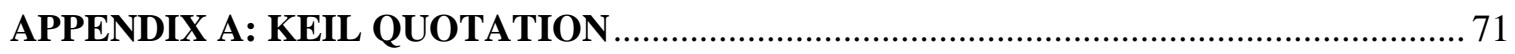

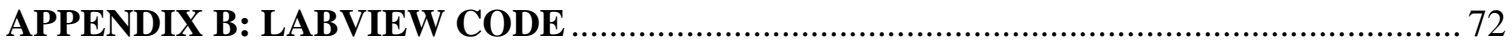

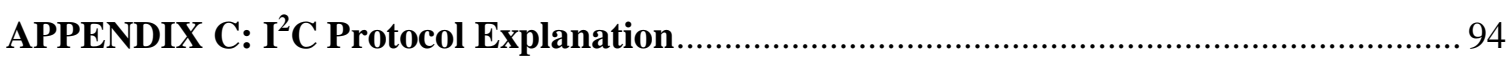




\section{LIST OF TABLES}

Table

Page

Table 1. Performance Comparision between Three Different Infrared Sensors - This table shows that the Si1143 outperforms the SFH7743 and the TSL2572 in every category.

Table 2. Typical Irradiance Design Targets [10] - These minimum irradiance values at the sensor are based on current illumination conditions, the ADC integration time, the IR photo diode used, and the HSIG bit setting 16

Table 3. Actively Measuring Time Performance Characteristic [9] - These are typical measuring times for each type of measurement taken by the Si1143. As more parameters are measured, the measuring time increases.

Table 4. LED Pulse Width Performance Characteristics [9] - The typical and max time for each LED pulse width are shown to be 25.6 microseconds and 30 microseconds respectively.

Table 5. Reverse and Forward Coefficients for a 7th Order Butterworth Highpass IIR Filter (Normalized Cutoff Frequency $=0.1$ ). This filter removed the wandering baseline signal from the waveform to provide a better implementation of the peak detector. 49 


\section{LIST OF FIGURES}

Figure

Page

Figure 1. Areas to Measure Pulse around the Ear - The Superficial temporal artery around the ear is the site where the Si1143 sensor will attempt to measure heart rate. (from Wikimedia commons) [16]......

Figure 2. Size Comparison of Si1143 chip to a Dime - The Si1143 proximity/ambient light sensor is approximately 63 times smaller than the U.S. dime. 10

Figure 3. irLED Radiant Intensity vs Half-Angle - The half angle of an infrared LED is the angle in which the radiant intensity is half of the max value or half of the value in the axial direction. 14

Figure 4. Design of Concept Glasses - This sketch shows the approximate placements of the infrared LEDs and Si1143 proximity/ambient light sensor to track eye movements. 14

Figure 5. Side View of Concept Glasses - This sketch shows how the infrared LED will shine light on the eye. For ideal use of the infrared LED, the half angle should cover the entire eye.... 15

Figure 6. OSRAM SFH 4056 Radiation Characteristics [13] - The radiant intensity decreases greatly as the angle increases. The half angle is shown to be at $22^{\circ}$.....

Figure 7. OSRAM SFH 4056 Relative Spectral Emission [13] - The graph displays the radiant intensity emitted across the spectrum of wavelengths. The wavelength at peak emission is shown as $860 \mathrm{~nm}$.

Figure 8. Si1143 Development Board - The board features three OSRAM SFH 4056 Infrared LEDs, the Si1143 sensor, and the C8051F800 microcontroller.

Figure 9. Measurement Time between each LED pulse - The time period between each LED pulse measures $3.27 \mathrm{~ms}$. The speed at which the Si1143 measures data is $306 \mathrm{~Hz}$.

Figure 10. Measurements of LED1 and LED2 Pulse Width - Channel 1 measures the voltage drop across LED1 while Channel 2 measures the voltage drop across LED2. The waveform shows a measured pulse width of 27 microseconds.

Figure 11. Left Glance Detection of the Left Eye - As the left eye makes left glances, the counts on the graph increase and then decrease. The graph shows five of these left glances. 26

Figure 12. Screenshot of Silicon Laboratories IDE - The highlighted portion in red indicates that the compiled code size limit has been reached.

Figure 13. Si1143 Circuit Schematic [12] - A separate voltage rail design was used to ensure enough current could be supplied to the infrared LEDs. .28 
Figure 14. LED Driver Circuit on the Si1143 [12] - The Si1 143 controls the amount of current pulsed through the irLED.

Figure 15. Si1143 Sensor on a QFN-10 Breakout Board - The breakout board enabled access to the pins of the Si1143 sensor for prototype testing..... 31

Figure 16. Prototype Design Test Setup on Breadboard - The circuit was first tested on this breadboard setup to verify functionality. 32

Figure 17. Real D 3D Glasses - These glasses provided the framework for initial prototyping. The names for each part of the glasses are also defined.

Figure 18. Dremel and Sanding Drum - These tools were used for to cut the breakout board into shape for placement onto the Real D 3D Glasses.

Figure 19. 3M Breathing Apparatus - This mask prevented dangerous FR-4 dust from being inhaled when cuts were made to the PCB board.

Figure 20. Assembled Glasses Prototype - This prototype was the first design used to test the concept of infrared eye tracking.

Figure 21. Instamorph Moldable Plastic - This moldable plastic was used to form the nosepiece and earpiece adjustments to the initial and final prototype glasses.

Figure 22. Screenshot of LIFA Firmware Sketch - This sketch enabled LabVIEW to interface with the Arduino.

Figure 23. Host Command Structure - This figure illustrates the hierarchal structure of how the computer communicates with the Si1143 sensor.....

Figure 24. Flowchart of LabVIEW Program - This flowchart outlines the main protocol for measuring and acquiring data from the Si1143 sensor.

Figure 25. Eye Tracking LabVIEW Front Panel - The front panel shows the acquisition and plotting of the data in real time. The current drive for each LED was set at $5.6 \mathrm{~mA}$ during initial test runs.

Figure 26. Blink Test Run - The graph shows three proximity sensing measurements taken when the user blinks. Each blink corresponded to a sudden spike in each of the PS channels.

Figure 27. ExpressSCH Schematic of Si1143 Circuit - This schematic was linked to the ExpressPCB layout for easier routing. Multiple LED components were added to simulate multiple pad placements around the rim of the glasses. 
Figure 28. ExpressPCB Layout of Glasses Rim - The layout of the rims measured $16.3 \mathrm{~cm}$ in length and $6 \mathrm{~cm}$ in height. Multiple pads for the infrared LEDs were placed around the rims for multiple test configurations.

Figure 29. Fabricated PCB of Glasses Rim - This image shows the top layer of the fabricated PCB board.

Figure 30. 1/16" Cut-off Wheel - This attachment was used instead of the sanding drum to cut out the fabricated PCB board.

Figure 31. Schlage Jewelry Box Hinge - The hinge provided pivotal motion of the temple pieces.

Figure 32. Devcon Home 5-minute Epoxy - This adhesive attached the hinge onto the PCB material. It was used as substitute for drilling screws into the PCB.

Figure 33. Typical Solder Profile - The temperature profile was roughly followed to ensure proper assembly of the components. This was done manually through the use of a hot air gun...45

Figure 34. Assembled PCB Glasses Prototype - The final prototype was used to re-confirm initial findings and test the classification algorithm. 46

Figure 35. Left Glance, Right Glance, and Blink Test - Each movement shows a unique set of characteristics in each of the proximity channels. By identifying each of these, the correct movement can be classified.

Figure 36. Idealized PS Waveform for Left Glance, Right Glance, and Blink Movement - An idealized waveform was created based on previous observations. It served as a test basis for creating the classification algorithm.

Figure 37. Graphical Algorithmic Solution - This figure visually displays the steps taken by the algorithm to identify each eye movement.

Figure 38. Successful Test Run of Tracking Eye Movement - This front panel VI shows a successful run of the measured PS channels on the left and the classified motion on the right. The test run started with five blinks, followed by a left glance and right glance, and ended with five more blinks.

Figure 39. Unsuccessful Test Run of Eye Tracking Movement - This front panel VI shows an unsuccessful run of the measured PS channels on the left and the classified motion on the right. The eye movements for the test run are as follows: one blink, a left glance and right glance, followed by two blinks, a left glance and right glance, and ended with two blinks .... 
Figure 40. Analysis of Unsuccessful Test Run - Error resulted from the left and right glance detection algorithm. The right graph displays a visual explanation for the error.

Figure 41. Voltage Drop Measurement across Pulsed LED. The cursors show a measured forward voltage drop of $1.6 \mathrm{~V}$ across LED1 of the PCB prototype glasses when driven. This value will be used to calculate experimental LED power consumption. .53

Figure 42. Time of Pulsed LED Measurement. The cursors measure the LED1 pulse width of the PCB prototype glasses occurring in 28 microseconds. This value will be used to calculate experimental LED power consumption.

Figure 43. Time Period between Each Pulsed LED Measurement. The cursors measure the time between each LED pulse to be 86 microseconds. This value will be used to calculate experimental LED power consumption.

Figure 44. Reflection PPG Heart Rate Sensor Test Setup - The sensor is on the same plane as the LED and captures the reflectance off the blood vessels. Long wires were attached to this test setup to enable sensor placement on the Superior temporal artery.

Figure 45. Transmission PPG (left) and Reflection PPG (right) - The transmission PPG captures the volumetric change of the blood vessels through the transmitted IR wave while the reflection PPG captures the volumetric change through the reflected IR wave.

Figure 46. LabVIEW Front Panel VI for Heart Rate Sensor - This front panel interface shows the graph of an initial test run of the design.

Figure 47. Heart Rate Test on Index Finger via Transmission Before Exercise - The graph shows a measurement of the PS1 channel on the index finger. The heart rate was measured through the transmission PPG method before mild exercise was performed. The results show an $81 \mathrm{bpm}$ rate.

Figure 48. Heart Rate Test on Index Finger via Transmission After Exercise - The graph shows the an increase in frequency on the PS1 channel after mild exercise was performed. The same transmission PPG method was used to show an increased heart rate of $129 \mathrm{bpm}$.

Figure 49. Heart Rate on Index Finger via Reflection PPG - The graph shows the results of a reflection PPG test run on the index finger. The waveform exhibits a consistent wave pattern as in the transmission PPG case. .58

Figure 50. Heart Rate on Superior Temporal Artery via Reflection - The graph shows the results of a reflection PPG test run when the sensor is placed on the Superior temporal artery. The waveform shows that it is possible to get a reading of the heart rate. 
Figure 51. Moving Average Hysteresis Comparator - This circuit was implemented in the program to detect the peaks of the waveform and convert them to a digital signal.

Figure 52. Front Panel of Heart Rate Test Run with Digitized Count - This front panel image shows the result of implementing the hysteresis comparator circuit in LabVIEW. Each corresponding digital high output represents when the heart beats.

Figure 53. ExpressSCH Schematic for Left Temple Piece - This schematic is modified from the eye tracker circuit to enable multiple placements of the sensor around the left temple piece of the PCB glasses.

Figure 54. ExpressPCB Layout of Left Temple Piece - This layout shows the pad placements and routing for all the components specified in the left side heart rate schematic. 62

Figure 55. ExpressSCH Schematic of Right Temple Piece - This schematic is modified from the eye tracker circuit to enable the use of eight sensors to measure heart rate.

Figure 56. ExpressPCB Layout of Right Temple Piece - This layout shows the pad placements and routing for all the components specified in the right side heart rate schematic.

Figure 57. PCB Test Run of Heart Rate Sensor - This front panel image captures a typical test run for the final PCB prototype design. The graph on the right shows no distinguishable, consistent waveform as seen in previous tests. 


\section{CHAPTER 1. INTRODUCTION}

As technology continues to grow, automation and alert systems are becoming more integrated into the human lifestyle. There are a number of devices and systems out on the market today that help consumers with performing their simple daily tasks. For example, Ford has recently released cars like the 2013 Ford Fiesta with Active Park Assist. These cars employ the use of ultrasonic sensors to measure the proximity of nearby objects, calculate optimal steering angles, and perform the necessary steering maneuvers to aid in parallel parking a car. Ford is also using this same technology to engineer the traffic jam assist, a system that can optionally take over driving when the stop and go traffic is detected.

The development of sensors has also been helpful in measuring the human body. One example is the medical infrared thermometer that quickly senses the body's temperature when measured at the forehead or earlobe. Accurately sensing the human body unlocks numerous possibilities for integrating that data with automated, alert, or recognition systems.

With the emergence of all these technologies, it is important to analyze each one and compare the advantages and disadvantages of each system. This thesis explores the Si1143 Infrared Proximity/Ambient Light Sensor as a single, inexpensive technology to implement a drowsy driving detection system, REM sleep cycle detection system, and heart rate sensor system. All of these systems will be realized by creating a pair of wearable glasses that can track the human eye and measure the body's heart rate. 


\subsection{Document Overview}

This document covers the progression of this thesis, starting from the initial problem to the final design. This chapter introduces the goal that this thesis is trying to achieve. Chapter 2 lays out the background knowledge of existing problems and systems related to drowsy driving, REM sleep detection, and heart rate measurements. Chapter 3 talks about the preliminary testing of the Si1143 Infrared Sensor chip to have a proof of concept for the glasses as well as the proposed theory and safety concerns with infrared. Chapter 4 goes into the development and analysis of the eye tracking glasses, from initial prototype of the hardware and software to the final prototype. Chapter 5 covers the design of the heart rate sensor and an analysis of its effectiveness. Finally, Chapter 6 provides a conclusion to this thesis and lists ideas for future works. 


\section{CHAPTER 2. BACKGROUND AND MOTIVATION}

This chapter focuses on the background and motivations for creating a pair of infrared sensing glasses. Each section describes the problem and then gives current methods that are used to solve these problems. Finally, the end of each section concludes with how the proposed system will provide an alternative solution to the problems.

\subsection{Drowsy Driving}

Statistics on traffic accidents show that human error is a major cause for crashes and fatalities on roads and highways. Drowsy driving impairs one's judgment and reaction time as much as alcohol and drugs do and is therefore categorized as another form of distracted driving. The number of accidents related to driver drowsiness is reported by the NHTSA to be about 56,000 crashes annually. From these crashes, 40,000 nonfatal injuries and 1,550 fatal injuries are reported. Overall, 37\% of the driving population says they have nodded off for at least a moment or fallen asleep while driving at some time in their life. Given the nature of drowsy driving, it should be noted that these statistics are usually underreported [1].

Many factors contribute to these driving performances such as the amount of driving, time of day, sleep deprivation, inattention, monotonous driving environment, heat, noise, and weather conditions. In a case-control study of 571 drivers involved in road crashes [2], a risk of a car crash leading to serious injury or death substantially increases when the driver is driving sleepy, driving after five hours of sleep or less, and driving between 2:00 a.m. and 5:00 a.m. Among those most affected by drowsy driving are commercial motor vehicle truck drivers. Given the long hours of driving and usual 
night driving, it is estimated that 1,200 deaths and 76,000 injuries can be attributed to fatigue related crashes. The cost of these crashes is estimated at $\$ 12.4$ billion per year [3]. With all these statistics in mind, it is imperative to design and create a system that can monitor and alert drivers in danger of a drowsy driving episode.

Drivers who are in a state of drowsiness usually produce various visual characteristics. The first category of characteristics would be physiological, which would include yawning frequency, eye-blinking frequency, eye-gaze movement, drooping eyelids, head movement, facial expressions, electroencephalogram (EEG) variations, and electrocardiogram (ECG) variations $[2,4]$. Another category of characteristics uses driving control information to identify manifestations of drowsiness. This information includes speed, acceleration, lane keeping information, steering wheel angle, etc. [2]. There are advantages and disadvantages to using both categories of classification. With physiological information, the data is almost directly linked to the driver's alertness status and therefore, systems can provide a quick response. However, some forms of measurement like EEG or ECG would be intrusive by nature. Driving control information, on the other hand, would require no data collected from monitoring the individual and would be an easier system to develop and implement on each vehicle. There are several systems already on the market that employ alerts using these features. The 2010 Mercedes Benz E-Class is equipped with Attention Assist which monitors and observes the driver's steering behavior across 70 different parameters. One of the monitors is a highly sensitive steering angle sensor that detects patterns of minor steering corrections. When the steering wheel movements match a drowsy driver profile, the system emits an audible and visible warning to alert the driver. Volvo also offers the 
Driver Alert Control System which uses a camera to monitor the road for instances of straying off lane markers. As successful as these kinds of systems may be, the data is still indirectly linked to the status of the driver and could potentially cause false alarms. They may also not provide adequate warning time as these systems alert the driver after the driver has committed a drowsy driving pattern.

Most, if not all systems, presented in other works use a camera-based approach towards capturing the data and processing it for analysis. The advantage to using a camera is that the system would be a non-intrusive form of measurement. Along with this approach, several factors such as illumination conditions and changing facial positions present some difficulties in achieving a proper image. In order to alleviate this problem, image processing and infrared illumination are used to achieve a better result. Flores et al. [4] present a system that analyzes greyscale nighttime images taken in infrared illumination under real driving conditions. The main principle behind locating the position of the eye in this system is through the bright pupil effect. The bright pupil effect occurs when incident infrared light reflects off the pupil, causing bright spots in the image much like the red-eye effect. By using three methods of processing on the original image, the system creates a more robust algorithm for detecting these bright spots. Once the eyes are detected and tracked, the percentage of eye closure over time (PERCLOS) index is used as an indicator to the drowsiness or fatigue level of the driver. PERCLOS is defined as the proportion of time the eyes are closed $80 \%$ or more for a specified time interval. Mehrubeoglu et al. [5] present another system where a National Instruments Smart Camera is used to capture the image of the user's eyes. The eyes in this image are detected by using template matching. Template matching takes a pre-existing template of 
user's eyes and uses a matching metric to measure the similarity between the two images. If the eye is detected, the image is then cropped to focus only on the eye and reduce the processing area. This new region of interest then undergoes edge detection to plot points around the eye and record the coordinates of its position.

Both of these systems show a detection and classification success rate of at least $90 \%$. However, the disadvantage of both systems is that detection performance decreases significantly when the face looks away. Another disadvantage of Flores et al.'s system is that constant IR illumination is needed when tracking the eye. The constant illumination results in more power consumption. By wearing a pair of glasses that tracks the eye wherever the user looks, it eliminates these disadvantages. According to Jobson Research in 2005 , over $61 \%$ of the US population needed some eyewear for vision correction [6]. These statistics give additional motivation for exploring glasses as a viable option in implementing a drowsy driving detection system.

The idea behind the glasses is to place an infrared sensor and three infrared LEDs on the rim of the glasses. Each infrared LED would be pulsed on one at a time (which would save on power consumption) and the sensor would take measurements from the reflectance. Since the LEDs are placed in three different locations and the reflectance off each part of the eye is different [3], the sensor will pick up three unique measurements associated with the position of the eye. An algorithm is then implemented to process those measurements and correspond them to a specific eye movement.

There are three main eye movements explored in this thesis. They are blinking, looking to the left, and looking to the right. Blinking is the act of closing the eyelids 
momentarily either voluntarily or involuntarily. Blinking usually occurs in a very short amount of time and should not be confused with closing the eyes for a long period of time and then opening them. Left glances occur when the eye is in a neutral position, moves and stays to the left for a period of time, and then back to the neutral position. The neutral position is defined as looking straight. Conversely, right glances are the same but in the direction to the right.

\subsection{REM Sleep Cycle Detection}

Rapid Eye Movement (REM) is a stage of an individual's sleep cycle where the eye exhibits rapid and random movements. During this stage, there is an increase in respiration, heart rate, brain activity, and muscle relaxation. Research also shows that the REM stage is the period for which dreaming occurs. Several studies and theories have gone into the importance of REM sleep. Yaso et al. [7] presents a study where subjects feel more awake comfortable when woken up from REM sleep than in non-REM sleep. Iranzo et al. [8] presents a study where REM behavior Disorder (RBD) may be early indications for neurodegenerative diseases such as Parkinsons disease. RBD is a parasomnia characterized by dream-enacting behaviors related to unpleasant dreams and loss of muscle atonia during REM sleep. By determining any irregularities during the REM sleep cycle, researchers can diagnosis if a patient exhibit RBD. Therefore, detection of REM sleep cycle in individuals is an important step in further applications of the medical and health fields.

Currently, electroencephalography (EEG), electrooculography (EOG), and electromyography (EMG) provide the best method in detecting REM sleep cycles. This 
detection method involves placing many electrodes on the patient. Other works such as Yaso et al. [7] attempt to bypass this intrusive form by classifying REM sleep cycle from variations in the heart rate. Their studies have shown a $79 \%$ accuracy rating in their detection method versus traditional methods. The glasses in this thesis will attempt to detect REM sleep cycle by measuring the protrusions of the cornea when the eyes are closed during sleep. From these measurements, an algorithm can be made to process and classify each movement.

\subsection{Heart Rate Monitor}

It is a well-established fact that blood flow and heart rate are key indicators of a person's health. Average heart rates of healthy individuals at rest usually lie within the range of $60-80$ beats per minute. There currently exist several different methods to measure the heart rate. An electrocardiography (ECG or EKG) measures the electrical activity of the heart by placing electrodes across the chest of the body.

Photoplethsmography (PPG) measures the volumetric change of the arterial vessels by passing light through and sensing the transmitted or reflected signals. Lastly, traditional methods of manually measuring the pulse around the wrist or neck region also work.

There are many sites around the body to measure the heart rate. The most common ones are around the neck or wrist. The regions of interest in this thesis are on the index finger and around the superficial temporal artery as shown in Figure 1. These sites are tested with the Si1143 sensor through reflection and/or transmission PPG to verify proof of concept. 


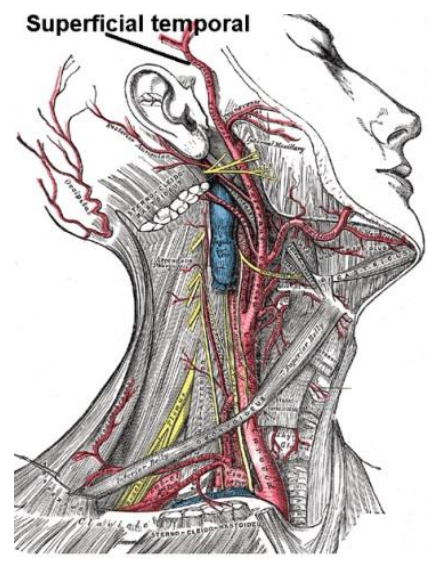

Figure 1. Areas to Measure Pulse around the Ear - The Superficial temporal artery around the ear is the site where the Si1143 sensor will attempt to measure heart rate. (from Wikimedia commons) [16]

After verification, the sensors and infrared LEDs will then be placed along the temple pieces of the glasses where it would lie directly above the superficial temporal artery. Through reflection PPG, the Si1143 proximity/ambient sensor would detect the volumetric change in blood flow when infrared light from the irLEDS reflect off the blood vessel. This volumetric change would occur periodically and would represent the times where the heart beats. The data from the waveform would then be processed, digitized, and shown to the user. 


\section{CHAPTER 3. HARDWARE DESIGN}

This chapter discusses the specifications of the hardware used for this thesis. It will talk about the operational characteristics of the Si1143 infrared sensor as well as the selection process for the infrared LED. Infrared safety and power consumption are also mentioned. Finally, the chapter ends with the initial testing of the sensor using the Si1143 Development Board.

\subsection{Si1143 Infrared Sensor}

The Si1143 is an active optical reflectance proximity detector and ambient light sensor. The chip outline spans $2 \times 2 \mathrm{~mm}$ and comes in QFN packaging design [9]. Figure 2 below shows a size comparison to a dime.

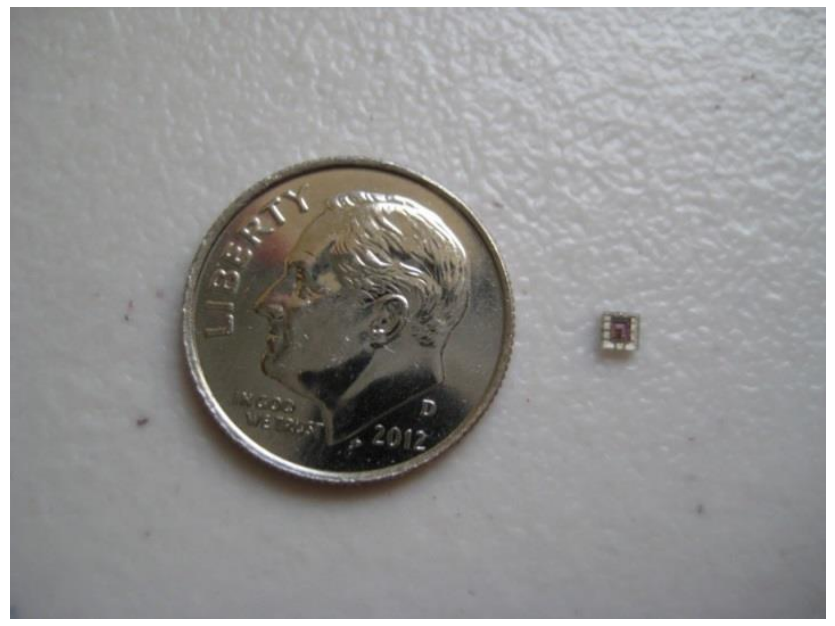

Figure 2. Size Comparison of Si1143 chip to a Dime - The Si1143 proximity/ambient light sensor is approximately 63 times smaller than the U.S. dime.

The sensor features low power consumption with average current consumption of $9 \mu \mathrm{A}$ and a low standby current of less than $500 \mathrm{nA}$. It has three independent LED drivers with pulse widths of $25.6 \mu \mathrm{S}$ each and each driver can be adjusted to a variable current 
drive setting. It operates up to 128 klux which is the equivalent to direct sunlight and it has a high reflectance sensitivity of less than $1 \mu \mathrm{W} / \mathrm{cm}^{2}$. The sensor itself communicates via $\mathrm{I}^{2} \mathrm{C}$ interface with speeds of up to $3.4 \mathrm{Mbps}$ and is controlled through a number of registers and command protocols [9]. When compared with other proximity sensors such as OSRAM SFH 7743 Digital Proximity Detector or TAOS TSL2572 Light-to-Digital Converter as shown in Table 1, the Si1143 outperforms in terms of power consumption, range in luminance, number of channels, and current driving capacity.

\begin{tabular}{|c|c|c|c|c|c|}
\hline Sensor & $\begin{array}{c}\text { Current } \\
\text { during } \\
\text { Active } \\
\text { Mode } \\
(\boldsymbol{\mu A})\end{array}$ & $\begin{array}{c}\text { Current } \\
\text { during } \\
\text { Standby } \\
\text { Mode } \\
(\boldsymbol{\mu A})\end{array}$ & $\begin{array}{c}\text { Maximum } \\
\text { Measurable } \\
\text { Illuminance } \\
(\mathbf{l x})\end{array}$ & $\begin{array}{c}\text { \# of } \\
\text { Measurement } \\
\text { Channels }\end{array}$ & $\begin{array}{c}\text { Maximum } \\
\text { Current } \\
\text { of Pulsed } \\
\text { LED } \\
(\mathbf{m A})\end{array}$ \\
\hline Si1143 & 9 & 0.5 & 128,000 & 3 & 359 \\
\hline SFH7743 & 45 & N/A & 1000 & 1 & 60 \\
\hline TSL2572 & 200 & 90 & 60,000 & 2 & N/A \\
\hline
\end{tabular}

Table 1. Performance Comparision between Three Different Infrared Sensors - This table shows that the Si1143 outperforms the SFH7743 and the TSL2572 in every category.

The Si1143 can be operated in one of the five operational modes at any one time. In Off Mode, the sensor is turned off when $\mathrm{V}_{\mathrm{DD}}$ is not connected to the power supply or when the $\mathrm{V}_{\mathrm{DD}}$ voltage is less than the VDD_OFF voltage of $1.0 \mathrm{~V}$. The sensor enters Initialization Mode when the power applied to $\mathrm{V}_{\mathrm{DD}}$ is greater than the minimum value. The start-up time takes approximately $25 \mathrm{~ms}$ and it is recommended that no $\mathrm{I}^{2} \mathrm{C}$ activity takes place during this time. The Si1143 spends most of the time in Standby Mode where it awaits commands from the host on the $\mathrm{I}^{2} \mathrm{C}$ bus.

From Standby Mode, the Si1 143 can enter into the two remaining modes of operation. In Forced Conversion Mode, the host processor sends a specific command to the sensor and polls it for the data measurement. The host can read an Ambient Light 
Source measurement when it sends the ALS_FORCE command, Proximity Sensing measurement when it sends the PS_FORCE command, or both measurements when it sends the PSALS_FORCE command to the command register. On the contrary, Autonomous Operation Mode allows measurements to be performed automatically without the host to send a specific command each time. As each PS and ALS measurement is made, the Sil143 updates the $\mathrm{I}^{2} \mathrm{C}$ registers with the data to be read. In addition to this, the Si1143 can interrupt the host when the PS or ALS measurement reaches a pre-set threshold. Measure rates and conversion frequencies for the PS and ALS measurements must be set up prior to enabling Autonomous Operation Mode.

When the Si1143 makes a proximity measurement, it is actually making two separate measurements back to back within $25.6 \mu \mathrm{S}$ of each other. Before irLED is turned on, the Si1143 makes a measurement of the ambient infrared light alone. When irLED is turned on, it makes a second measurement that captures both the target reflectance and the ambient infrared light. Both measurements are digitized to a count value through the internal analog to digital converter (ADC). The Si1143 subtracts these two values to report only the target reflectance in its output register.

\subsection{Infrared LED Selection}

Selection of the infrared LED is critical when determining the application of the system. From the Application Note 521 by Silicon Laboratories [10], there are three important concepts to understand when making an irLED selection. The first concept is radiant flux. Radiant flux is a measurement of light power and is expressed in watts. The amount of radiant flux is linked to the cost of an irLED. Therefore, the higher the radiant 
flux, the greater the cost will be. The next concept is the half-angle of an irLED. The half-angle is defined as the angle where the radiant intensity has dropped $50 \%$ from its max value (in the axial direction) measured with respect to the irLED's center emission line as shown in Figure 3. An irLED with a narrow half-angle would mean that it concentrates most of its power in a small region of space. The last concept is radiant intensity. Radiant intensity is the measurement of radiant flux per unity solid angle from a point light source and is expressed in watts per steradian. It is equivalent to power density, in the sense of measuring the amount of power in a given area. From these definitions, it is important to note the relations between the three concepts. Radiant Flux (the total amount of power the irLED can provide) is proportional to the Half Angle (distribution angle of power) times the Radiant Intensity (concentration of power or power density in an area). However, the irLED current ultimately determines if the max amount of radiant flux is provided. To understand all these concepts better, consider the analogy of a 100 watt light bulb. The 100 watt rating is the analogous to the radiant flux. Since a light bulb radiates in all directions, the half angle is fairly large. In order to reduce the half angle and increase the radiant intensity, a parabolic reflector could be placed around the light bulb to direct the path of the light. Although the light bulb is rated at 100 watts, it is the amount of current passing through it that determines the power emitted. Therefore, a bulb that is rated at 100 watts does not always provide 100 watts of power. 


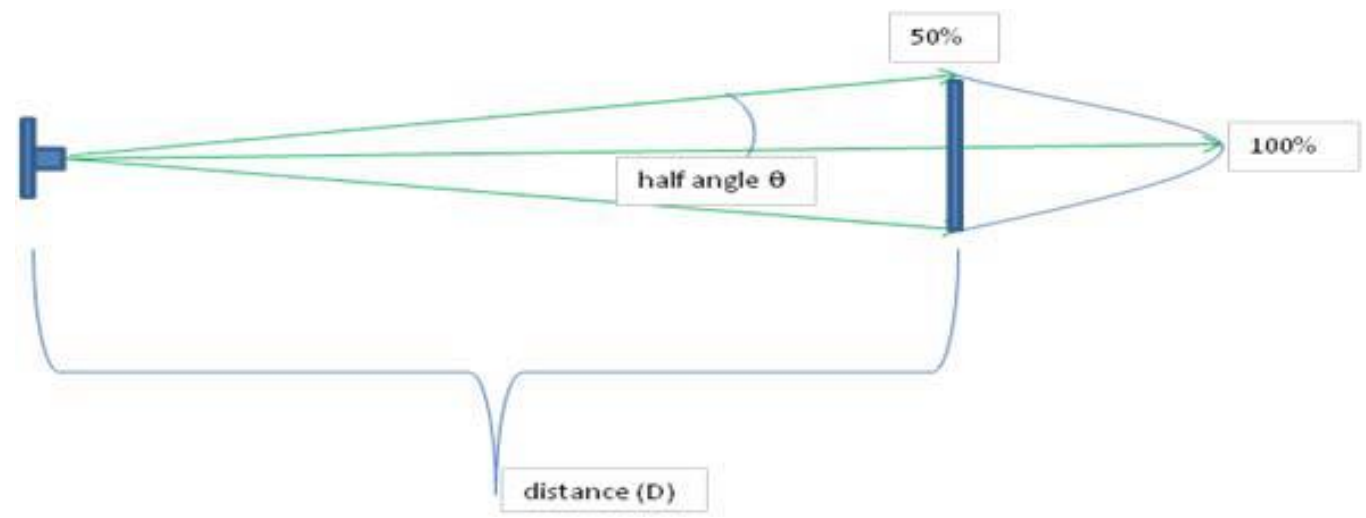

Figure 3. irLED Radiant Intensity vs Half-Angle - The half angle of an infrared LED is the angle in which the radiant intensity is half of the max value or half of the value in the axial direction.

In this thesis, the primary application is to develop a pair of glasses that can detect movement of the eye. Therefore, the design of the glasses calls for placing the sensor and the irLEDs around the rim of the glasses as illustrated in Figure 4.

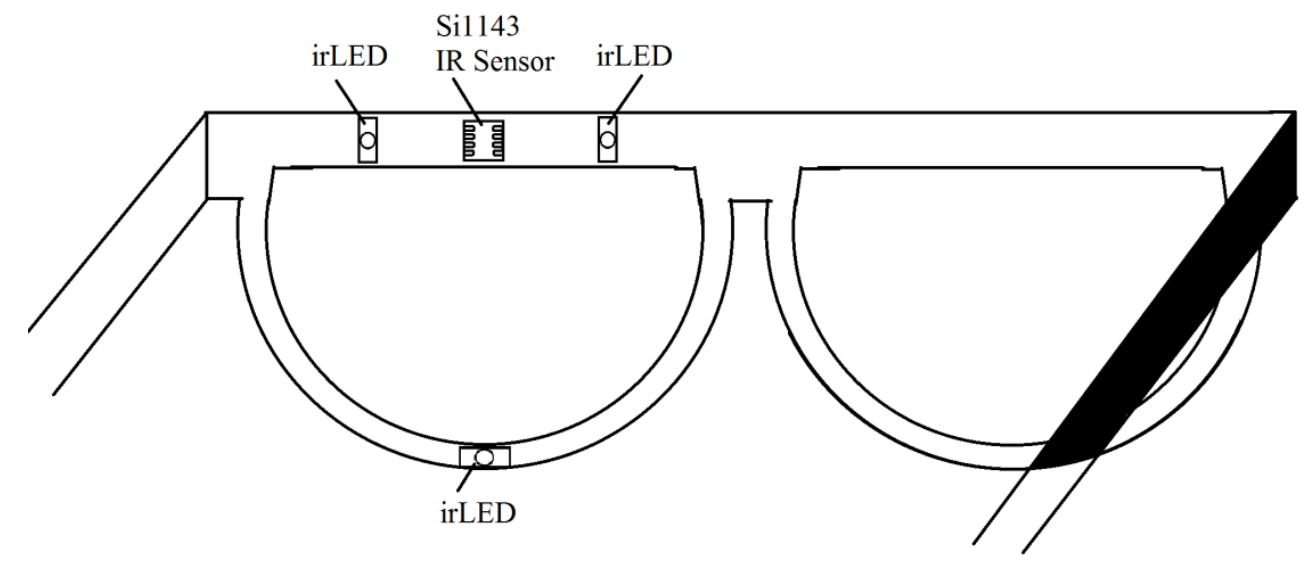

Figure 4. Design of Concept Glasses - This sketch shows the approximate placements of the infrared LEDs and Si1143 proximity/ambient light sensor to track eye movements.

For ideal detection of eye movement, the irLED needs a half angle that covers a good portion of the eye. Given the distance from the rim to the eye and the height from 
the rim of the glasses to the center of the lenses (or center of the eyes), the half angle can be calculated using basic trigonometry as per Equation (1).

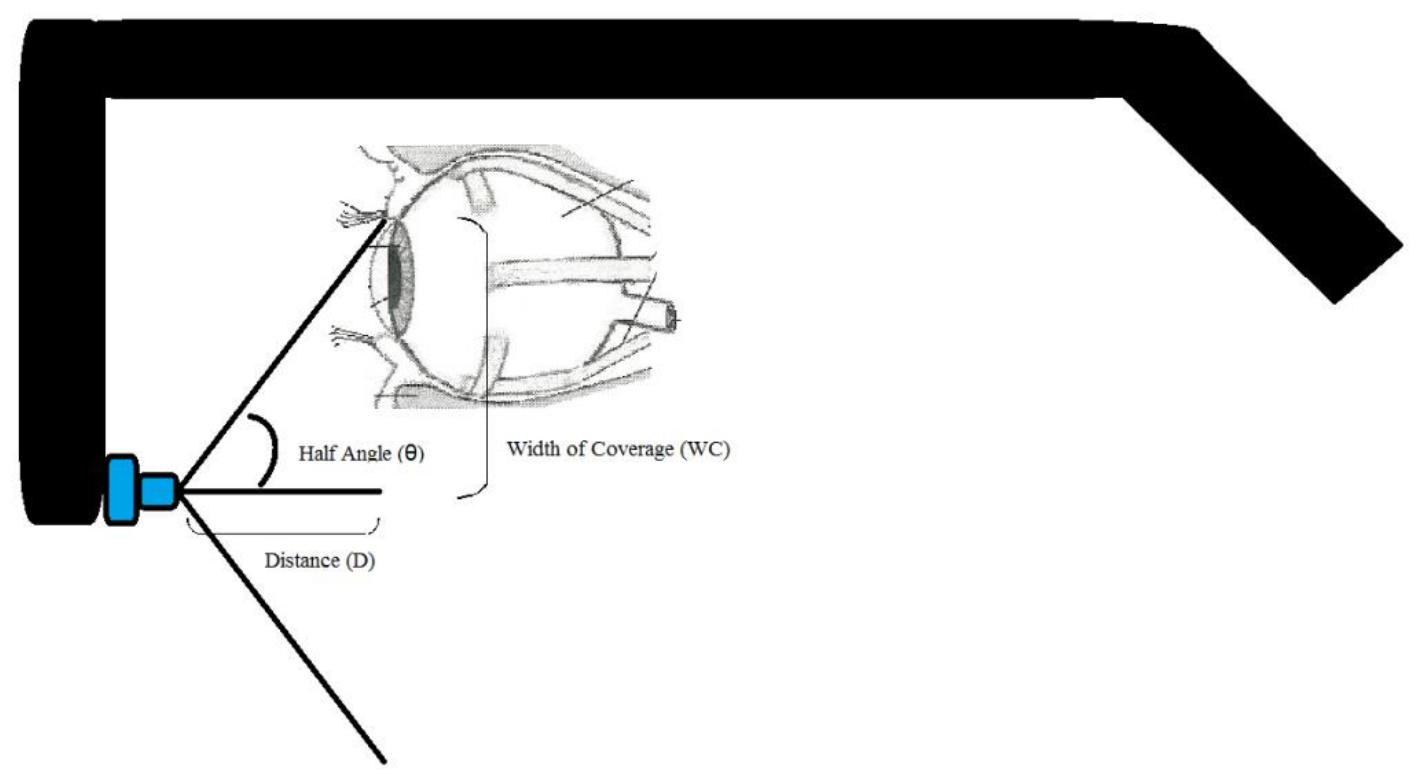

Figure 5. Side View of Concept Glasses - This sketch shows how the infrared LED will shine light on the eye. For ideal use of the infrared LED, the half angle should cover the entire eye.

$$
\theta=\tan ^{-1}(W C / D)
$$

With approximate values of $1 \mathrm{~cm}$ for the distance and $1.5 \mathrm{~cm}$ for the Width of Coverage, the Half Angle $\theta$ would be calculated to be around $56^{\circ}$.

Another important consideration in choosing the irLED's radiant intensity is to know the minimum irradiance level needed at the sensor. Table 2 from Silicon Laboratories Application Note 521 [10] shows typical irradiance $\mathrm{E}_{\mathrm{e}}$ design target values. 


\begin{tabular}{|c|c|c|c|c|}
\hline $\begin{array}{l}\text { Ee (sensor) } \\
\text { Minimum }\end{array}$ & HSIG Bit & $\begin{array}{l}\text { IR Photo } \\
\text { Diode }\end{array}$ & $\begin{array}{c}\text { ADC } \\
\text { Integration } \\
\text { Time }\end{array}$ & Usage (no overlay assumed) \\
\hline $0.25 \mathrm{uW} / \mathrm{cm}^{2}$ & No & Large & $408.6 \mu \mathrm{s}$ & Artificial Lighting CFL $<63$ Ix; Incandescent $<23$ Ix \\
\hline $0.5 \mathrm{uW} / \mathrm{cm}^{2}$ & No & Large & $204.8 \mu \mathrm{s}$ & Artificial Lighting CFL $<125 \mathrm{Ix}$; Incandescent $<56 \mathrm{Ix}$ \\
\hline $1 \mathrm{uW} / \mathrm{cm}^{2}$ & No & Large & $102.4 \mu \mathrm{s}$ & Artificial Lighting CFL $<250$ Ix; Incandescent $<112$ Ix \\
\hline $2 \mathrm{uW} / \mathrm{cm}^{2}$ & No & Large & $51.2 \mu \mathrm{s}$ & Artificial Lighting CFL $<500$ Ix; Incandescent $<225$ Ix \\
\hline $4 \mathrm{uW} / \mathrm{cm}^{2}$ & No & Large & $25.6 \mu \mathrm{s}$ & Artificial Lighting CFL $<1 \mathrm{klx}$; Incandescent $<450 \mathrm{~lx}$ \\
\hline $8 \mathrm{uW} / \mathrm{cm}^{2}$ & Yes & Large & $25.6 \mu \mathrm{s}$ & Artificial Lighting CFL $<2$ klx; Incandescent $<900 \mathrm{~lx}$ \\
\hline $16 \mathrm{uW} / \mathrm{cm}^{2}$ & Yes & Large & $25.6 \mu \mathrm{s}$ & Artificial Lighting CFL $<4 \mathrm{klx}$; Incandescent $<1.8 \mathrm{klx}$ \\
\hline $32 \mathrm{uW} / \mathrm{cm}^{2}$ & Yes & Large & $25.6 \mu \mathrm{s}$ & Artificial Lighting CFL $<8 \mathrm{klx}$; Incandescent $<3.6 \mathrm{klx}$ \\
\hline $58 \mathrm{uW} / \mathrm{cm}^{2}$ & Yes & Large & $25.6 \mu \mathrm{s}$ & Artificial Lighting CFL $<14.5 \mathrm{klx}$; Incandescent $<6.5 \mathrm{klx}$ \\
\hline $7 \mathrm{uW} / \mathrm{cm}^{2}$ & Yes & Large & $25.6 \mu \mathrm{s}$ & Indirect Sunlight $<16 \mathrm{klx}$ \\
\hline $22 \mathrm{uW} / \mathrm{cm}^{2}$ & Yes & Small & $51.2 \mu \mathrm{s}$ & Direct Sunlight $<80 \mathrm{klx}$ \\
\hline $45 \mathrm{uW} / \mathrm{cm}^{2}$ & Yes & Small & $25.6 \mu \mathrm{s}$ & Direct Sunlight $<190 \mathrm{klx}$ \\
\hline
\end{tabular}

Table 2. Typical Irradiance Design Targets [10] - These minimum irradiance values at the sensor are based on current illumination conditions, the ADC integration time, the IR photo diode used, and the HSIG bit setting.

In general, $8 \mu \mathrm{W} / \mathrm{cm}^{2}$ is a good minimum irradiance target to use for indoor applications and operation outdoors. In order to calculate the minimum Radiant Intensity requirement for the irLED with this irradiance value at the sensor, the surface of the eye is assumed to be a Lambertian Diffuse surface where the radiation pattern of reflected light forms a cosine radiation pattern. If this holds true, then the irradiance $E_{e}$ at the sensor can be modeled by Equation (2), where $E_{e}$ is the irradiance, $I_{e}(\theta)$ is the radiant intensity, $\rho$ is the reflectivity of the object, $A$ is the area of the object, and D is the distance from the mid-point between the sensor and the irLED.

$$
E_{e}=\frac{I_{e}(\theta)(\cos \theta)^{2} \rho A}{\pi D^{4}}
$$


By rearranging the equation, the radiant intensity $I_{e}$ can be solved for.

Unfortunately, it is difficult to find a value for the reflectivity of the eye and renders the equation impossible to solve. Since the OSRAM SFH 4056 is one of the few irLEDs that is small in size and can handle the Si1143's max current drive setting, this irLED was chosen.

\subsubsection{Safety Concerns}

The issue of safety standards arises whenever optical radiation is associated with sensitive parts of the body such as the eye. Infrared radiation causes damages when the irradiated tissue overheats and results in the destruction of the cells. Small concentrations of infrared light are known to be safe for the human eye. The concern comes from determining the limit of this exposure. Since infrared is not visible to the human eye, aversion responses such as blinking or iris contraction would not normally occur. As such, the lamp safety standard IEC-62471 [11] outlines the hazard exposure limits for most infrared LED applications. The maximum allowed irradiance $E_{I R}$ of the cornea for different time scales of exposure are defined in Equations (3) and (4), where $E_{\lambda}$ is the spectral radiance in $\mathrm{W} \cdot \mathrm{m}^{-2} \cdot \mathrm{nm}^{-1}, \Delta \lambda$ is the bandwidth in $\mathrm{nm}$, and $\mathrm{t}$ is exposure time in seconds. The irradiance $\mathrm{E}_{\mathrm{e}}$ can be calculated from the radiant intensity $\mathrm{I}_{\mathrm{e}}$ and the distance d using the inverse square law as shown in Equation (5). With pulsed LEDs, the time averaged values of irradiance is used in the exposure limit. Equation (6) shows how to calculate this value.

$$
E_{I R}=\sum_{\lambda=780}^{3000} E_{\lambda} \cdot \Delta \lambda \leq 18000 \cdot t^{-0.75} \quad\left[W \cdot m^{-2}\right], \quad \text { for } t \leq 1000 s
$$




$$
\begin{gathered}
E_{I R}=\sum_{\lambda=780}^{3000} E_{\lambda} \cdot \Delta \lambda \leq 100 \quad\left[W \cdot \mathrm{m}^{-2}\right], \quad \text { for } t>1000 \mathrm{~s} \\
E_{e}=I_{e} / d^{2} \\
E_{e, \text { time avg }}=E_{e, p u l s e} \cdot D=E_{e, p u l s e} \cdot t_{\text {pulse }} / t_{\text {period }}
\end{gathered}
$$

Likewise, the maximum allowed burn hazard weighted radiance $\mathrm{L}_{\mathrm{IR}}$ of the retina for different time scales of exposure are defined in Equations (7) and (8), where $\mathrm{L}_{\lambda}$ is the spectral radiance in $\mathrm{W} \cdot \mathrm{m}^{-2} \cdot \mathrm{sr}^{-1} \cdot \mathrm{nm}^{-1}, \mathrm{R}(\lambda)$ is the burn hazard weighting function, $\Delta \lambda$ is the bandwidth in nanometers, $t$ is the exposure time in seconds, and $\alpha$ is the angular subtense in radians. Equations (9) and (10) calculate the burn hazard function and the angular subtense. According to OSRAM Application Note on Eye Safety of IREDs [14], a good approximation for $\mathrm{L}_{\mathrm{IR}}$ is given in Equation (11), where 1 and $\mathrm{w}$ are the length and width respectively of the active area of the light source.

$$
\begin{gathered}
L_{R}=\sum_{\lambda=380}^{1400} L_{\lambda} \cdot R(\lambda) \cdot \Delta \lambda \leq \frac{50000}{\alpha \cdot t^{0.25}}\left[\mathrm{~W} \cdot \mathrm{m}^{-2} \cdot \mathrm{sr} r^{-1}\right], \text { for } 10 \mu s \leq t \\
\leq 10 \mathrm{~s} \\
L_{I R}=\sum_{\lambda=780}^{1400} L_{\lambda} \cdot R(\lambda) \cdot \Delta \lambda \leq \frac{6000}{\alpha}\left[\mathrm{W} \cdot \mathrm{m}^{-2} \cdot s r^{-1}\right], \text { for } t>10 \mathrm{~s} \\
R(\lambda)=10[(700-\lambda) / 500] \\
\alpha=(l+w) / 2 d
\end{gathered}
$$




$$
L_{I R} \approx \frac{I_{e} \cdot R(\lambda)}{((l+w) / 2)^{2}}
$$

For the OSRAM SFH 4056, the typical max radiant intensity value in the axial direction is $225 \mathrm{~mW} / \mathrm{sr}$. Assuming that the user wears the glasses for an extended period of time, Equation (4) will be used to calculate the exposure limit. Another assumption takes into account that the irLEDs will not shining directly into the eye but at an angle of $56^{\circ}$ as calculated from Equation (1). The values in that example can also be used to calculate the hypotenuse or distance $\mathrm{d}$ which turns out to be $1.8 \mathrm{~cm}$.

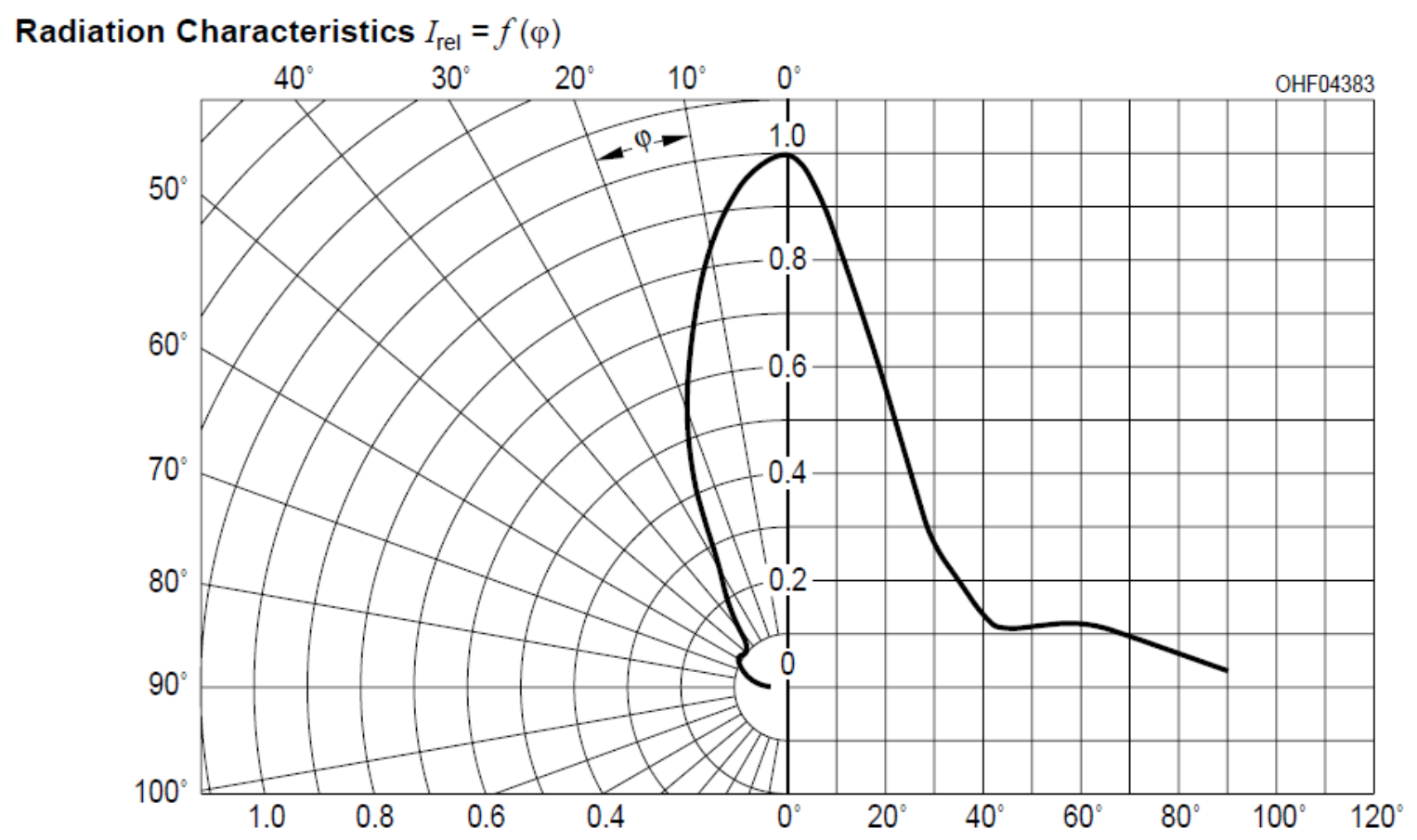

Figure 6. OSRAM SFH 4056 Radiation Characteristics [13] - The radiant intensity decreases greatly as the angle increases. The half angle is shown to be at $22^{\circ}$.

From Figure 6, this angle gives a relative radiant intensity value of around 0.11 .

Lastly, since the three LEDs are pulsed, the values of the pulse time and time period are given as $25.6 \mu \mathrm{s}$ and $3.27 \mathrm{~ms}$ respectively from Section 3.3. With all these values, the 
cornea exposure limit for OSRAM SFH 4056 LED is calculated in Equations (12), (13), and (14).

$$
\begin{gathered}
I_{e, r e l}=0.11 \cdot I_{e, t y p}=0.11 \cdot 225 \frac{\mathrm{mW}}{\mathrm{sr}}=24.75 \frac{\mathrm{mW}}{\mathrm{sr}}=0.02475 \frac{\mathrm{W}}{\mathrm{sr}} \\
E_{e, p u l s e}={ }^{I_{e, r e l}} \mathrm{~d}^{2}=\frac{0.02475}{0.018^{2}}=76.39 \frac{\mathrm{W}}{\mathrm{m}^{2}} \\
E_{e, t i m e ~ a v g}=76.39 \frac{\mathrm{W}}{\mathrm{m}^{2}} \cdot \frac{3 \times 25.6 \mu \mathrm{s}}{3.27 \mathrm{~ms}}=1.794 \frac{\mathrm{W}}{\mathrm{m}^{2}} \leq 100 \frac{\mathrm{W}}{\mathrm{m}^{2}}
\end{gathered}
$$

The results of Equation (14) show that the spectral radiance is well below the cornea exposure limit given these values. The value for the retinal exposure limit must also be calculated for when the exposure time is great. Given that the length and width of the active chip area are $0.2 \mathrm{~mm}$ and $0.2 \mathrm{~mm}$ respectively, the approximation Equation (16) is used for $\lambda=850 \mathrm{~nm}$.

$$
\begin{gathered}
\alpha=0.0002 / .018=0.011 \\
L_{I R} \approx \frac{\left(I_{e, r e l} \cdot 10^{(700-\lambda) / 500]}\right)}{\left(\frac{(l+w)}{2}\right)^{2}}=\frac{0.02475 \cdot 10^{-0.3}}{0.0002^{2}}=310109.6 \leq \frac{6000}{0.011} \\
=540000 \frac{W}{m^{2} \cdot s r}
\end{gathered}
$$

The results of Equation (16) show that the spectral radiance for retina exposure is below the limit as well. Therefore, this analysis concludes that the OSRAM SFH 4056 Infrared LED would not emit enough harmful radiation given these specifications. 


\subsubsection{Power Consumption of OSRAM SFH 4056 LED}

According to the datasheet [13], the max power consumed by SFH 4056 occurs when the Si1143 drives the LED at its highest current level of $359 \mathrm{~mA}$. Therefore, the duty cycle from Equation (14) of the LED pulse is used to calculate the average power consumed according to Equation (17). The forward voltage of the SFH 4056 is also rated at $1.6 \mathrm{~V}$.

$$
P_{\text {avg }}=I_{\text {inst }} V_{\text {inst }} \cdot D=359 \mathrm{~mA} \cdot 1.6 \mathrm{~V} \cdot \frac{25.6 \mu \mathrm{s}}{3.27 \mathrm{~ms}}=4.5 \mathrm{~mW}
$$

The value of $4.5 \mathrm{~mW}$ would be the max possible power draw for a single LED. If all three LEDs on the circuit are driven to the max, a max possible value of $13.5 \mathrm{~mW}$ would occur. Further testing and analysis shown in Section 4.8 would prove that this max value of current drive is not needed to detect eye movement and that the power consumed in the experiment is much less than the theoretical value. In addition to these facts, it is stated that the retina reflects different amounts of infrared light at different frequencies [3]. At $850 \mathrm{~nm}$, the retina reflects $90 \%$ of incident light whereas at $950 \mathrm{~nm}$ the retina reflects $40 \%$. With most of the radiant intensity concentrated at $860 \mathrm{~nm}$ for the $\mathrm{SFH} 4056$ as shown in Figure 7, this further proves that only a small percentage of a small amount of power is being absorbed by the eye. 


\section{Relative Spectral Emission}

$$
\mathrm{I}_{\text {rel }}=f(\lambda)
$$

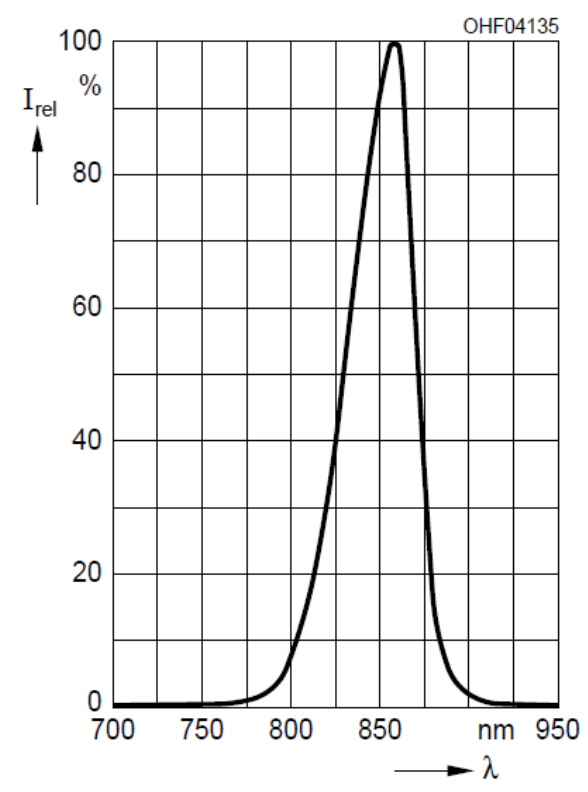

Figure 7. OSRAM SFH 4056 Relative Spectral Emission [13] - The graph displays the radiant intensity emitted across the spectrum of wavelengths. The wavelength at peak emission is shown as $860 \mathrm{~nm}$.

\subsection{Preliminary Testing}

This section shows the initial testing of the Si1143 sensor using the Si1143

Development Board shown in Figure 8. The results of the initial testing will help prove whether or not the Si1143 sensor is sensitive enough to detect movement of the eye. The testing will also characterize the performance of the system. 


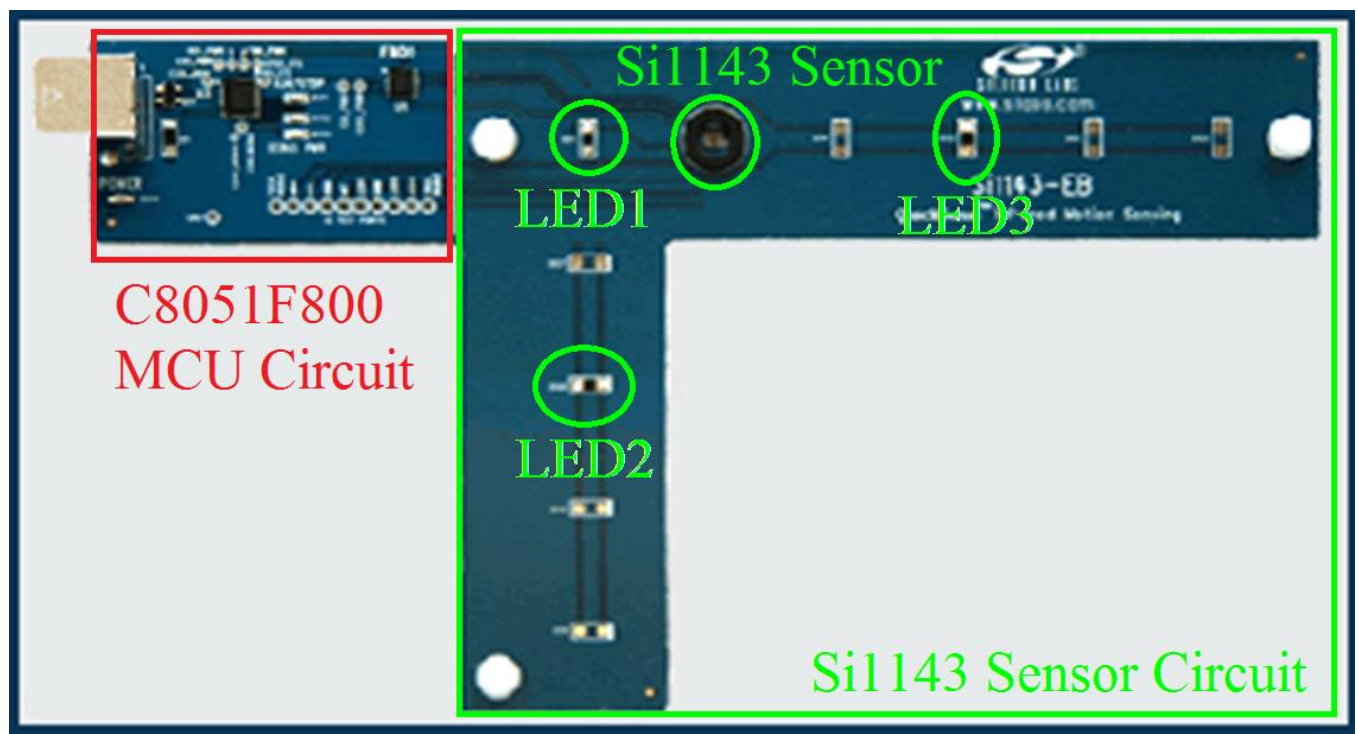

Figure 8. Si1143 Development Board - The board features three OSRAM SFH 4056 Infrared LEDs, the Si1143 sensor, and the C8051F800 microcontroller.

The Si1143 Development Board can be divided into two regions. The first region highlighted in red on Figure 8 consists of the C8051F800 microcontroller circuit. This region is where the 8051 communicates as the host to the sensor. The second region highlighted in green represents the Si1143 sensor circuit. The sensor and each LED in the figure are also identified.

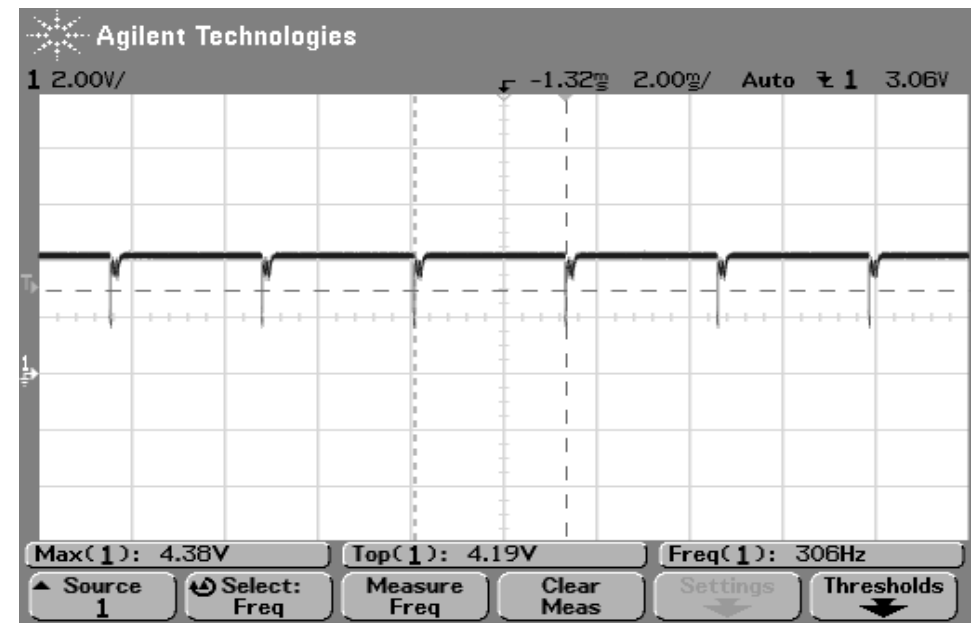

Figure 9. Measurement Time between each LED pulse - The time period between each LED pulse measures $3.27 \mathrm{~ms}$. The speed at which the Si1143 measures data is $306 \mathrm{~Hz}$. 
Testing was done to verify the specifications on the Si1143 datasheet. Figure 9 shows the period in which LED1 was pulsed. The frequency was measured to be $306 \mathrm{~Hz}$ which is equivalent to a period of about $3.27 \mathrm{~ms}$.

\begin{tabular}{|c|c|c|c|c|c|}
\hline Actively Measuring Time ${ }^{4}$ & $\begin{array}{c}\text { Single PS } \\
\text { ALS VIS + ALS IR } \\
\text { Two ALS plus three PS }\end{array}$ & $\begin{array}{l}- \\
- \\
-\end{array}$ & $\begin{array}{l}155 \\
285 \\
660\end{array}$ & $\begin{array}{l}- \\
- \\
-\end{array}$ & $\begin{array}{l}\mu \mathrm{s} \\
\mu \mathrm{s} \\
\mu \mathrm{s}\end{array}$ \\
\hline
\end{tabular}

Table 3. Actively Measuring Time Performance Characteristic [9] - These are typical measuring times for each type of measurement taken by the Si1143. As more parameters are measured, the measuring time increases.

Typical values for measuring times are shown in Table 3. After the LED is pulsed, the sensor takes 155 microseconds to make a single Proximity Sensing (PS) measurement and measure the light that has just been reflected. If adding the additional Ambient Light Source for Visible Light (ALS VIS) and Ambient Light Source for Infrared Light (ALS IR) to three PS measurements, the sensor takes 660 microseconds to make the measurement which is well in the time span before the next pulse cycle.

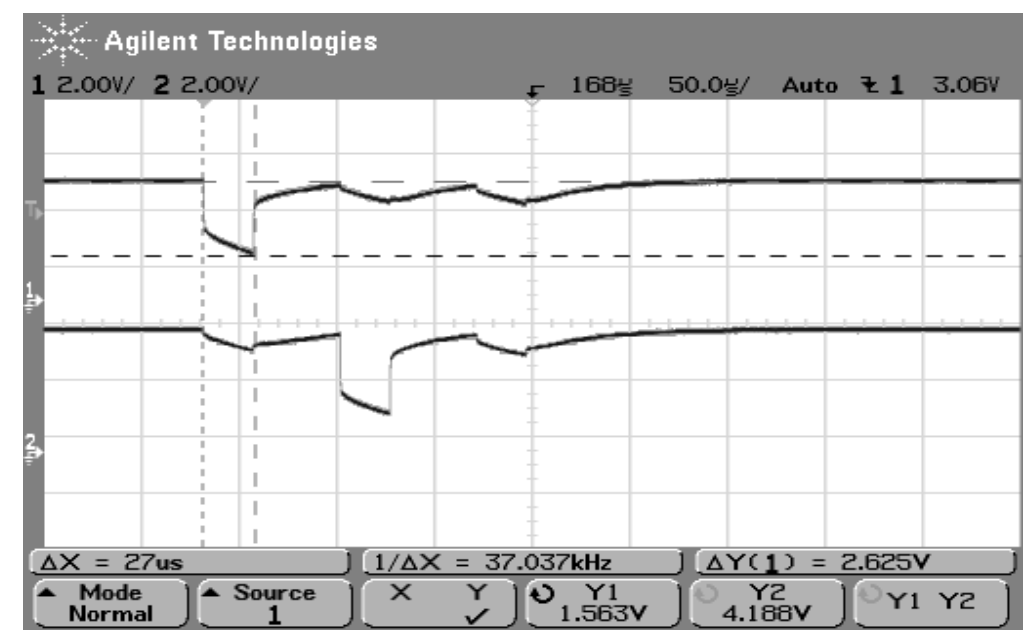

Figure 10. Measurements of LED1 and LED2 Pulse Width - Channel 1 measures the voltage drop across LED1 while Channel 2 measures the voltage drop across LED2. The waveform shows a measured pulse width of 27 microseconds. 
Figure 10 shows the result of manually measuring the pulse width durations of LED1 on channel 1 and LED2 on channel 2. A measured value of 27 microseconds falls well within the typical values of 25.6 - 30 microseconds as shown in Table 4. As channel 1 is driving LED1, a noticeable voltage drop appears on channel 2 of LED2 (and LED3 if it was measured). This occurrence is typical as each LED driver would create a voltage drop across its respective LED and affect nearby voltage levels of non-driven LEDs. The time between the two pulse widths in channel 1 and channel 2 would also be where a PS measurement would be made.

\begin{tabular}{|l|c|l|l|l|l|l|}
\hline $\begin{array}{l}\text { LED1, LED2, LED3 } \\
\text { Pulse Width }\end{array}$ & $t_{P S}$ & & - & 25.6 & 30 & $\mu \mathrm{s}$ \\
\hline
\end{tabular}

Table 4. LED Pulse Width Performance Characteristics [9] - The typical and max time for each LED pulse width are shown to be 25.6 microseconds and 30 microseconds respectively.

The development board also came with a Performance Analysis Software Tool to quickly operate the board. With a simple plug in to the USB port and a quick installation of the software, preliminary measurements were taken and shown in Figure 11 below. Figure 11 shows the result of placing a subject's left eye within one inch away from the Sil143 sensor on the development board and glancing to the left. 


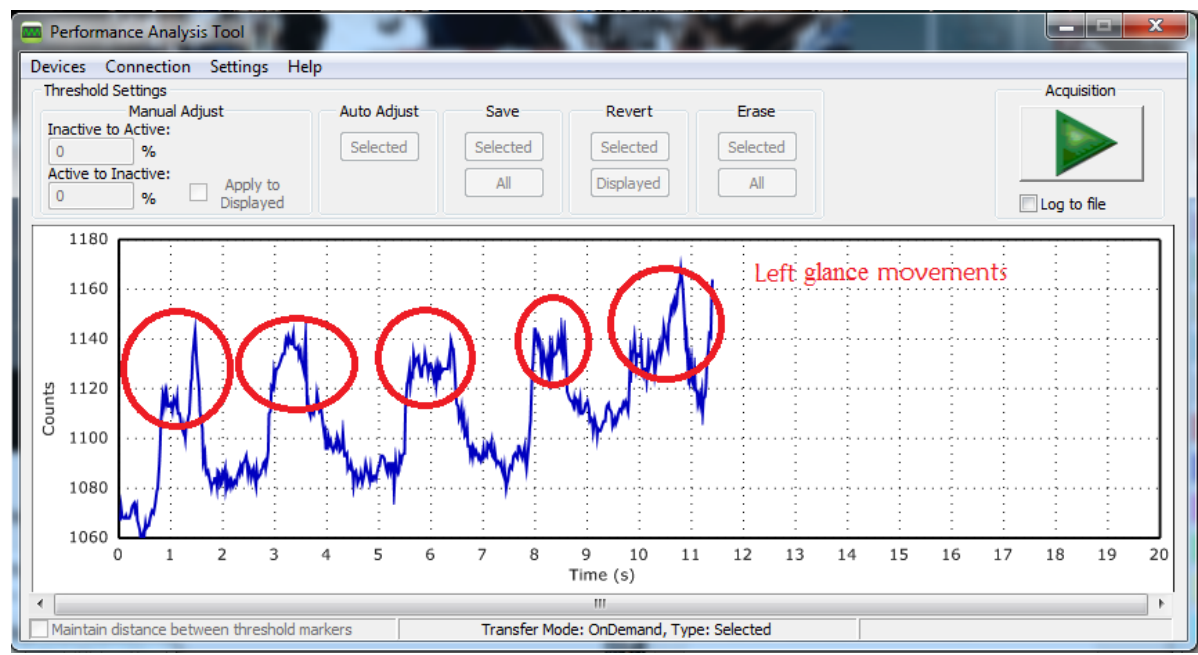

Figure 11. Left Glance Detection of the Left Eye - As the left eye makes left glances, the counts on the graph increase and then decrease. The graph shows five of these left glances.

The waveform showed a noticeable variance in the counts read from the $1^{\text {st }}$

Proximity Sensing channel (PS1) when the left eye looked to the left. Five left glances were made with the left eye and five corresponding peaks were observed. The preliminary test concluded that sensor was sensitive enough to detect eye movement 


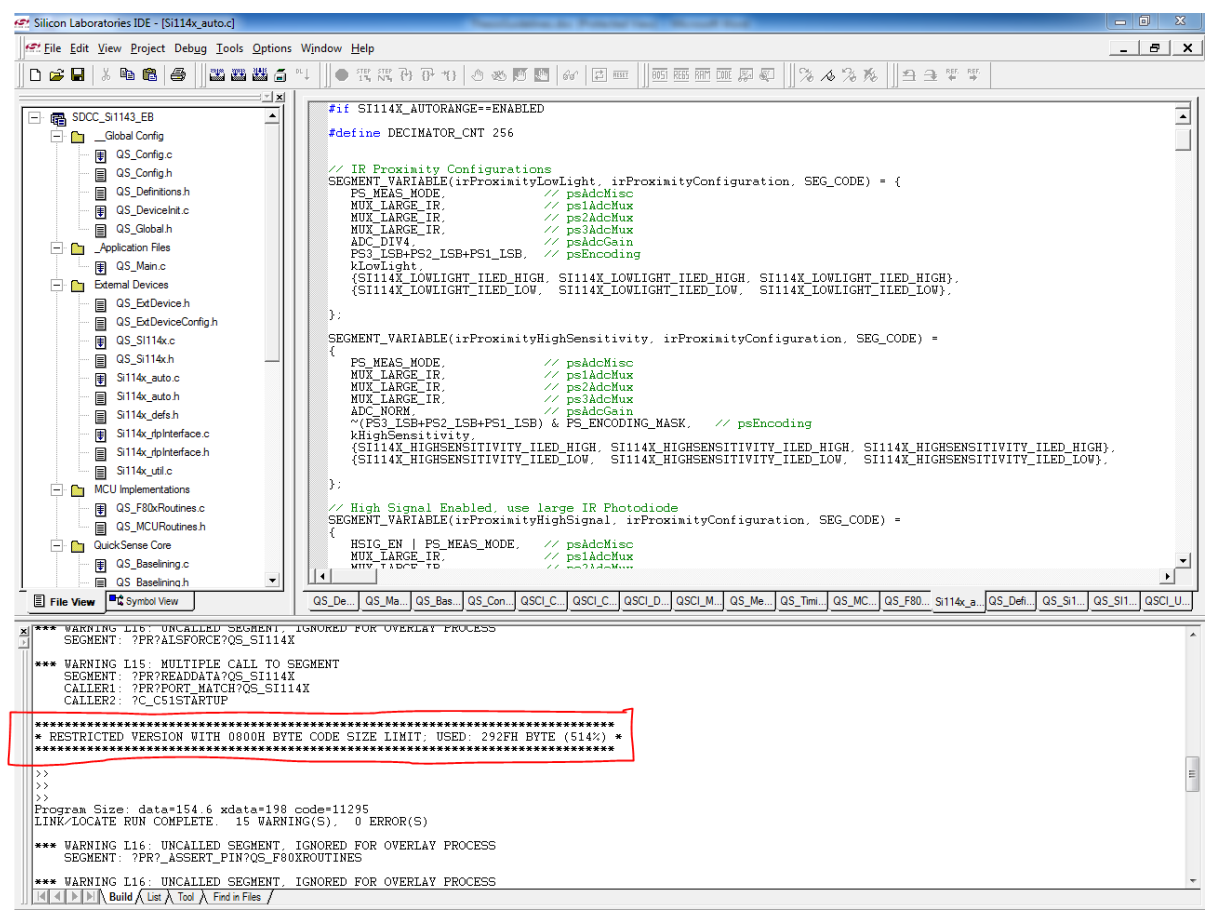

Figure 12. Screenshot of Silicon Laboratories IDE - The highlighted portion in red indicates that the compiled code size limit has been reached.

From this quick and simple proof of concept, the next step involved using the

Silicon Laboratories Integrated Development Environment to program the 8051

microcontroller. Silicon Laboratories released their Quick Sense Application

Programming Interface (API) that includes header files, functions, configuration settings, and definitions for the 8051 microcontroller family and for the Si1143 sensor. However, difficulties arose in using third-party assembler, compiler, and linker for that family of microcontrollers. The third-party CA51 Compiler Kit by Keil had a code size limit restriction on the evaluation version of its compiler. Figure 12 above shows a screenshot of the error encountered when compiling the code. In order to fully compile the code, the CA51 Compiler Kit needed to be purchased. Appendix A shows a quote received from the company. The cost and time required to pursue this route proved too great and an alternative solution was sought to continue development. 


\section{CHAPTER 4. GLASSES PROTOTYPE DESIGN}

This chapter discusses the design of two prototype glasses. The first prototype uses an existing frame with the circuitry attached onto it while the second iteration prototype streamlines the design by creating the entire glasses out of PCB. Each section outlines the design process for each prototype. This design process includes circuit schematic, assembly procedure, software development, results, and analysis.

\subsection{Circuit Schematic and Component Selection}

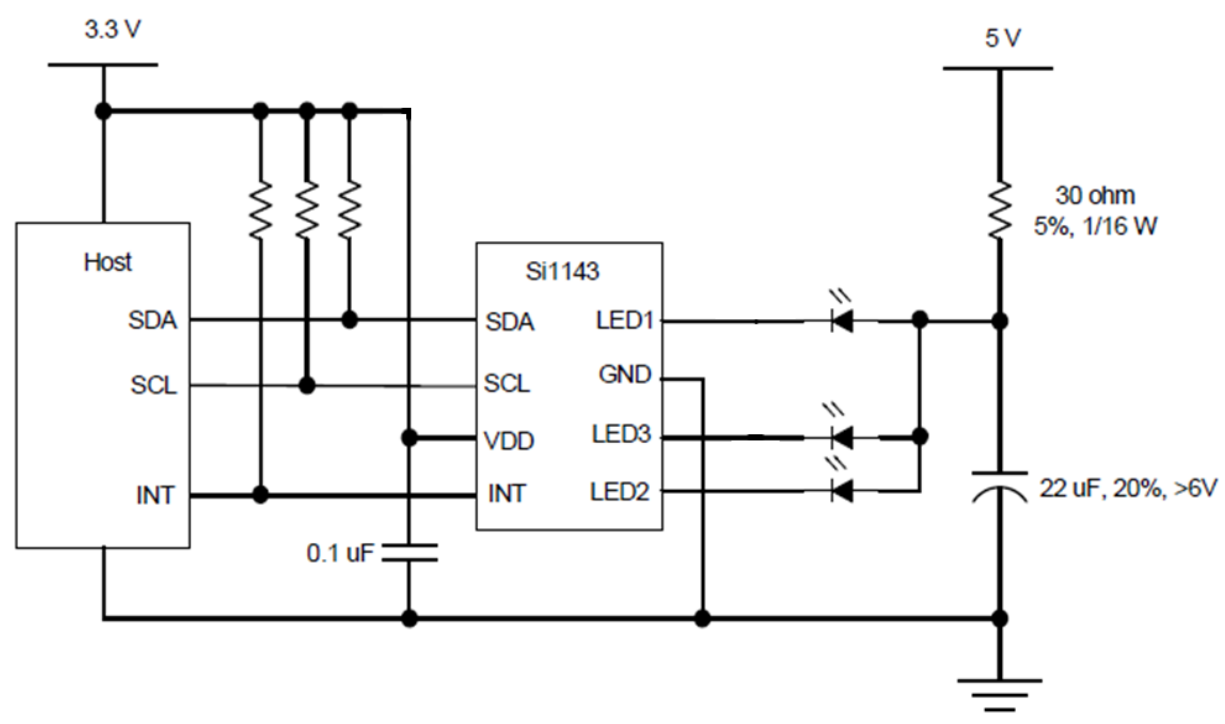

Figure 13. Si1143 Circuit Schematic [12] - A separate voltage rail design was used to ensure enough current could be supplied to the infrared LEDs.

The schematic for the Si1143 sensor shown in Figure 13 was the chosen circuit to implement the sensor for eye tracking. A $0.1 \mu \mathrm{F}$ decoupling capacitor filters out any spurious signal on the supply line. Since the max current draw from the Arduino Uno board is $50 \mathrm{~mA}$ for the $3.3 \mathrm{~V}$ rail, two separate supply rails are used in the design. The 3.3 V line supplies current for all the connected Si1143 devices while the $5 \mathrm{~V}$ line supplies 
current to drive the irLEDs. Three $4.7 \mathrm{k} \Omega$ pull-up resistors are placed on the data lines to insure the logic level stays high when the device is not active. The LED3 pin operates as a factory test pin to the microcontroller and must be pulled up to VDD under boot-up conditions. This pin is effectively pulled to VDD through the $5 \mathrm{~V}$ supply and the forward voltage of $0.5 \mathrm{~V}$ from the irLED when it is not actively driven.

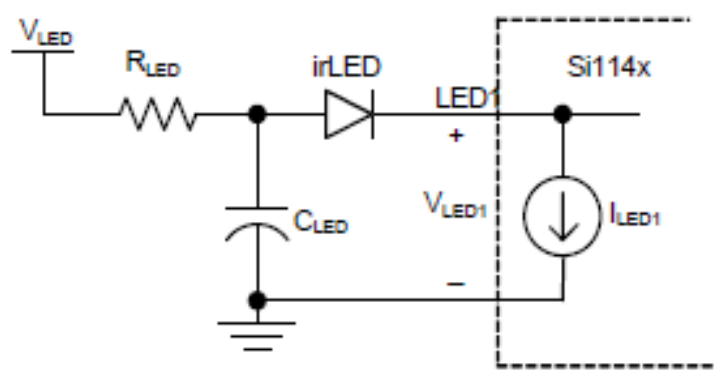

Figure 14. LED Driver Circuit on the Si1143 [12] - The Si1143 controls the amount of current pulsed through the irLED.

The function of the capacitor in Figure 14 is to provide sufficient charge when the irLEDs are pulsed for measurements while the function of the resistor is to charge up the capacitor and minimize instantaneous current draw from the supply rail. The capacitor and resistor values in the LED driver circuit were chosen to optimize circuit performance based on Equation (18) and Equation (19) as outlined in Silicon Lab's Application Note 498 [12].

$$
\begin{aligned}
& C_{\text {LED }}=\frac{\text { Number of LEDs } \times \text { Max ir LED Current } \times 25.6 \mu s \times 2^{(\text {PS_ADC_GAIN })}}{V_{\text {LED }}-V_{F}-0.5} \\
& \mathrm{R}_{\text {LED }}<\frac{\text { Time between Proximity Measurements }}{\mathrm{C}_{\text {LED }} \times 5} \\
& \text { Assuming } \mathrm{C}_{\text {LED }} \text { is greater than or equal to the calculation }
\end{aligned}
$$


With three irLEDs, a max current draw of 359mA, PS_ADC_GAIN set to 0, a $\mathrm{V}_{\mathrm{LED}}$ of $5 \mathrm{~V}$, and a $\mathrm{V}_{\mathrm{F}}$ of $2.2 \mathrm{~V}$ under the max current draw condition, $\mathrm{C}_{\mathrm{LED}}$ is calculated to $12 \mu \mathrm{F}$. To ensure enough capacitance, this value is rounded up to the nearest common capacitance value which is $22 \mu \mathrm{F}$. From Section 3.3, typical times between proximity measurements were around $3.3 \mathrm{~ms}$ which makes resistor values less than or equal to $30 \Omega$ according to Equation (19). 


\subsection{Assembly and Placement Procedure}

\subsubsection{Functionality Verification}

The initial assembly process involved soldering individual ribbon cable wires directly to the sensor to verify functionality. Due to the size of the chip and the solder pads, soldering wires directly to the chip was near impossible. The smallest solder tip size was still too large to touch down on a single pad. Whenever solder connections were made, adjacent connected pads would heat up and break the connection. Even though the wires were eventually soldered on and glued into place for mechanical stability, the setup was not seen as a viable solution to the electrical and mechanical stability of the system.

Through the purchase of breakout boards and assembly service from www.protoadvantage.com, the Si1143 was prototyped onto a breadboard as seen in Figure 15 and Figure 16.

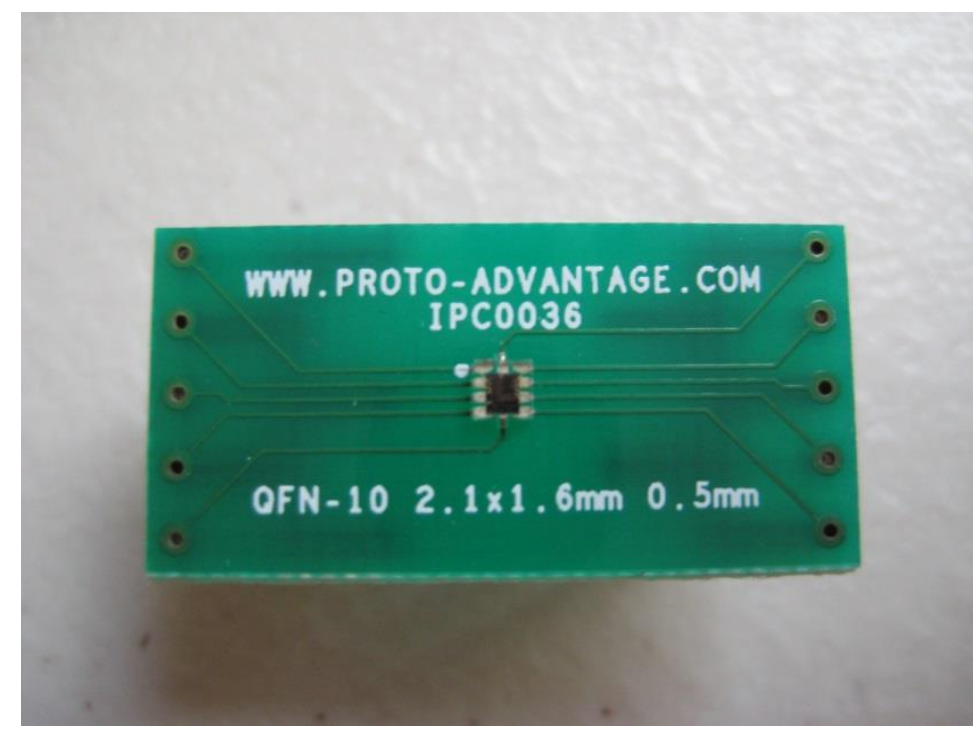

Figure 15. Si1143 Sensor on a QFN-10 Breakout Board - The breakout board enabled access to the pins of the Si1143 sensor for prototype testing. 


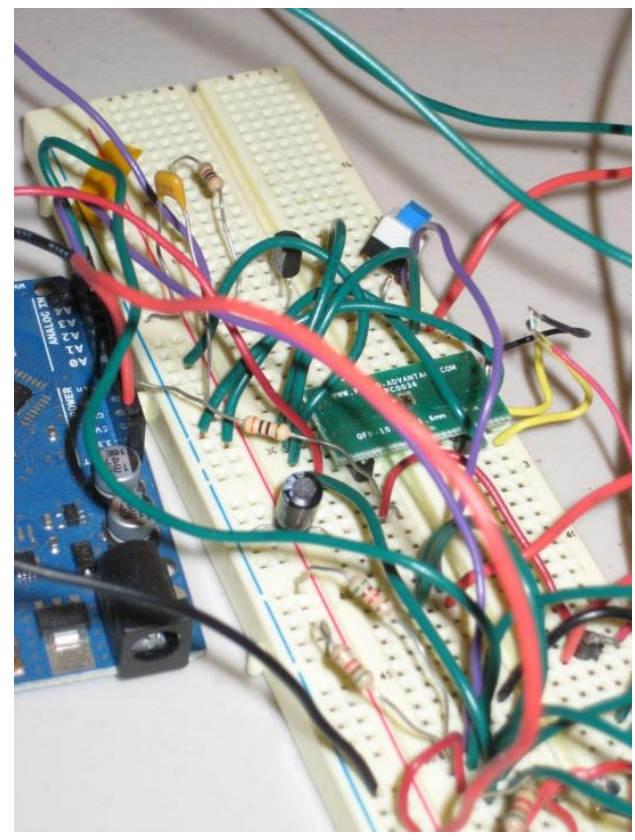

Figure 16. Prototype Design Test Setup on Breadboard - The circuit was first tested on this breadboard setup to verify functionality.

The software interface outlined in the next section verified the functionality of the circuit. Steps were then taken to construct the electronics onto a pair of glasses for the primary application and another test setup to measure heart rate on reflective PPG.

\subsubsection{Glasses Construction}

A pair of Real D 3D glasses shown in Figure 17 was used as the casing and structure for the prototype glasses. The lenses were removed and indentations were cut and filed into the rim of the glasses to allow placement of the LEDs and sensor. 


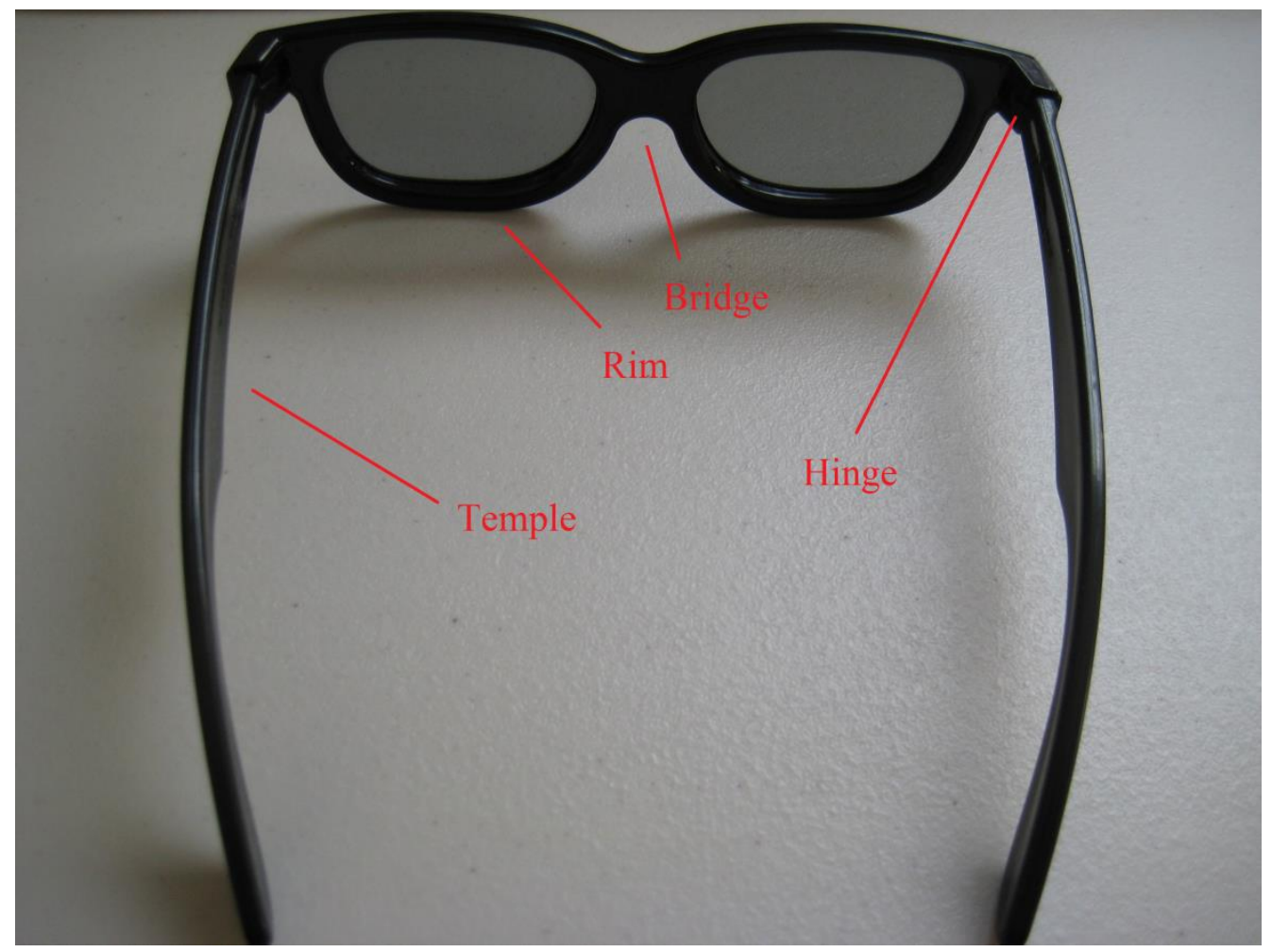

Figure 17. Real D 3D Glasses - These glasses provided the framework for initial prototyping. The names for each part of the glasses are also defined.

In order to discreetly place the PCB breakout board onto the rim of the glasses, a dremel with an attached sanding drum shown in Figure 18 was used to grind the PCB into shape. The PCB was held in place by a vice to allow for steady grinding.

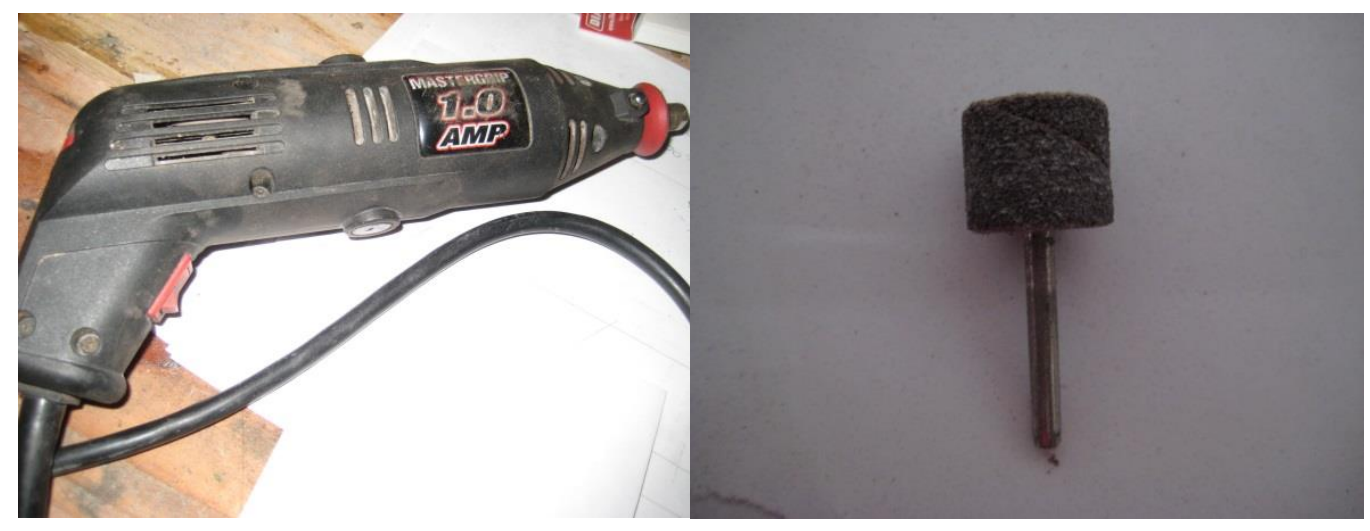

Figure 18. Dremel and Sanding Drum - These tools were used for to cut the breakout board into shape for placement onto the Real D 3D Glasses. 
Because the fiberglass in the FR-4 board turns into dust when sanded away and is dangerous to inhale, a breathing apparatus shown in Figure 19 was donned to ensure proper safety.

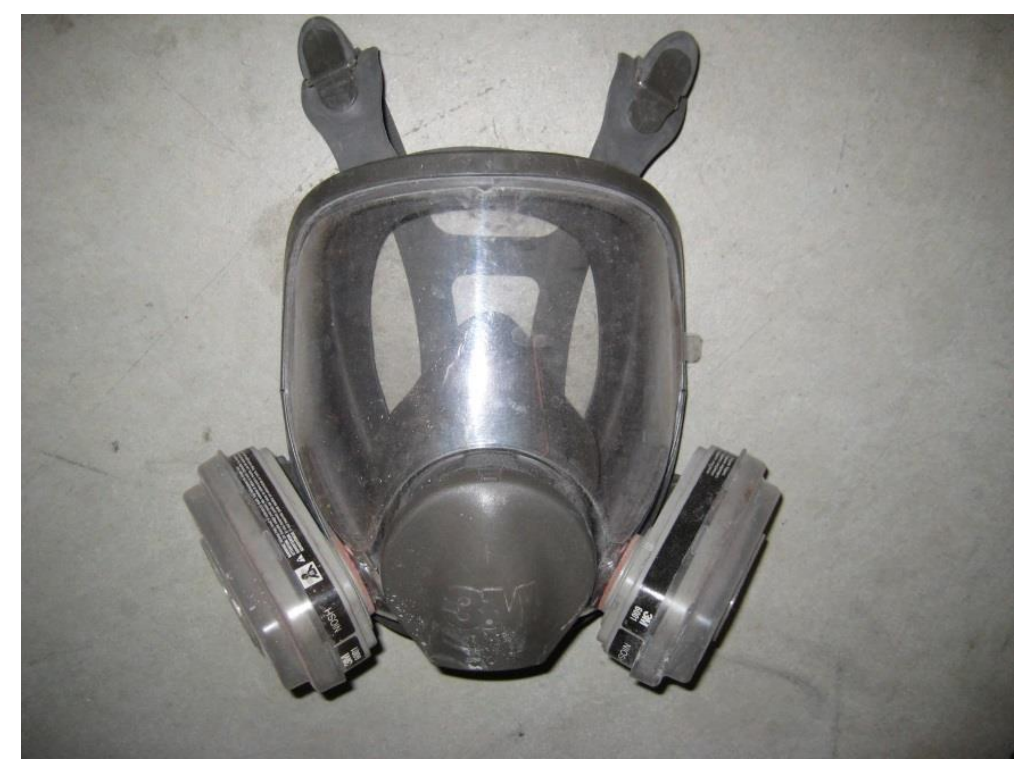

Figure 19. 3M Breathing Apparatus - This mask prevented dangerous FR-4 dust from being inhaled when cuts were made to the PCB board.

Because the PCB was grinded into shape, the pinout holes of the breakout board were inaccessible. This limited access of the pins to the top traces of the PCB. Through careful sanding of the top solder mask layer on the PCB, the copper traces underneath were exposed. Wires were then soldered onto these traces and the electronics were glued in place with a combination of electrical tape, Compton tape, and hot glue. Figure 20 reveals the completed prototype and where each component is located. 


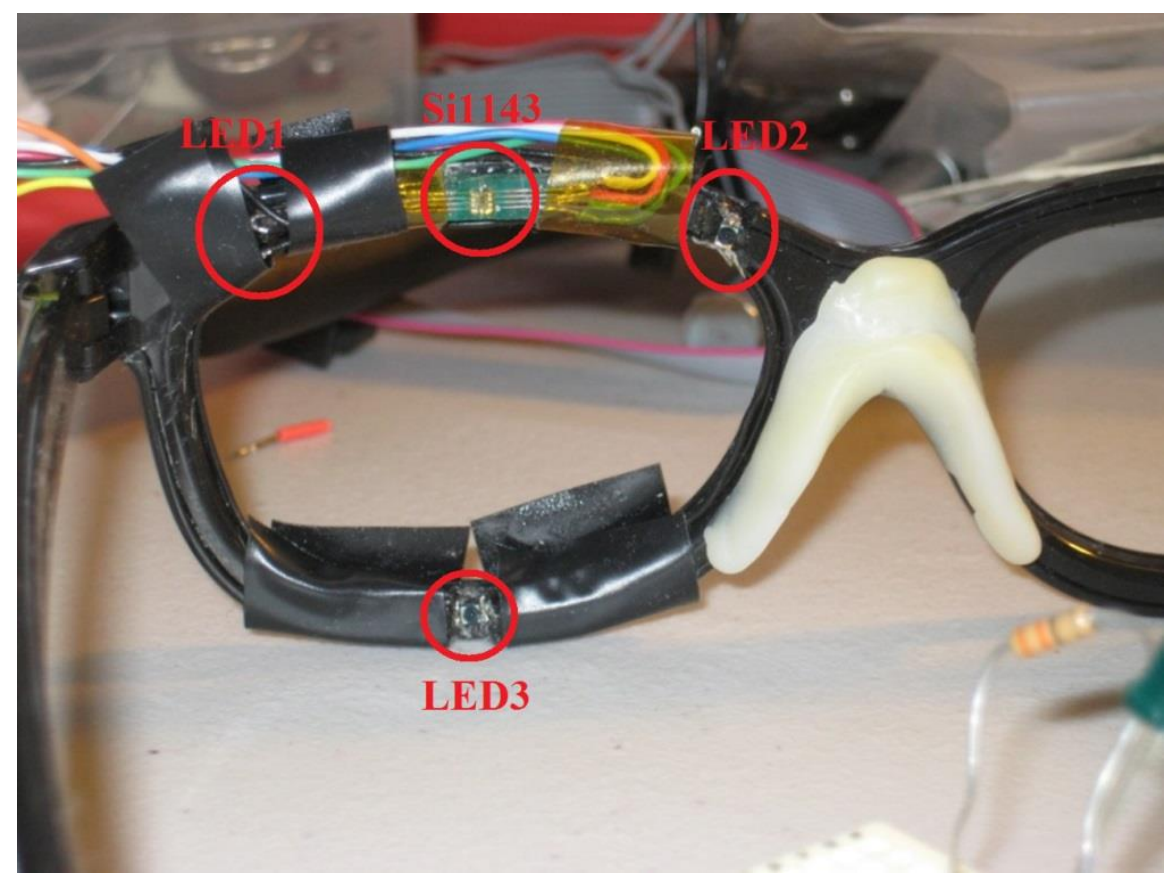

Figure 20. Assembled Glasses Prototype - This prototype was the first design used to test the concept of infrared eye tracking.

Since the Real D 3D glasses by design was placed too close to the face when worn, a moldable plastic nosepiece was made to adjust the distance from the glasses to the face. This moldable plastic was made from a product called Instamorph as shown in Figure 21 below.

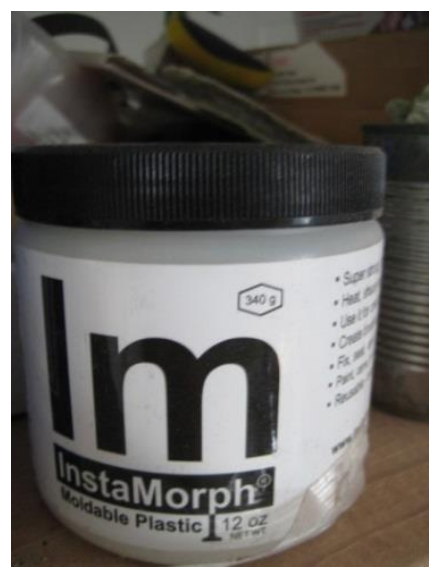

Figure 21. Instamorph Moldable Plastic - This moldable plastic was used to form the nosepiece and earpiece adjustments to the initial and final prototype glasses. 


\subsection{Software and Programming Development}

The first prototype design involved using an Arduino Uno as the microcontroller host and interfacing it with the LabVIEW programming language to do all the programming. In order to enable LabVIEW to talk with the Arduino Uno, the open source LabVIEW Interface for Arduino (LIFA) toolkit, shown in Figure 22, was downloaded from National Instruments' website and loaded onto the Arduino Uno. This firmware sketch would cause the Arduino Uno to act as an $\mathrm{I} / \mathrm{O}$ engine for any commands sent from LabVIEW.

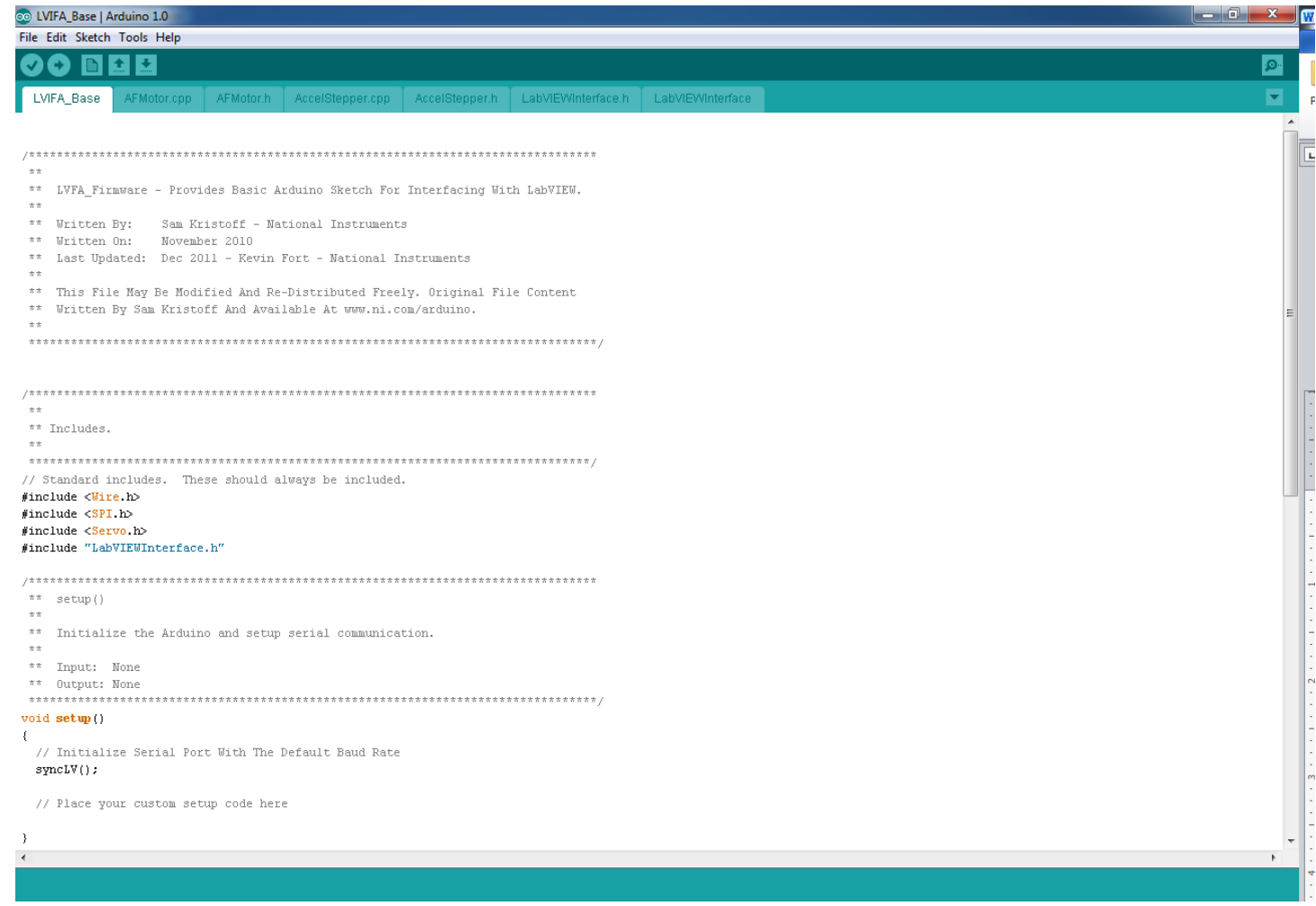

Figure 22. Screenshot of LIFA Firmware Sketch - This sketch enabled LabVIEW to interface with the Arduino.

Pre-programmed Arduino Virtual Instruments (VIs) enable LabVIEW to send data packets to the Arduino which processes these data packets and communicates as the 
host to any sensors or inputs that are connected to it. Then the Arduino relays back the information to LabVIEW via a return data packet.

LIFA does not currently have any interrupt handler functions written in it. As a result, LabVIEW cannot support interrupt handling from the Si1143 sensor. Further work outside the scope of this thesis could be developed to integrate an interrupt handler function in LIFA. Therefore, this thesis ignores the use of any interrupt function in the Si1143 chip when using LIFA.

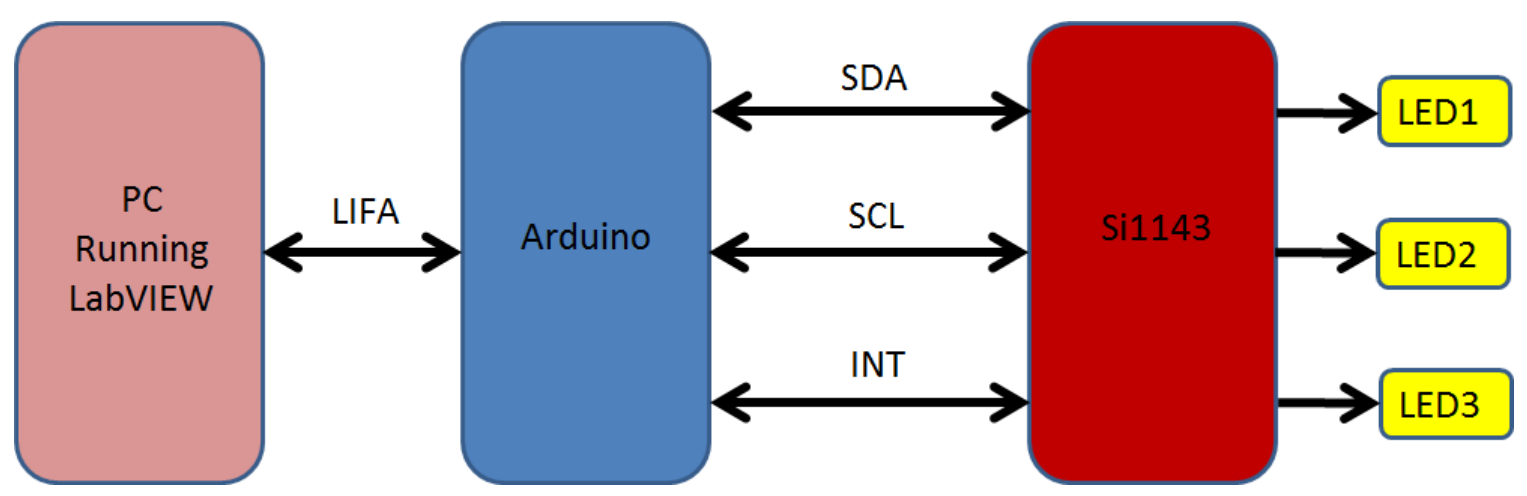

Figure 23. Host Command Structure - This figure illustrates the hierarchal structure of how the computer communicates with the Si1143 sensor.

Through LabVIEW and LIFA, the Arduino communicates to the Si1143 chip using the $\mathrm{I}^{2} \mathrm{C}$ protocol. Information about this protocol can be found in Appendix $\mathrm{C}$ of this thesis. The flowchart describing the code is shown in Figure 24. 


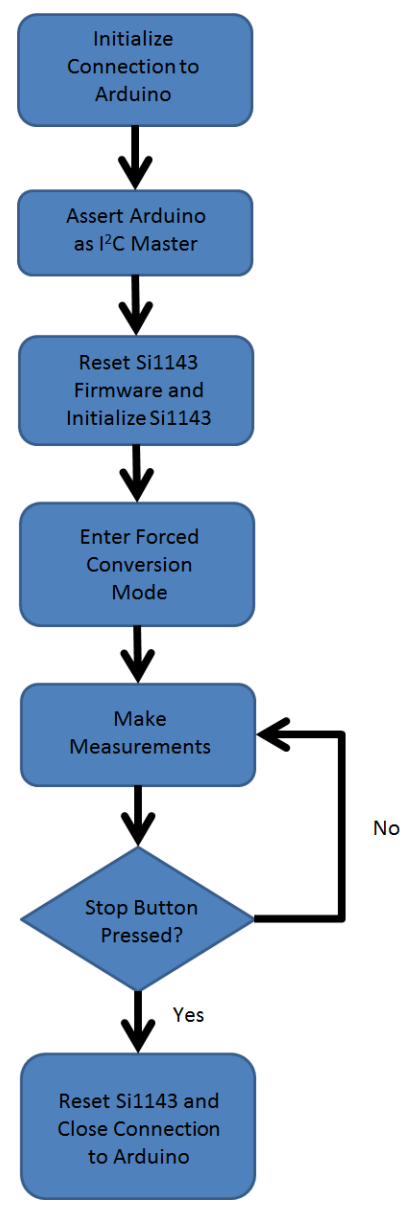

Figure 24. Flowchart of LabVIEW Program - This flowchart outlines the main protocol for measuring and acquiring data from the Si1143 sensor.

First, LabVIEW initializes the connection to the Arduino and sets the Arduino as the master of $\mathrm{I}^{2} \mathrm{C}$ bus. Next, it performs a reset of the firmware on the Si1143 and initializes it by writing the command 0x17 to the HW_KEY register. The program then sets the Si1143 to measure data in Forced Conversion Mode by setting all autonomous measure rates to zero. This action disables the internal sequencer in the sensor. Next, the program enters a continuous loop where it writes a command for the Si1143 to make a measurement and return the data bytes. The data bytes are converted into a numeric count value through Equation (20) and then plotted on a waveform graph. 
During this loop, the user has the option to enable different configuration and current settings and check RAM parameters and register data. Once the stop button is pressed, the program exits the loop, resets the Si1143 chip, and closes the connection to the Arduino.

\subsection{Initial Experimental Results and Analysis}

Figure 25 shows a screenshot of the LabVIEW front panel. The graph on the right of the figure displays a test collection of data from the three Proximity Sensing channels on the Si1143 for eye tracking. Figure 26 shows an actual test where the user is wearing the glasses and blinking. Each irLED was associated with its respective channel (PS1 measures the infrared pulsed from LED1, PS2 measures from LED2, PS3 measures from LED3) and the settings for current were chosen at the lowest level of $5.6 \mathrm{~mA}$ for each.

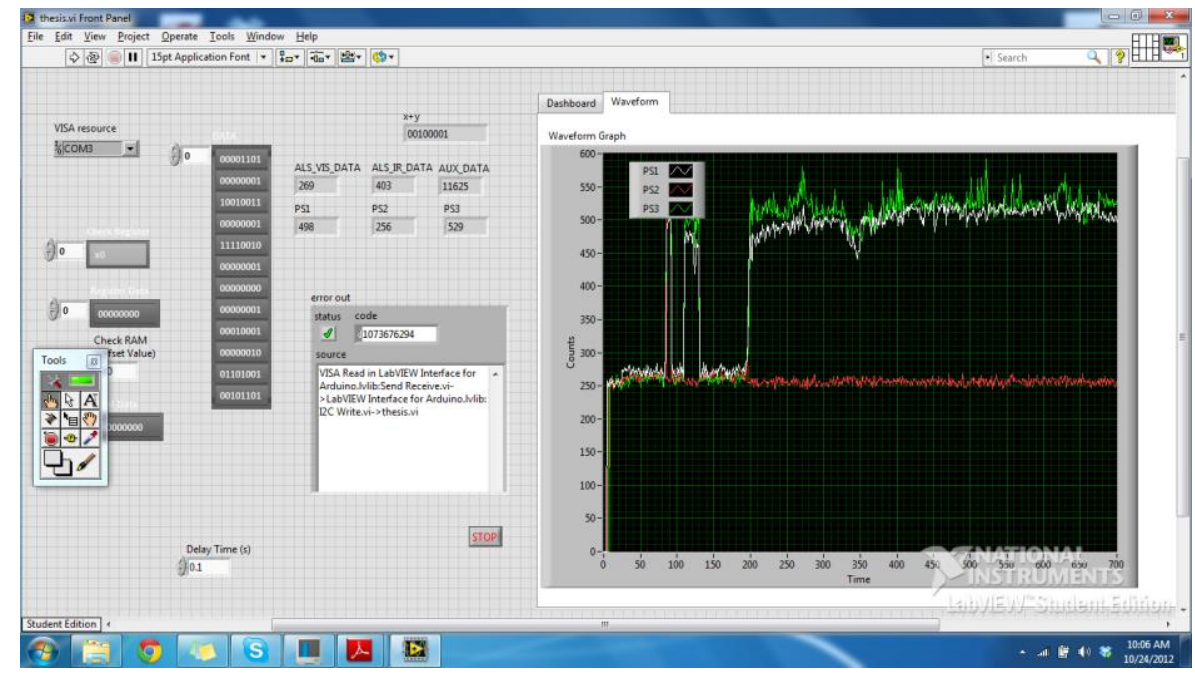

Figure 25. Eye Tracking LabVIEW Front Panel - The front panel shows the acquisition and plotting of the data in real time. The current drive for each LED was set at $5.6 \mathrm{~mA}$ during initial test runs. 


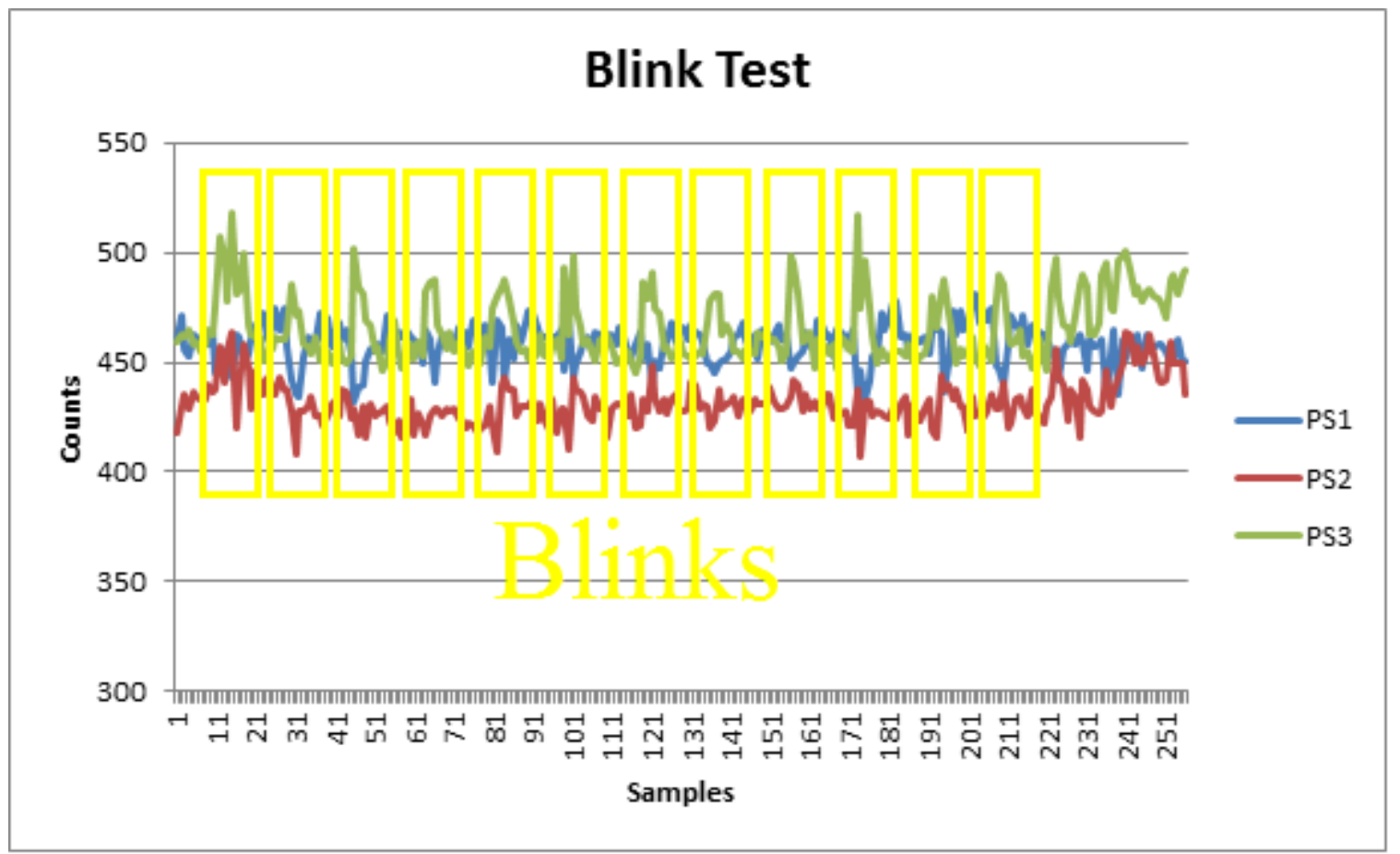

Figure 26. Blink Test Run - The graph shows three proximity sensing measurements taken when the user blinks. Each blink corresponded to a sudden spike in each of the PS channels.

The encircled areas of the test results show a strong successful correlation between the spikes of each PS channel to when the user was blinking. However, the \pm 10 count variance for each channel introduces a significant amount of high-frequency noise. Because the system was operating at such low currents and power levels, the resolution on the range of data was so low that inconsistent illumination from the irLEDs and ambient settings caused these fluctuations. In order to achieve a better signal to noise ratio, a higher current setting is recommended. By developing additional software, LabVIEW could process these signals to characterize them and detect them anytime a user blinks as shown in Section 4.7. 


\subsection{Printed Circuit Board Design}

After the first prototype was tested successfully, a more presentable and compact design of the system was sought after. This idea lead to designing the entire glasses out of PCB. Initial use of EagleSoft Light proved unfruitful due to the size limitation on their evaluation software. Therefore, ExpressPCB was used as an alternative to the design and fabrication process. Components and pads were designed to fit the parts selected for the glasses. Multiple pad placements around the frame for the irLEDs were designed to allow for a variety of test case configurations.

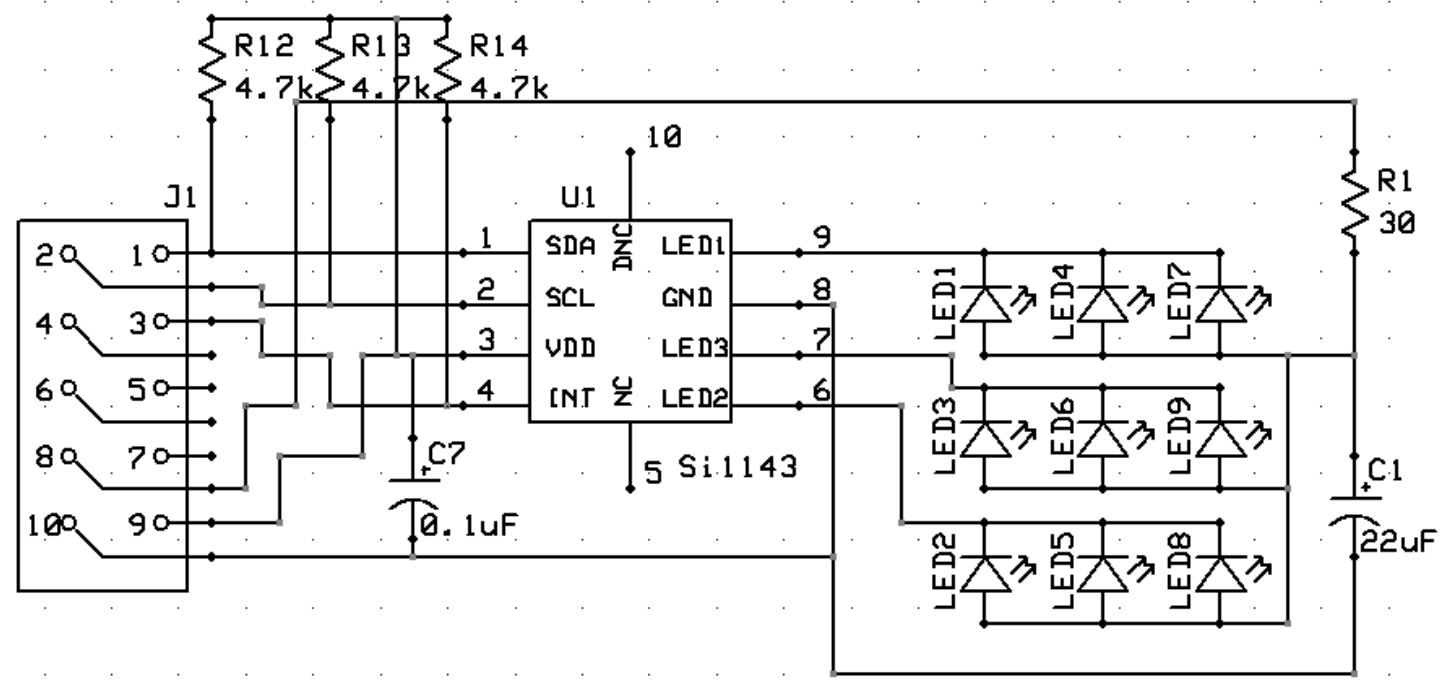

Figure 27. ExpressSCH Schematic of Si1143 Circuit - This schematic was linked to the ExpressPCB layout for easier routing. Multiple LED components were added to simulate multiple pad placements around the rim of the glasses.

Since most of the circuit operates at low current, trace width sizes were roughly estimated to 10 mils for a maximum of $300 \mathrm{~mA}$ operation. For traces with the connected irLEDs, the LED drive circuit of the Si1143 can pulse the current up to $359 \mathrm{~mA}$. Therefore, trace widths were conservatively increased to 15 mils for a maximum of 400 $\mathrm{mA}$ operation. Because this max value refers to average current, it adds an extra layer of 
security to the design of the board. Two test setups for eye tracking were designed on the left and right side portion of the glasses. Both circuits are almost identical with the exception of a routing mistake that caused LED2 and LED3 to be switched on the right side. This mistake has no huge effect as it only changes the order of the PS channels. Two rows of vias were also placed along the edge of the glasses to allow for easier cutting when the glasses was assembled.

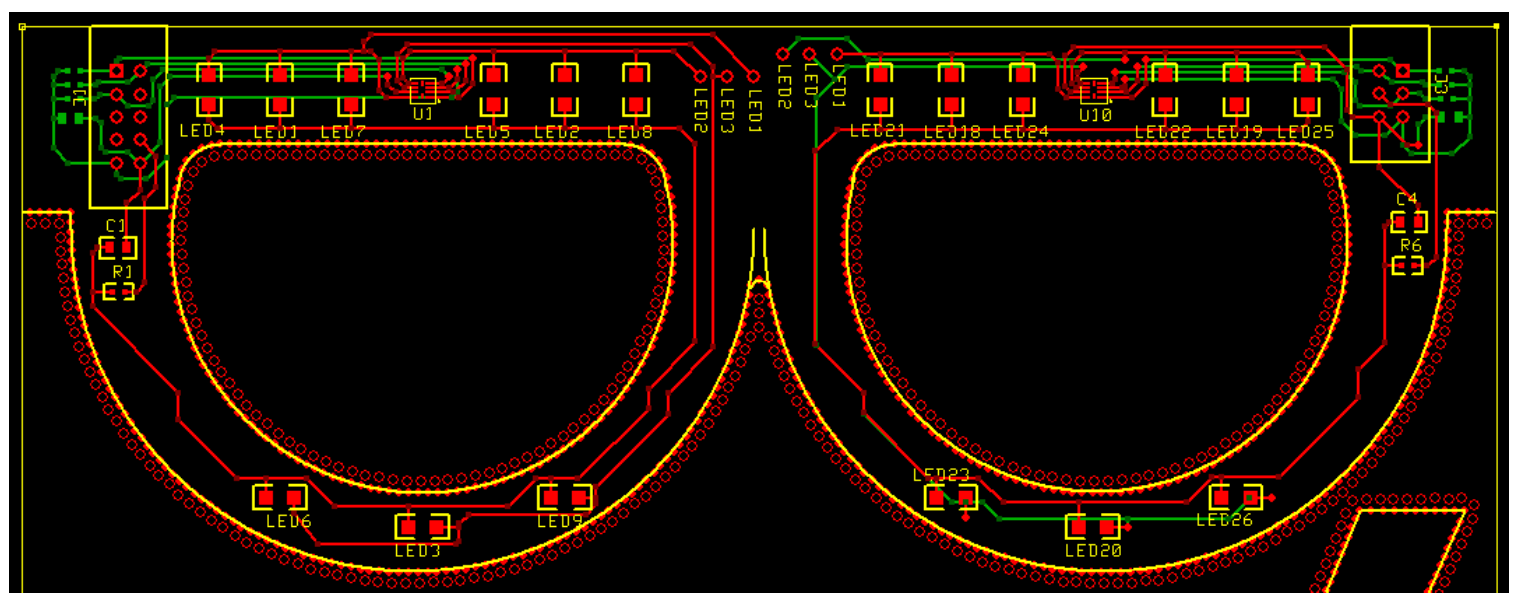

Figure 28. ExpressPCB Layout of Glasses Rim - The layout of the rims measured 16.3 $\mathrm{cm}$ in length and $6 \mathrm{~cm}$ in height. Multiple pads for the infrared LEDs were placed around the rims for multiple test configurations.

\subsection{PCB Assembly Procedure}

After the board was fabricated, the first step in the assembling process was to cut out the glasses rim pieces. The process is almost identical to the procedure outlined in Section 4.2 except that a 1/16" cut-off wheel in Figure 30 was used in place of the sanding drum. The vice grip held the PCB in place and the PCB was cut along the designated via holes. 


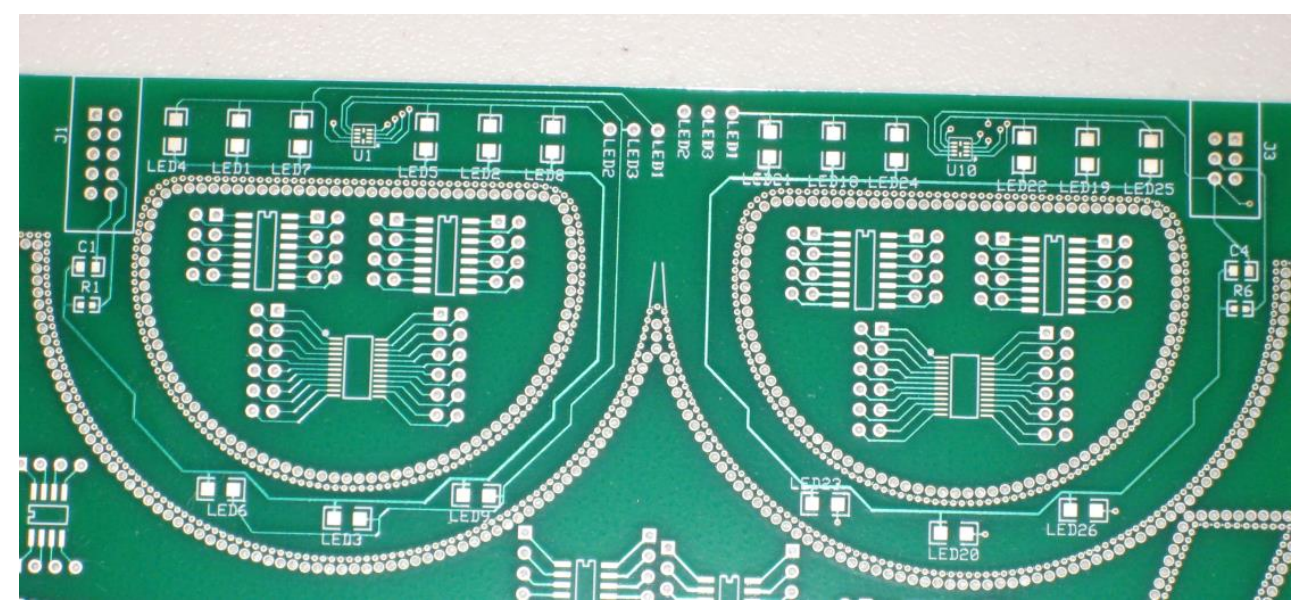

Figure 29. Fabricated PCB of Glasses Rim - This image shows the top layer of the fabricated PCB board.

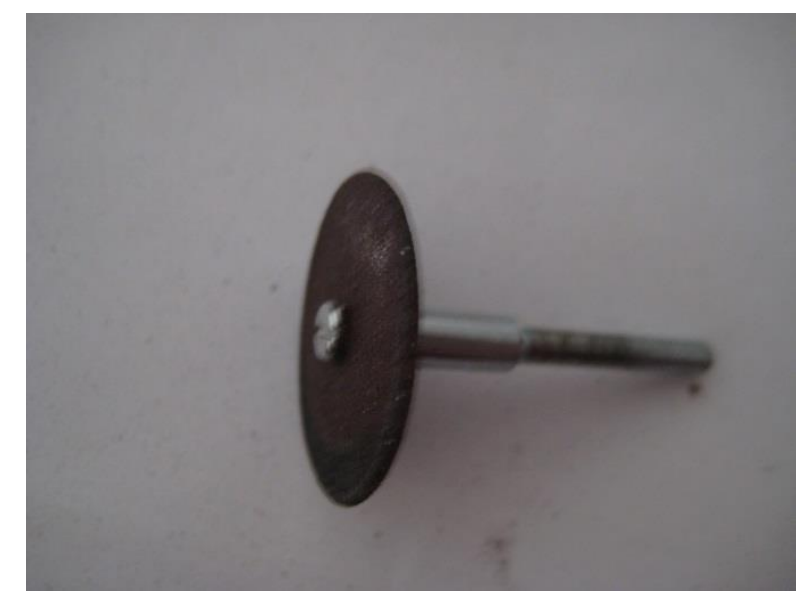

Figure 30. 1/16" Cut-off Wheel - This attachment was used instead of the sanding drum to cut out the fabricated PCB board.

Once all the pieces were cut out, a small jewelry box hinge in Figure 31 was used to connect the three pieces together. To adhere the hinge to the PCB material, a strong epoxy in Figure 32 was applied and set within 5 minutes. 


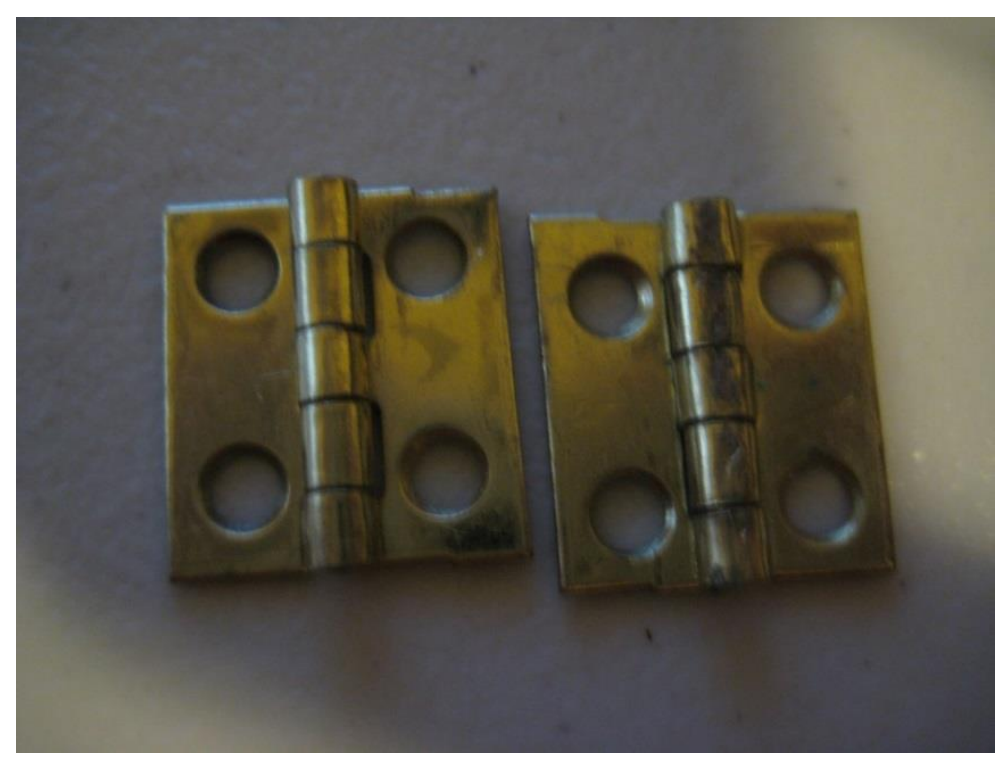

Figure 31. Schlage Jewelry Box Hinge - The hinge provided pivotal motion of the temple pieces.

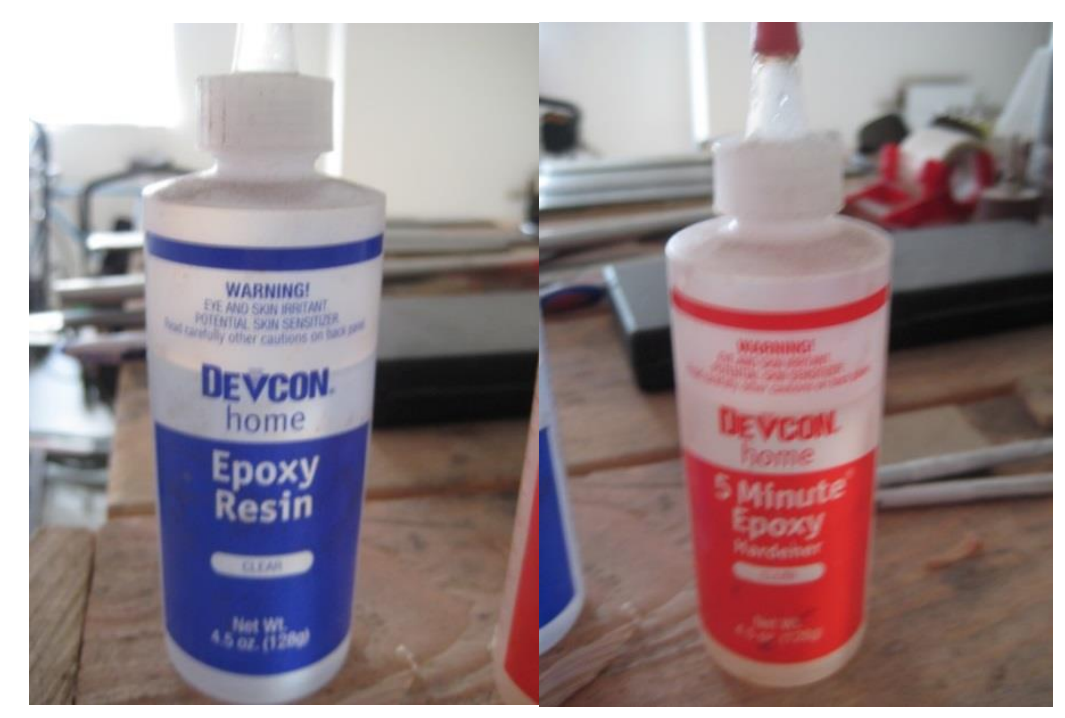

Figure 32. Devcon Home 5-minute Epoxy - This adhesive attached the hinge onto the PCB material. It was used as substitute for drilling screws into the PCB.

A SMD rework station was used to solder all the components onto the top layer of the board. Lead-free ChipQuik Solder Paste was applied to the surface of the pads and all the components were placed on top. By visually inspecting the pads and using a thermocouple to read the temperature, the hot air gun method was able to follow a typical 
reflow solder profile such as in Figure 33 for an effective solder job. This was accomplished by placing the hot air gun about 5 inches away for the initial pre-heat stage and bringing the hot air gun closer to about 3 inches to simulate the ramp up to the liquidus solder point. Once the solder melted and the components were set by the tension created from the liquid solder, the hot air gun was slowly moved away to simulate the ramp down stage. For this assembly process, a heat configuration setting of 6 was set on an Aoyue 906 Rework station.

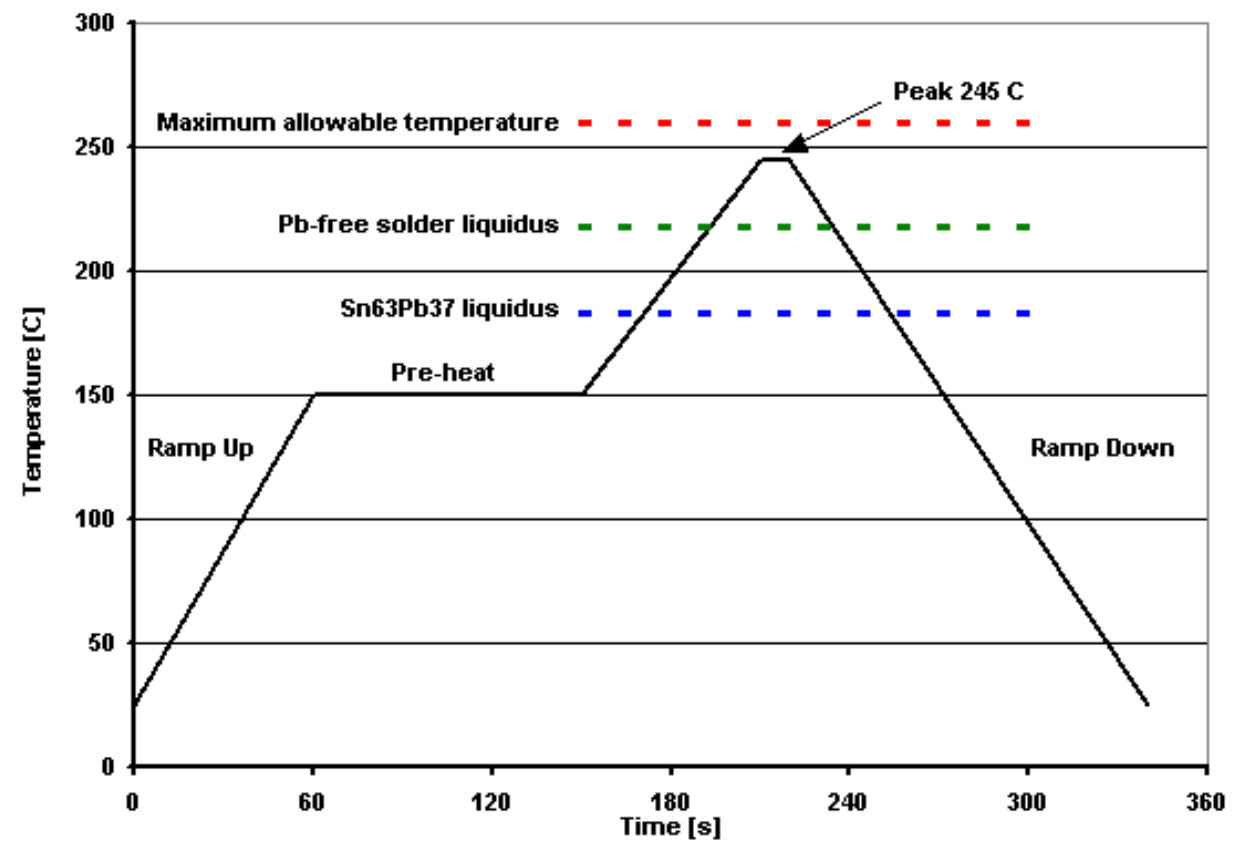

Figure 33. Typical Solder Profile - The temperature profile was roughly followed to ensure proper assembly of the components. This was done manually through the use of a hot air gun.

Some Instamorph plastic was used to mold the nosepiece and earpieces so that the glasses were comfortable to wear, stable on the face, and set to the right position. The final assembly is shown in Figure 34. 


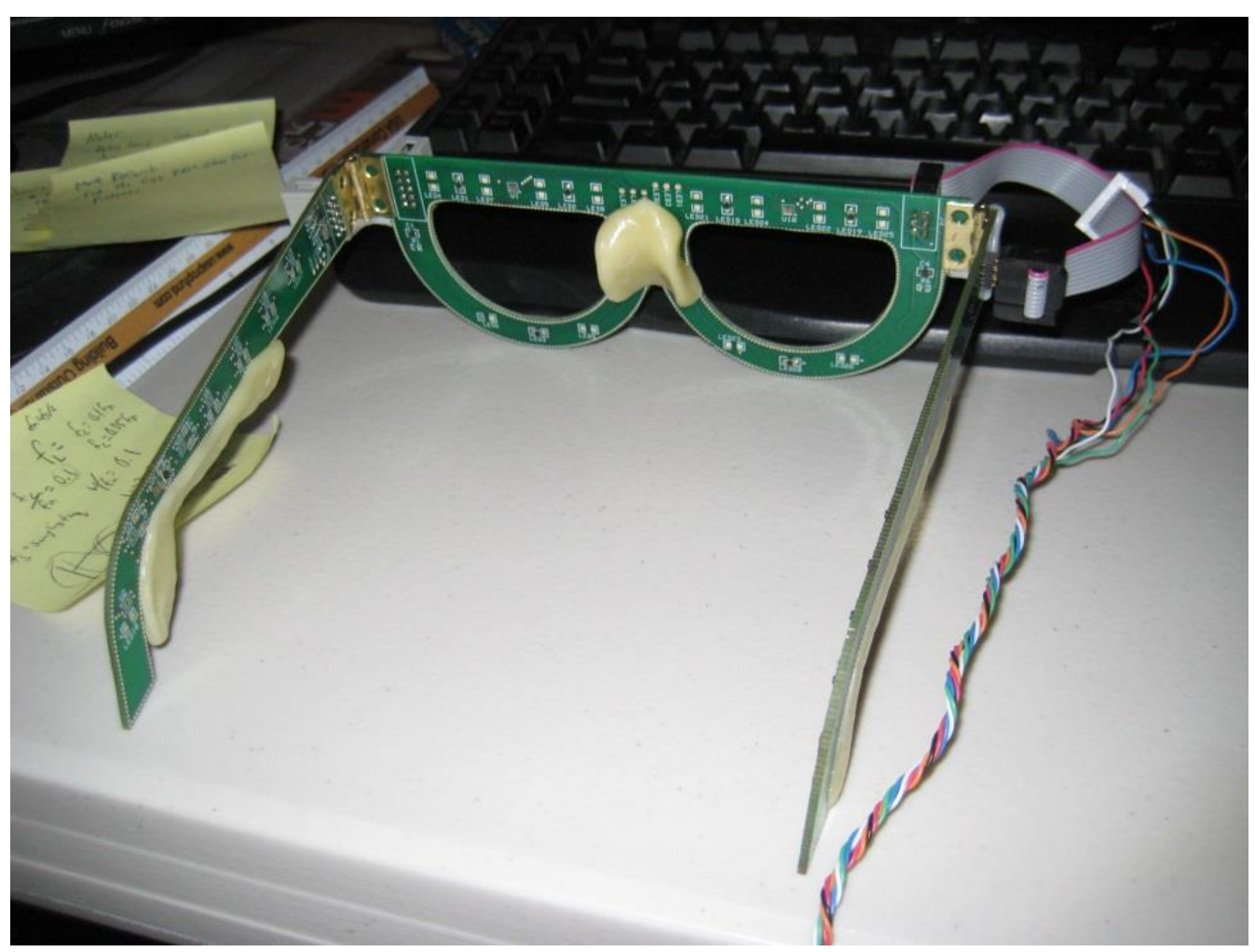

Figure 34. Assembled PCB Glasses Prototype - The final prototype was used to reconfirm initial findings and test the classification algorithm.

\subsection{Software Updates}

Much of the previous programming framework was kept intact when developing the LabVIEW VI for the PCB prototype. Section 5.5 mentions additional programming required to incorporate the heart rate sensor circuits. The only updates included an algorithmic solution to classifying the different eye movements. While the system is making measurements of the eye, the LabVIEW program is processing the data in realtime. Observations from previous test runs postulate that certain eye movements such as blinking, left glances, or right glances exhibit distinct characteristics in the Proximity Sensing measurements. 


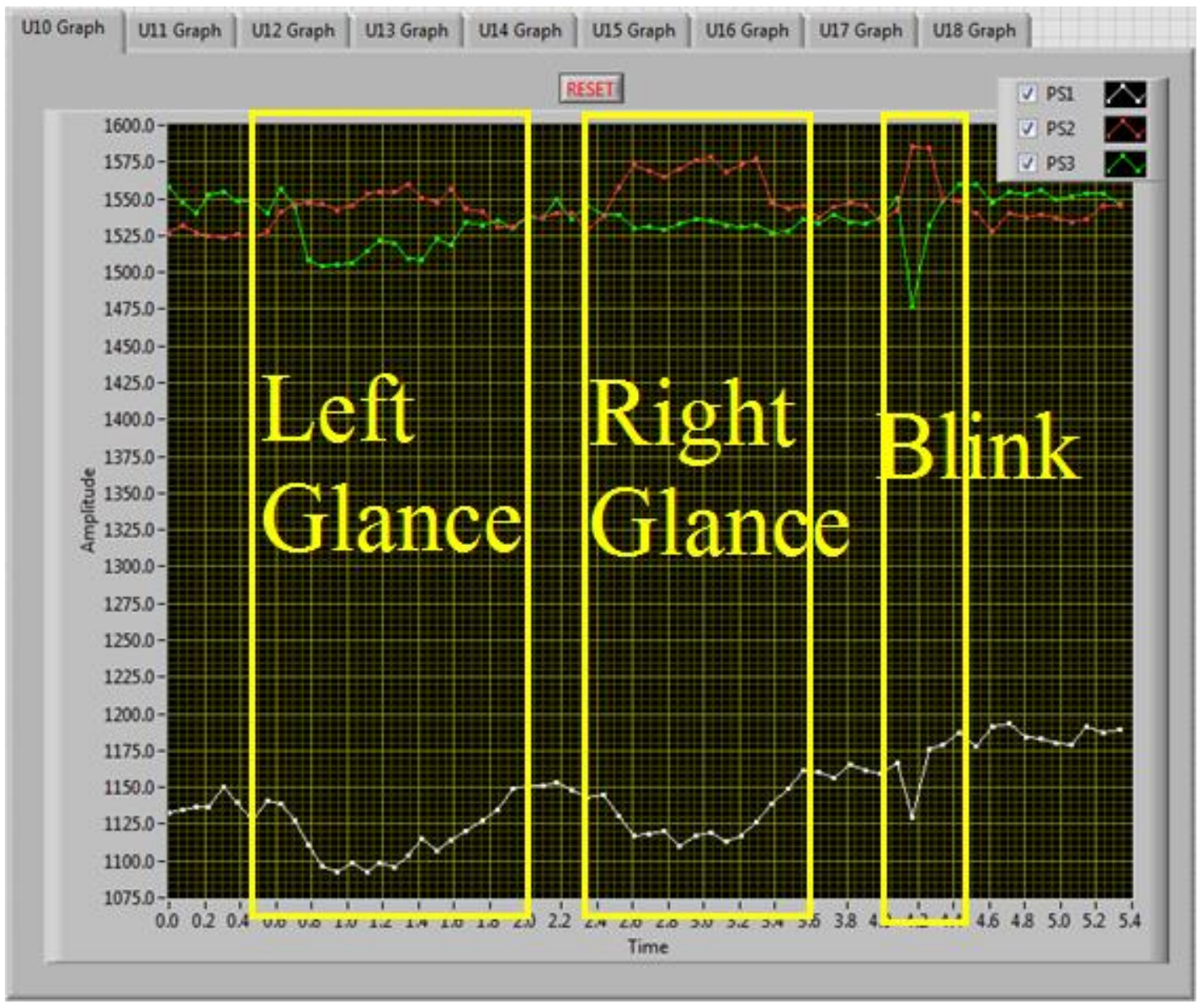

Figure 35. Left Glance, Right Glance, and Blink Test - Each movement shows a unique set of characteristics in each of the proximity channels. By identifying each of these, the correct movement can be classified.

Figure 35 captures a screenshot of the three motions mentioned. The left glance shows a decrease count in the PS1 and PS3 channels and an increase count value in the PS2 channel. The hill shaped or valley shaped waveform occurs as the eye moves in one direction, stays there, and moves back to the neutral position. The right glance shows almost the same changes in all the PS channels except in PS3. Blinking shows a sharp increase in the PS2 channel and a sharp decrease in the PS1 and PS3 channels. The waveform is then idealized to create a basis for an algorithmic solution to classify each motion. The idealized waveform is shown below in Figure 36. 


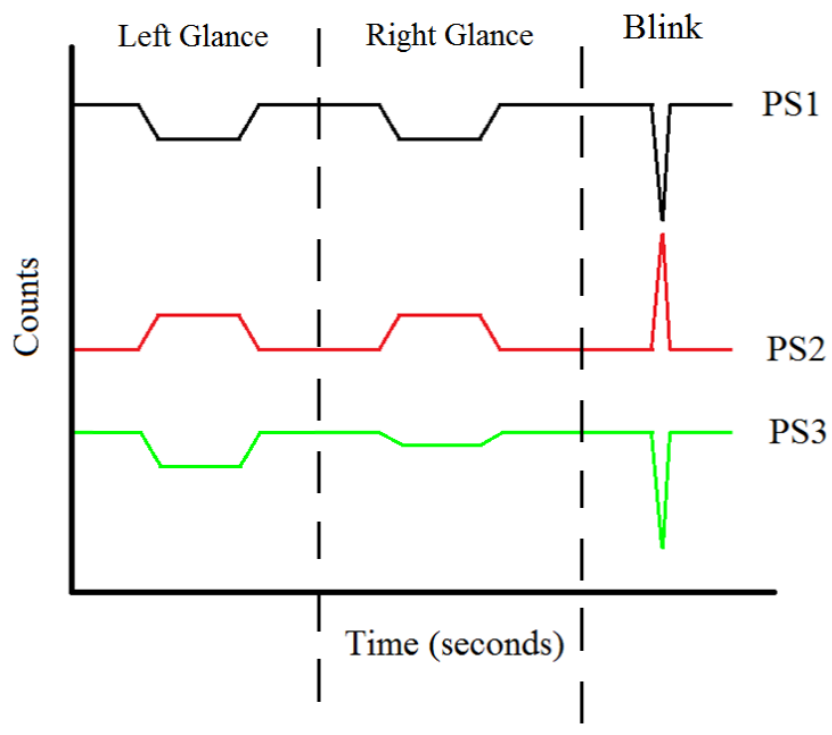

Figure 36. Idealized PS Waveform for Left Glance, Right Glance, and Blink Movement An idealized waveform was created based on previous observations. It served as a test basis for creating the classification algorithm.

The algorithm first inverts the PS1 and PS3 waveforms as in Figure 37 on the left. The three waveforms are then scaled and then added together to get the resultant waveform as shown on the right of the same figure. This new waveform is then processed two ways to achieve the desired detection response.

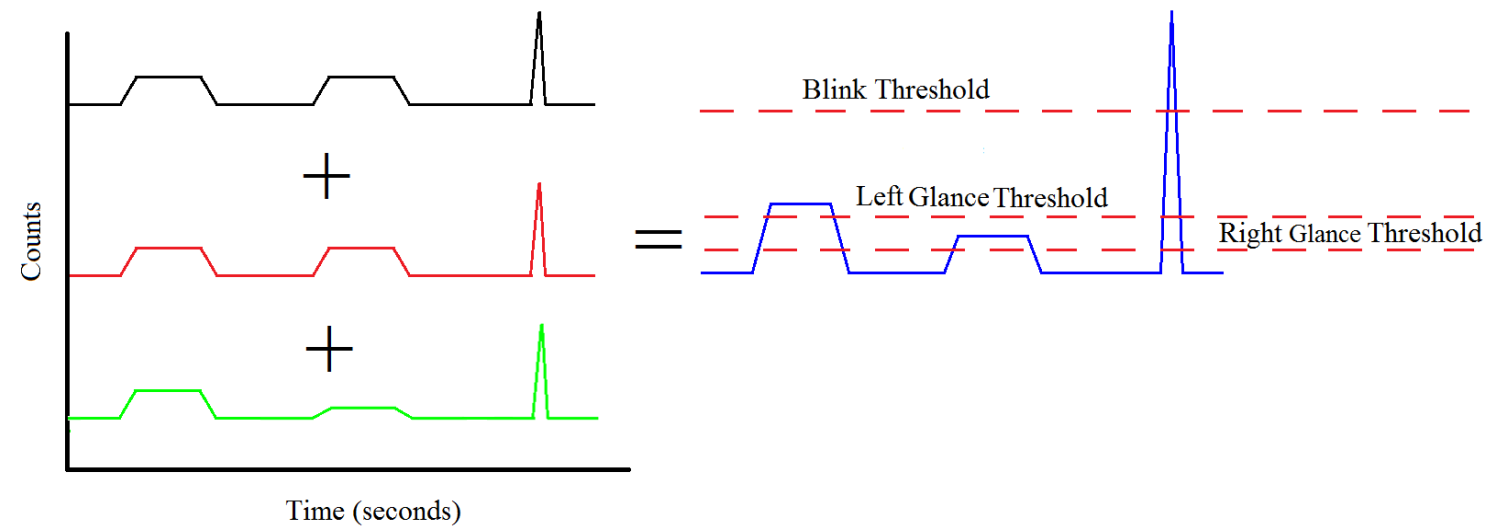

Figure 37. Graphical Algorithmic Solution - This figure visually displays the steps taken by the algorithm to identify each eye movement. 
For the blink response, the waveform is processed by passing the signal through a zero phase digital highpass IIR $7^{\text {th }}$ order Butterworth filter. The normalized cutoff frequency was set to 0.1 to filter out the baseline signal and lower frequencies. By using the butter() function in MATLAB, forward and reverse coefficients values for this filter in Table 5 were calculated and inputted manually into LabVIEW.

\begin{tabular}{cc}
\hline Reverse Coefficients $\left(\mathbf{a}_{\mathbf{k}}\right)$ & Forward Coefficients $\left(\mathbf{b}_{\mathbf{k}}\right)$ \\
\hline 1.000 & 0.492 \\
-5.589 & -3.443 \\
13.505 & 10.328 \\
-18.271 & -17.213 \\
14.937 & 17.213 \\
-7.374 & -10.328 \\
2.034 & 3.443 \\
-0.242 & -0.492 \\
\hline
\end{tabular}

Table 5. Reverse and Forward Coefficients for a 7th Order Butterworth Highpass IIR Filter (Normalized Cutoff Frequency $=0.1$ ). This filter removed the wandering baseline signal from the waveform to provide a better implementation of the peak detector.

The zero-phase filter is then implemented by running the signal through the IIR filter, reversing the output, running the output through another same IIR filter, and reversing the output again. By doing so, the output signal achieves zero group delay when comparing the original signal to the filtered one. This feature is important when trying to detect the blinking response in real-time. Since this type of filter can only operate properly on non-casual signals, the output time domain response is constantly changing as real-time data is coming in. However, the large values in the blink signals do not 
change much and usually stay above the set threshold value so the blink detection is fairly robust.

The left and right glance signals are processed by using the blink signal indices and removing the blink waveforms from the original signal. The signal is then smoothed through a moving average filter with a half-width of two. A peak detector VI is used to detect the left movement peak indices while another is used to detect both left and right movement peak indices. The right movement peak indices are then obtained by subtracting the left movement peak indices from the left and right movement peak indices.

\subsection{Final Experimental Results and Analysis}

All PS channels are enabled with their respective infrared LEDs. The current drive settings are as follows: LED1 at $11.2 \mathrm{~mA}$, LED2 at $67 \mathrm{~mA}$, and LED3 at $22.4 \mathrm{~mA}$. These values were chosen by trial and error to keep current consumption low while maintaining the same change in count range on each channel for each type of eye movement. Final experimental test runs of the glasses are shown below. Overall, the algorithm for the detection of blinking is fairly robust as long as the threshold is set high enough. Figure 38 shows a successful classification of the eye movements when the left and right movements are made once. 


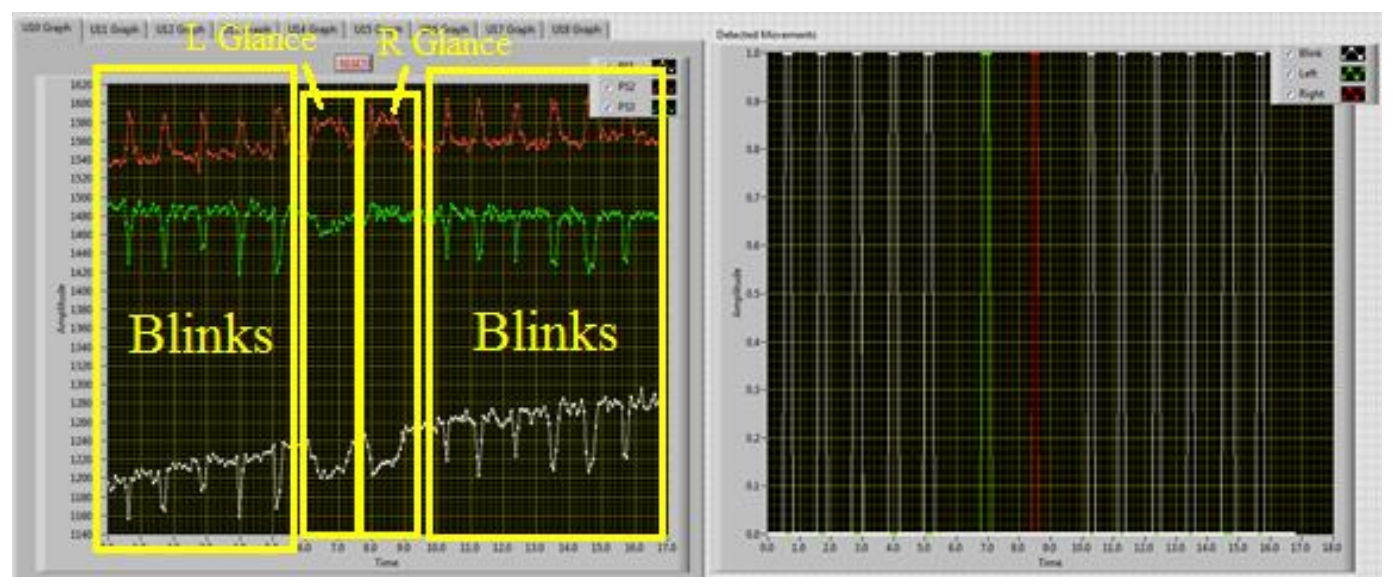

Figure 38. Successful Test Run of Tracking Eye Movement - This front panel VI shows a successful run of the measured PS channels on the left and the classified motion on the right. The test run started with five blinks, followed by a left glance and right glance, and ended with five more blinks.

However, the algorithm starts to fail when multiple left and right glances occur as shown by a test run in Figure 39. The sequence for this test run was blink, left glance, right glance, blink, blink, left glance, right glance, blink and blink. However, the detected movements show that a right glance happened at the same time as a blink and that an extra left glance occurred where a right glance should have.

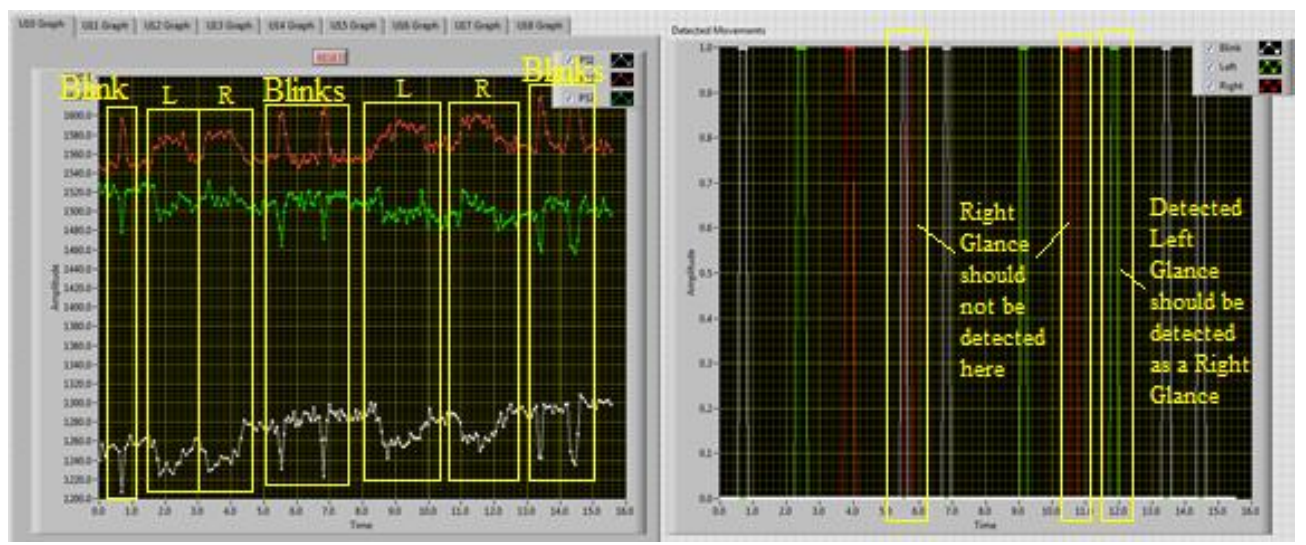

Figure 39. Unsuccessful Test Run of Eye Tracking Movement - This front panel VI shows an unsuccessful run of the measured PS channels on the left and the classified motion on the right. The eye movements for the test run are as follows: one blink, a left glance and right glance, followed by two blinks, a left glance and right glance, and ended with two blinks 


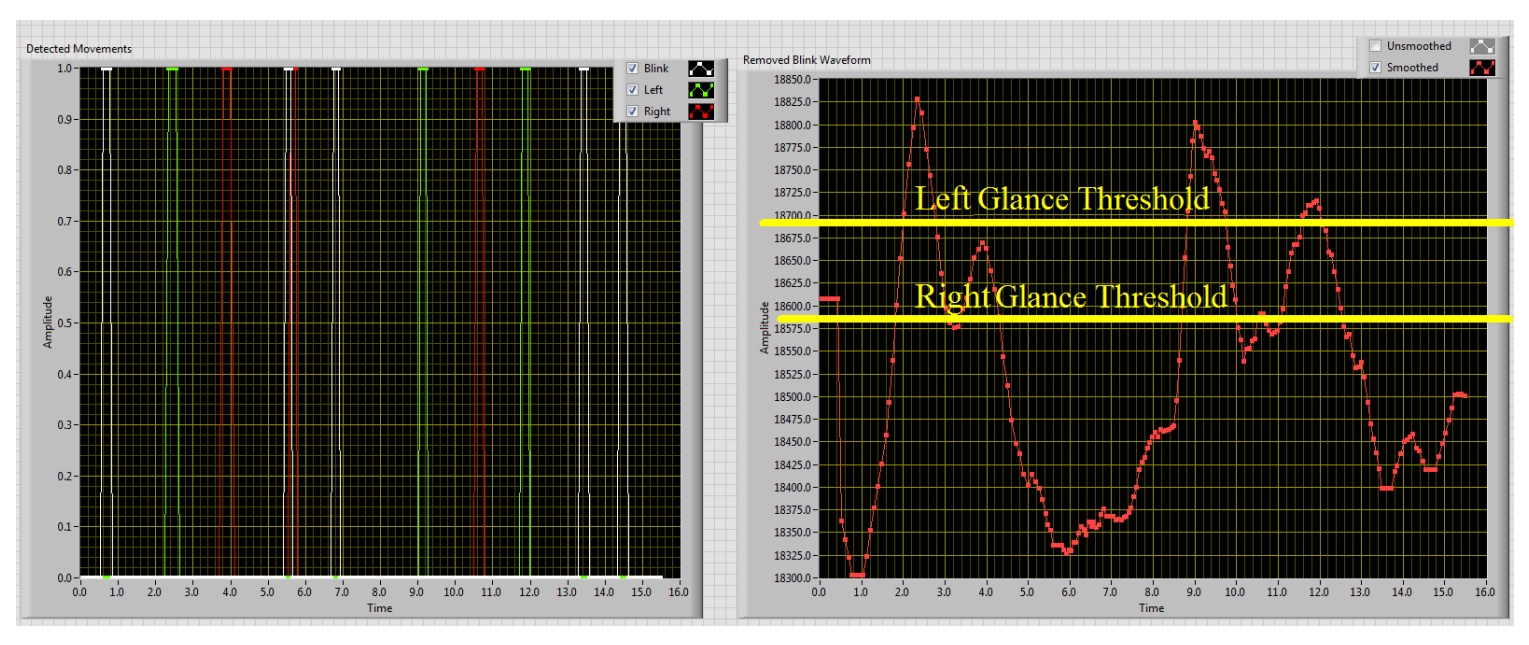

Figure 40. Analysis of Unsuccessful Test Run - Error resulted from the left and right glance detection algorithm. The right graph displays a visual explanation for the error.

Analysis of the "Removed Blink Waveform" in Figure 40 explains the result in the "Detected Movements" graph. Since the left glance threshold for detecting the peaks was too low, the peak detector VI mistakenly counted the fourth peak as a right glance. The right glance threshold was also low and detected the little blip in between the valley of the two peaks on the right side of the graph. These problems could be fixed easily if both thresholds were increased slightly. However, the values of those peaks are not necessarily the same every time the program is run so setting the thresholds becomes very arbitrary. The extra right glance on the blink also occurred because the algorithm did not remove the blink fast enough before the Peak Detector detected it. This observation raises some issues on the timing of the algorithm. 


\subsubsection{Experimental LED Power Consumption}

The oscilloscope images in Figure 41, Figure 42, and Figure 43 measure the voltage drop across the pulsed infrared LED, the time for that pulse, and the period before another pulse occurs. With these values along with Equation (17) from Section 3.2.2 and known current drive settings from the test run, the actual average power consumed by all three infrared LEDs can be calculated. Equation (21) displays the result of this calculation.

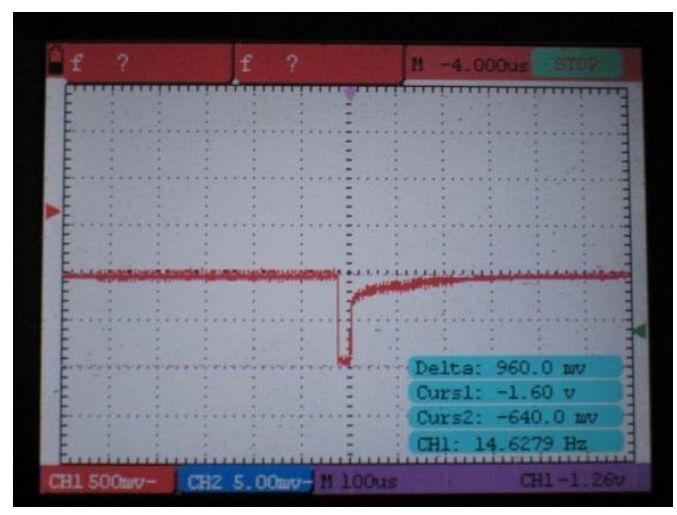

Figure 41. Voltage Drop Measurement across Pulsed LED. The cursors show a measured forward voltage drop of $1.6 \mathrm{~V}$ across LED1 of the PCB prototype glasses when driven. This value will be used to calculate experimental LED power consumption.

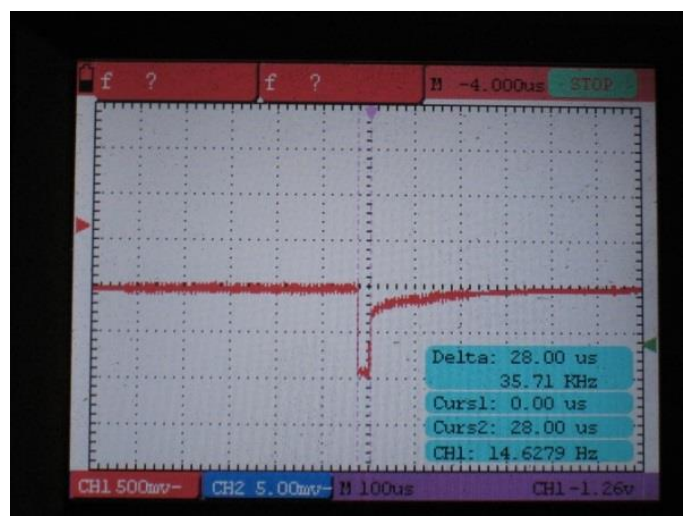

Figure 42. Time of Pulsed LED Measurement. The cursors measure the LED1 pulse width of the PCB prototype glasses occurring in 28 microseconds. This value will be used to calculate experimental LED power consumption. 


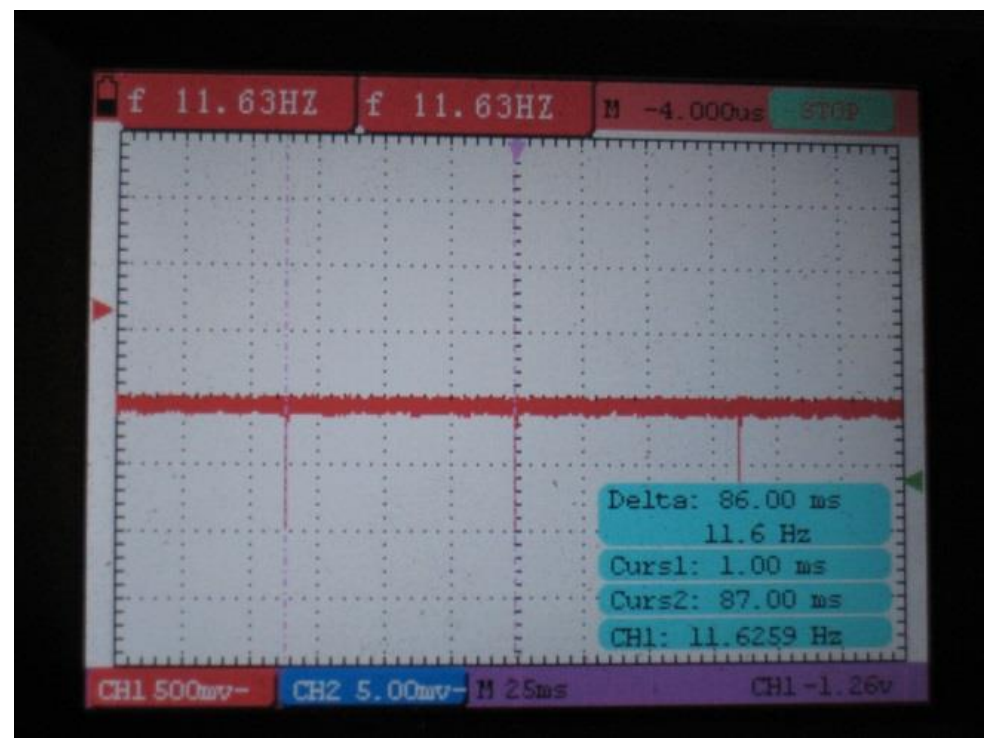

Figure 43. Time Period between Each Pulsed LED Measurement. The cursors measure the time between each LED pulse to be 86 microseconds. This value will be used to calculate experimental LED power consumption.

$$
\begin{gathered}
P_{\text {avg }}=\sum I_{\text {inst }} V_{\text {inst }} \cdot D=(11.2 m A+67 m A+22.4 m A) \cdot 1.6 \mathrm{~V} \cdot \frac{28 \mu \mathrm{s}}{86 m s} \\
=52.41 \mu \mathrm{W}
\end{gathered}
$$

The average power consumed by all three LEDs is substantially less than the theoretical average power of $13.5 \mathrm{~mW}$ as calculated in Section 3.2.2. This was a result of lower drive current settings, which was sufficient in detecting eye movement, and a longer time period between measurements, which happened as a result of the computer's execution speed of all the code. 


\section{CHAPTER 5. HEART RATE SENSOR}

This chapter explores using the Si1143 sensor as a heart rate sensor. The first half of this chapter focuses on the prototype tests and analysis that proves the concept of PPG. The second half attempts to implement the design onto a PCB glasses to integrate it into the whole development system.

\subsection{First Prototype Test Setup}

The heart rate sensor uses the same circuit schematic design as outlined in Section 4.1. Two setup configurations were used to test the feasibility of measuring correct heart rate data.

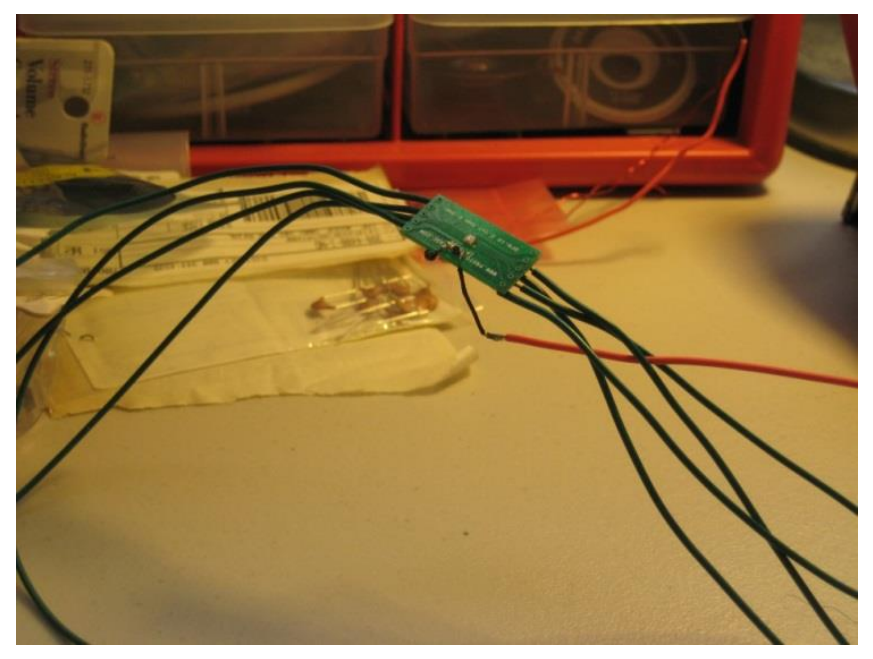

Figure 44. Reflection PPG Heart Rate Sensor Test Setup - The sensor is on the same plane as the LED and captures the reflectance off the blood vessels. Long wires were attached to this test setup to enable sensor placement on the Superior temporal artery.

The first setup used the transmission technique for PPG readings. This was done by placing the index finger between the irLED and the sensor as shown on the left of Figure 45 . The second setup used the reflective technique by placing the irLED $3 \mathrm{~mm}$ 
away on the same plane as shown in Figure 44 and on the right of Figure 45. The data and test results from each of these setups are shown in the next section.
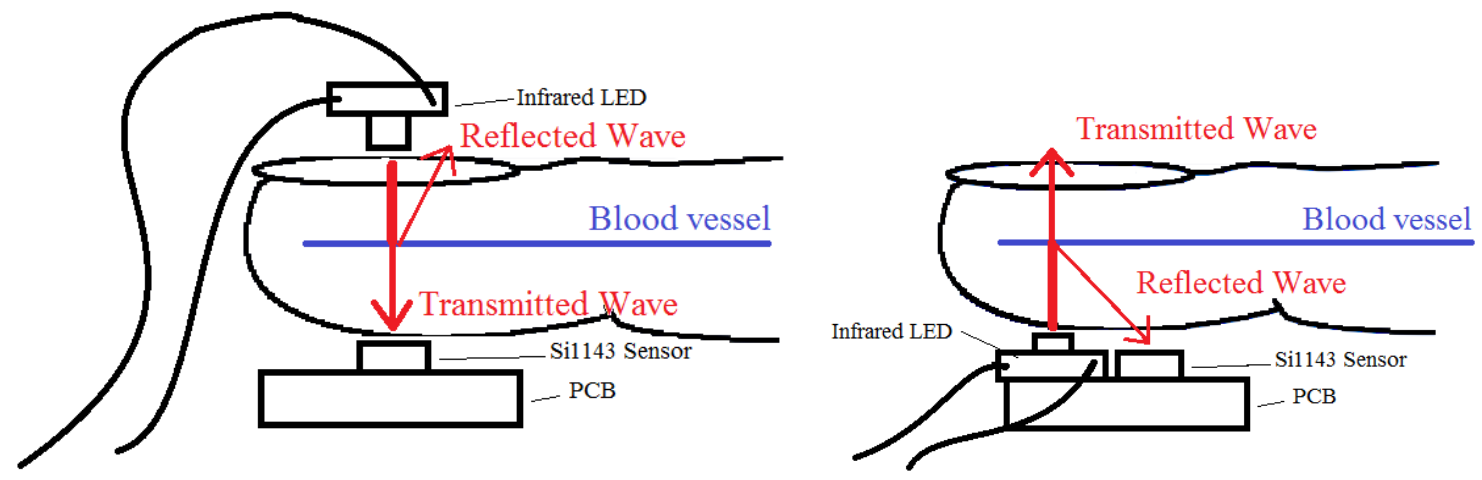

Figure 45. Transmission PPG (left) and Reflection PPG (right) - The transmission PPG captures the volumetric change of the blood vessels through the transmitted IR wave while the reflection PPG captures the volumetric change through the reflected IR wave.

\subsection{Initial Results and Analysis}

The next series of test results show use of the Si1143 sensor as a heart rate monitor through transmission and reflection method. Figure 46 above displays the front panel interface of the VI.

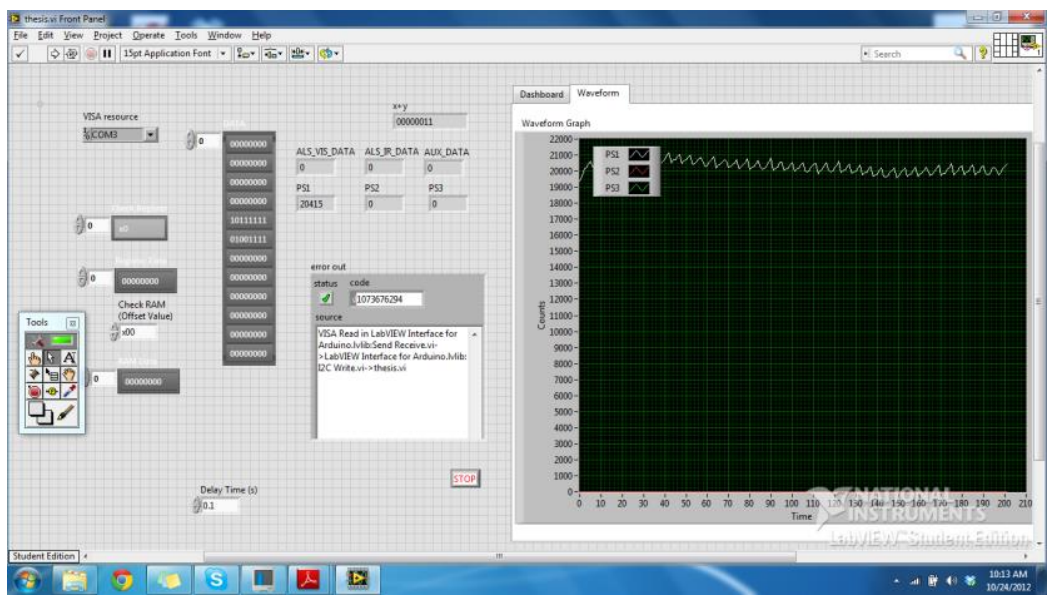

Figure 46. LabVIEW Front Panel VI for Heart Rate Sensor - This front panel interface shows the graph of an initial test run of the design. 
Figure 47 and Figure 48 are the results of a test case where the user measures their heart rate before and after mild exercise using a transmissive PPG setup on the index finger. From observation, the periodicity in the waveform strongly correlates to the beat of a human heart. The waveform also exhibits a slow-moving baseline which could be the result of finger movement during the measurement. Section 5.3 delves into a method for removing this wandering baseline and detecting the peaks programmatically.

Before the exercise, the heart rate measured 27 beats in 20 seconds or 81 beats per minute. Average adults at rest usually exhibit a heart rate around $60-80 \mathrm{bpm}$. Therefore, the initial results were within the correct range. The results after the warm-up show the heart rate measuring 43 beats in 20 seconds or $129 \mathrm{bpm}$. This increase in bpm shows that the sensor is detecting the heart rate signal.

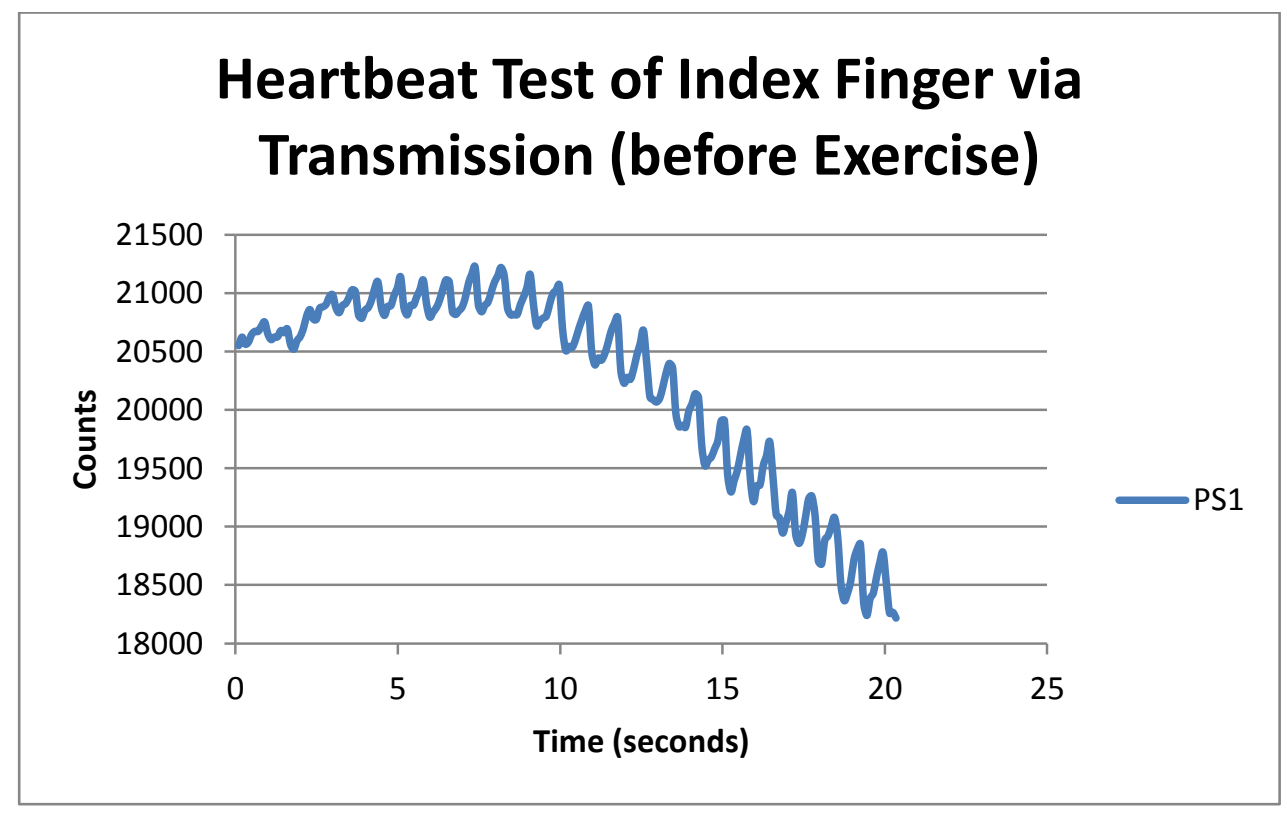

Figure 47. Heart Rate Test on Index Finger via Transmission Before Exercise - The graph shows a measurement of the PS1 channel on the index finger. The heart rate was measured through the transmission PPG method before mild exercise was performed. The results show an $81 \mathrm{bpm}$ rate. 


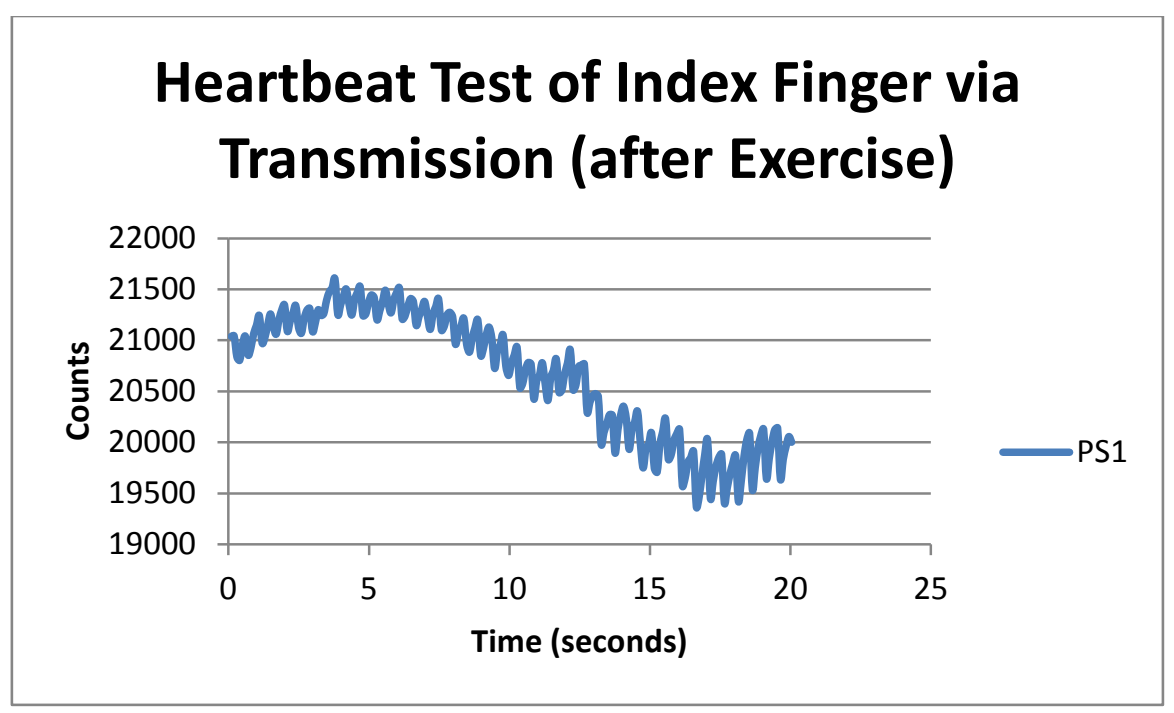

Figure 48. Heart Rate Test on Index Finger via Transmission After Exercise - The graph shows the an increase in frequency on the PS1 channel after mild exercise was performed. The same transmission PPG method was used to show an increased heart rate of $129 \mathrm{bpm}$.

The next series of tests in Figure 49 and Figure 50 attempt to prove the feasibility of reflective PPG on first the index finger and then on the superficial temporal artery as illustrated in Section 2.3.

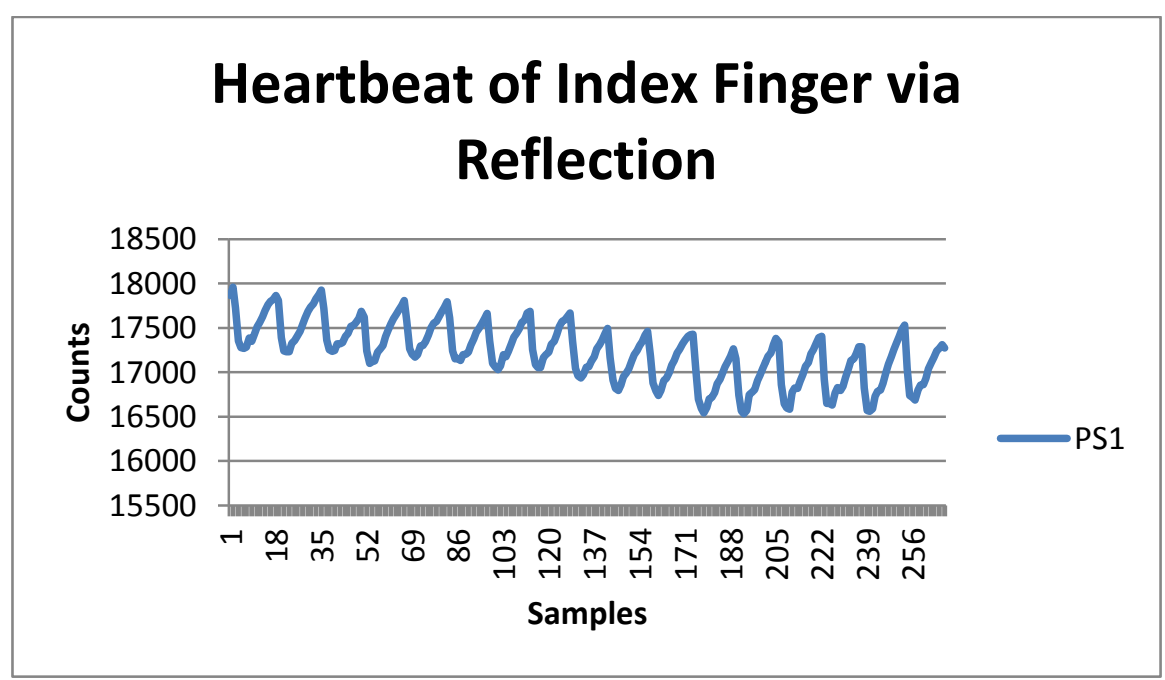

Figure 49. Heart Rate on Index Finger via Reflection PPG - The graph shows the results of a reflection PPG test run on the index finger. The waveform exhibits a consistent wave pattern as in the transmission PPG case. 


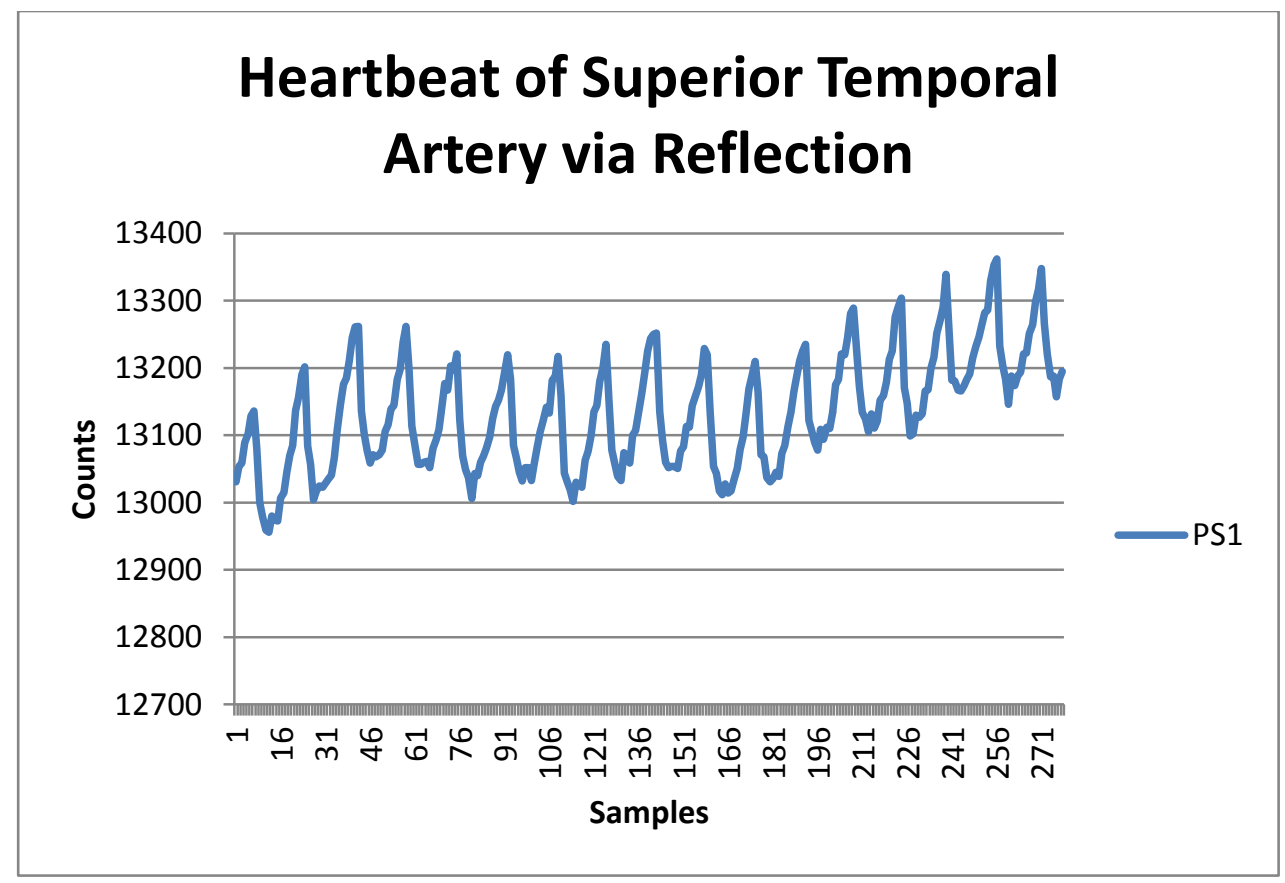

Figure 50. Heart Rate on Superior Temporal Artery via Reflection - The graph shows the results of a reflection PPG test run when the sensor is placed on the Superior temporal artery. The waveform shows that it is possible to get a reading of the heart rate.

Both the waveforms in Figure 49 and Figure 50 resemble the periodic waveforms in Figure 47 and Figure 48. Therefore, the results of the tests confirm that reflective PPG is also a possible method for detecting heart rate using the Si1143 Proximity/Ambient Light Sensor. Note that Figure 49 shows a big drop in baseline count magnitude from Figure 47. This is result of different positioning of the irLED to the sensor in the reflective and transmissive cases.

\subsection{Second Iteration of Software Design for Heartbeat Sensor}

The second design for the heartbeat sensor included a portion of the code to process the signal and display a digitized heartbeat in real-time. If the signal had a constant baseline value, the detection and digitization of the heart beat signal would be simplified to a comparator circuit or threshold detector. Since this was not the case, a 
circuit (Figure 51 courtesy of Dr. Vladimir Prodanov) was implemented in order to combat the effect of a moving baseline. The hysteresis comparator compares the original signal with a smoothed, slightly-delayed version of itself. Whenever the original signal passes above the filtered signal (plus the hysteresis), it outputs a digital logic high which represents a heartbeat.

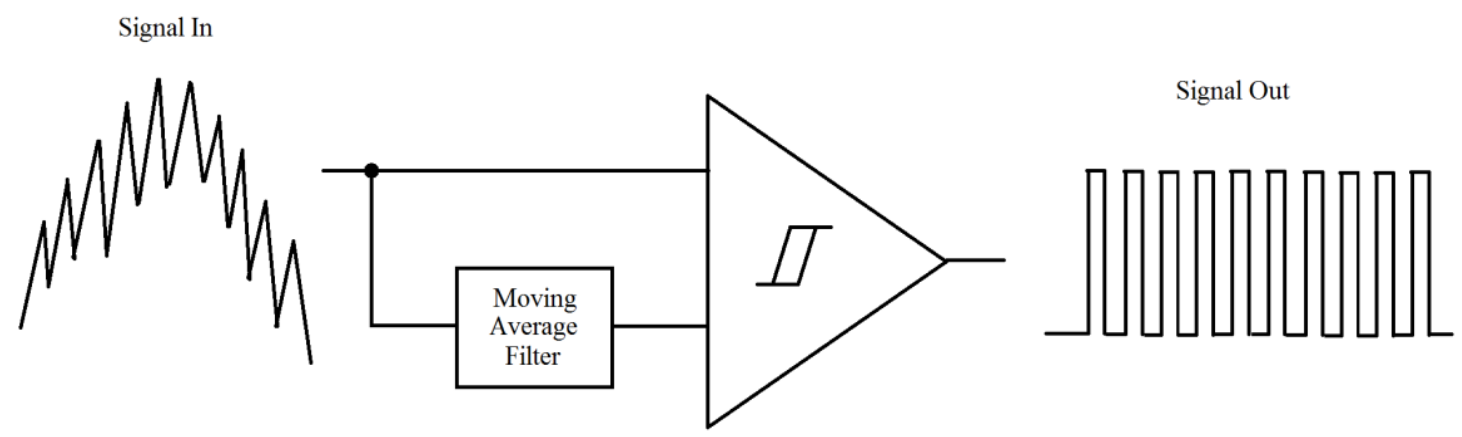

Figure 51. Moving Average Hysteresis Comparator - This circuit was implemented in the program to detect the peaks of the waveform and convert them to a digital signal.

The software version of the analog circuit shown above was implemented in LabVIEW. The signal is compared with a moving time average of itself in a hysteresis comparator. By applying a moving average to the signal, it smoothes out the signal and resembles the moving baseline waveform. Using a hysteresis comparator ensures that any spurious noise on the original signal does not affect the circuit from detecting the signal crossing the baseline. With this function created in LabVIEW, the program was successfully able to detect the heart rate signal. The results of a sucessful test run on the LabVIEW Front Panel is shown in Figure 52 where the indicator for the beat counts and BPM displays a value of 35 beats in about 32 seconds resulting in a $65.7 \mathrm{bpm}$ rate. 


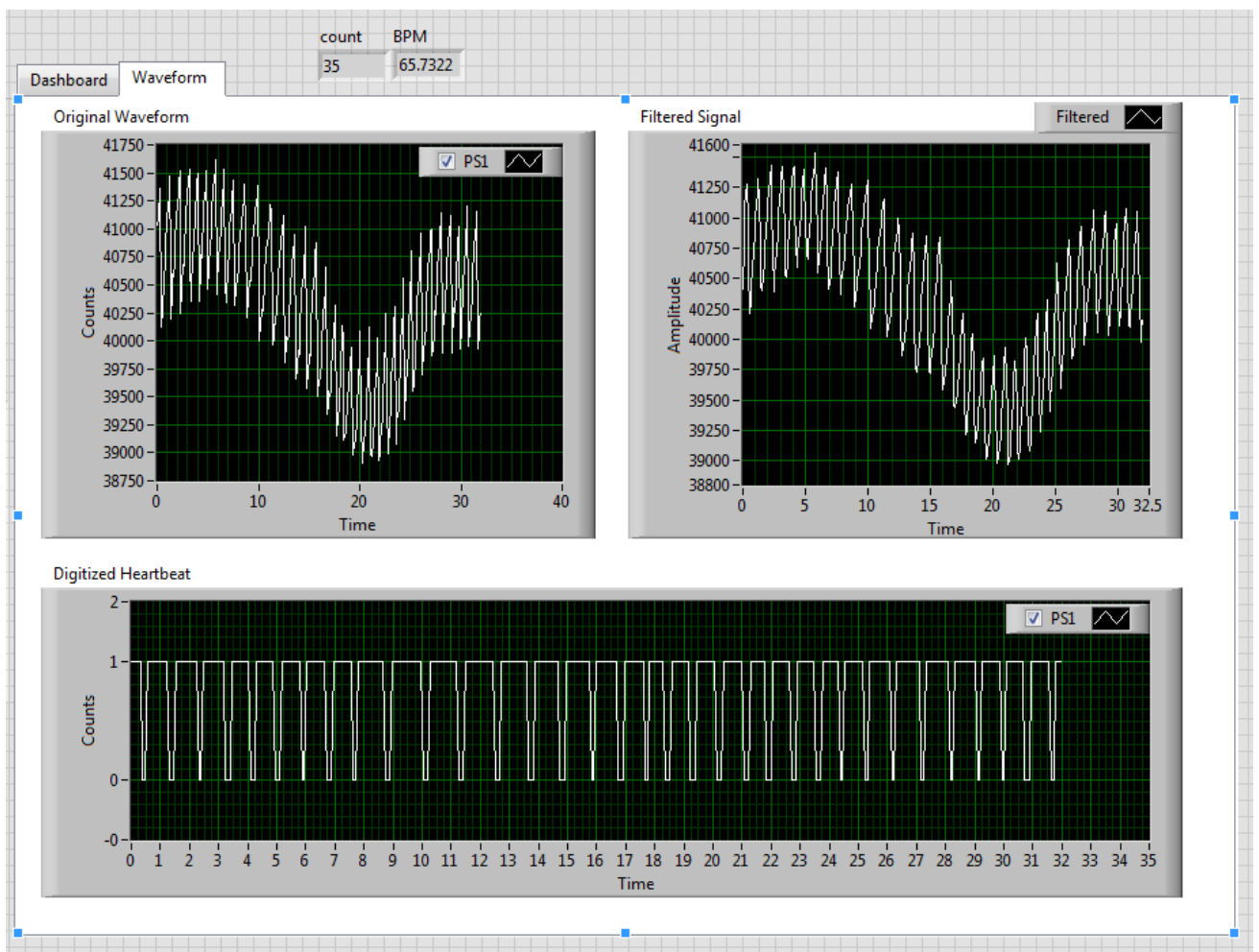

Figure 52. Front Panel of Heart Rate Test Run with Digitized Count - This front panel image shows the result of implementing the hysteresis comparator circuit in LabVIEW. Each corresponding digital high output represents when the heart beats.

\subsection{PCB Design}
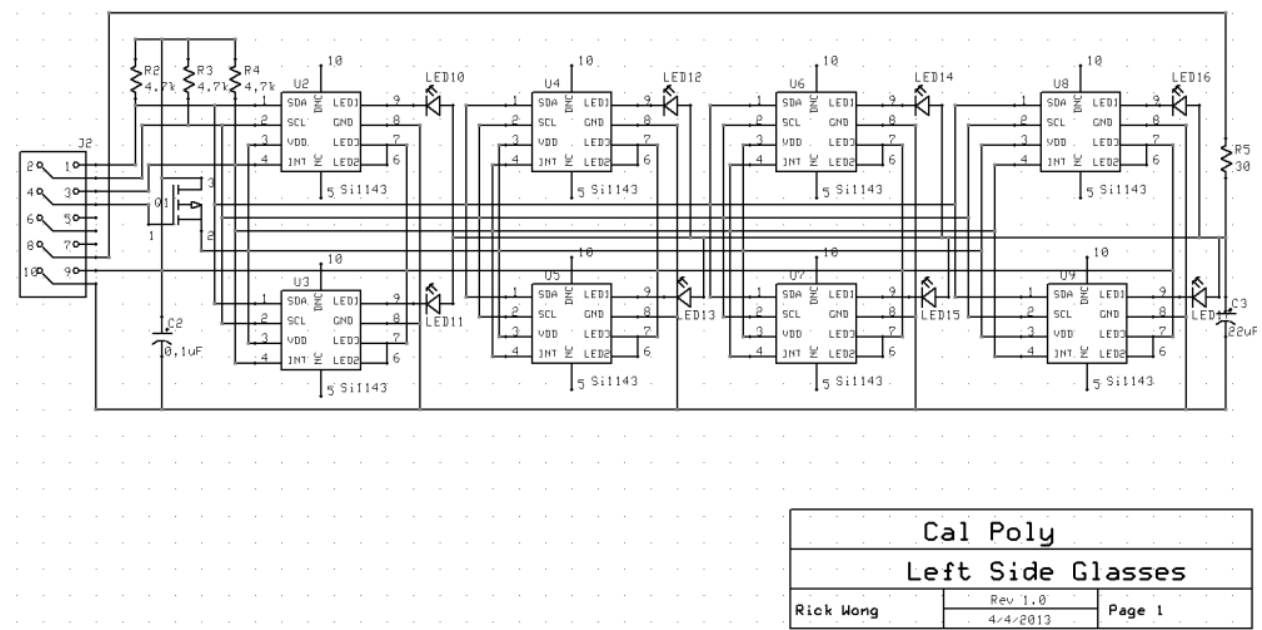

Figure 53. ExpressSCH Schematic for Left Temple Piece - This schematic is modified from the eye tracker circuit to enable multiple placements of the sensor around the left temple piece of the PCB glasses. 
After initial testing showed promising results in the superior temporal region, the next step involved incorporating the Si1143 sensor on the temple pieces of the glasses and measuring it only through reflection PPG. Pinpointing the exact location where the sensor can pick up signals from the superior temporal or posterior auricular arteries was too difficult to design for because of different head shape sizes. As a result, the left temple piece features multiple pad placements for the Si1143 sensor and a single irLED to increase the chance of detecting the signal from the user. Each site is connected in parallel to all the other sites so only a single sensor and irLED can be soldered on at one time.

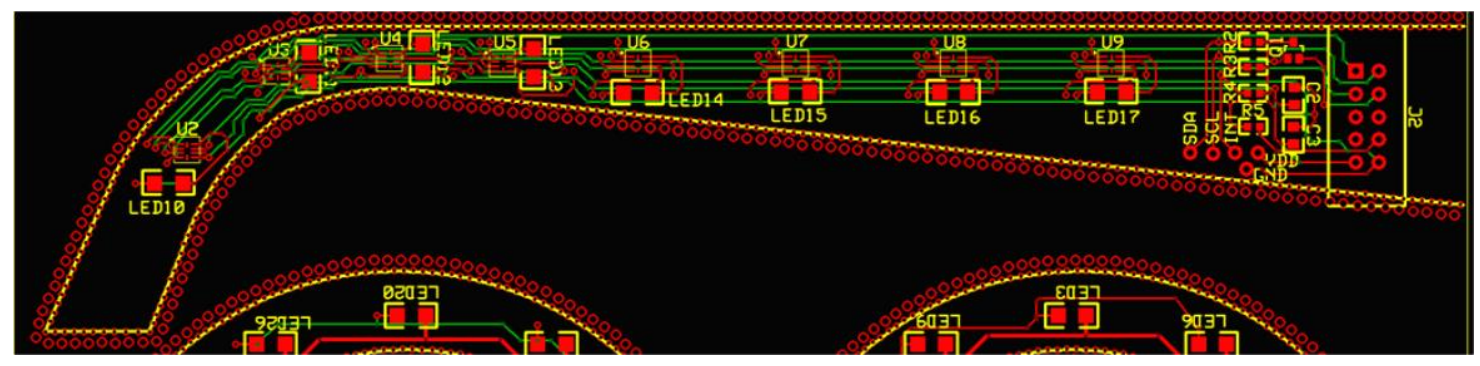

Figure 54. ExpressPCB Layout of Left Temple Piece - This layout shows the pad placements and routing for all the components specified in the left side heart rate schematic.

By using this design, both the eye tracking circuit and the heart rate sensor circuit are connected to the same $\mathrm{I}^{2} \mathrm{C}$ bus that enables one software program to communicate with both sensors. However, because all Si1143 sensors contain the same slave address upon startup, modifications of the $\mathrm{I}^{2} \mathrm{C}$ address through the RAM parameter settings need to be made. In order to interface the two Si1143 sensors on the same $\mathrm{I}^{2} \mathrm{C}$ bus line, one sensor must be turned off while the other has their $\mathrm{I}^{2} \mathrm{C}$ address modified. This operation was done through the use of a generic P-channel 20V 3.1A MOSFET as a switch for the VDD line of the Si1143 acting as a heart rate sensor. It should be noted that there was an 
error in pad placement for the gate, drain, and source of the switch MOSFET. This was easily remedied by orientating the transistor correctly and using a nearby via as a substitue pad.

One design flaw of the left temple piece is that constant desoldering, soldering, and testing would be involved if the sensor could not detect the heart rate on that pad. Since there are two sides to a pair of glasses, the right temple piece was designed for running multiple sensors at once as an auxiliary test setup to the left piece.

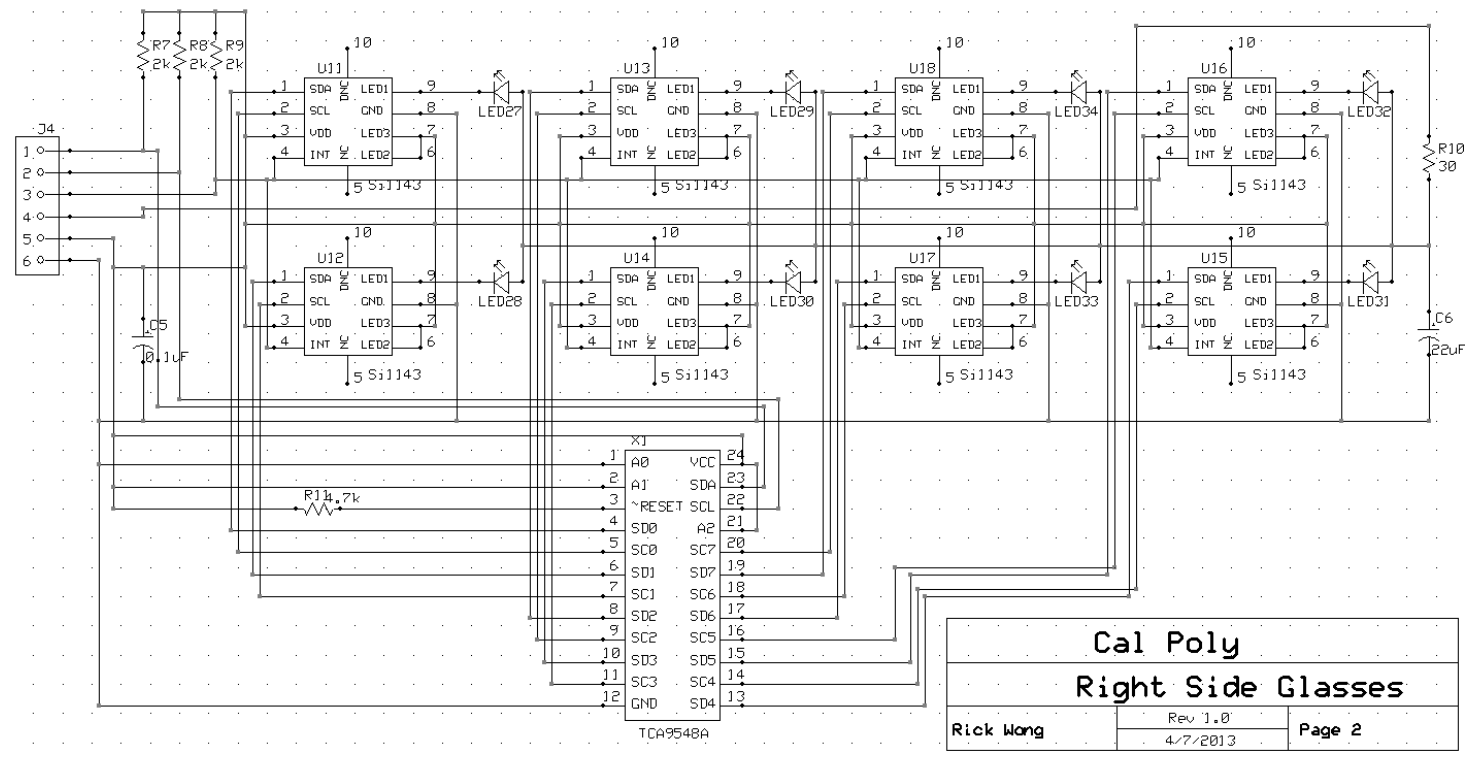

Figure 55. ExpressSCH Schematic of Right Temple Piece - This schematic is modified from the eye tracker circuit to enable the use of eight sensors to measure heart rate.

\section{By utilizing Texas Instruments TCA9548A Low Voltage 8-Channel $\mathrm{I}^{2} \mathrm{C}$ switch,}

the design is able to interface with up to $9 \mathrm{Si} 1143$ sensors and activate or deactivate any one sensor. This design also eliminates the need for an extra pin from the Arduino to drive the FET switch since the $\mathrm{I}^{2} \mathrm{C}$ switch also operates on the same $\mathrm{I}^{2} \mathrm{C}$ bus. 


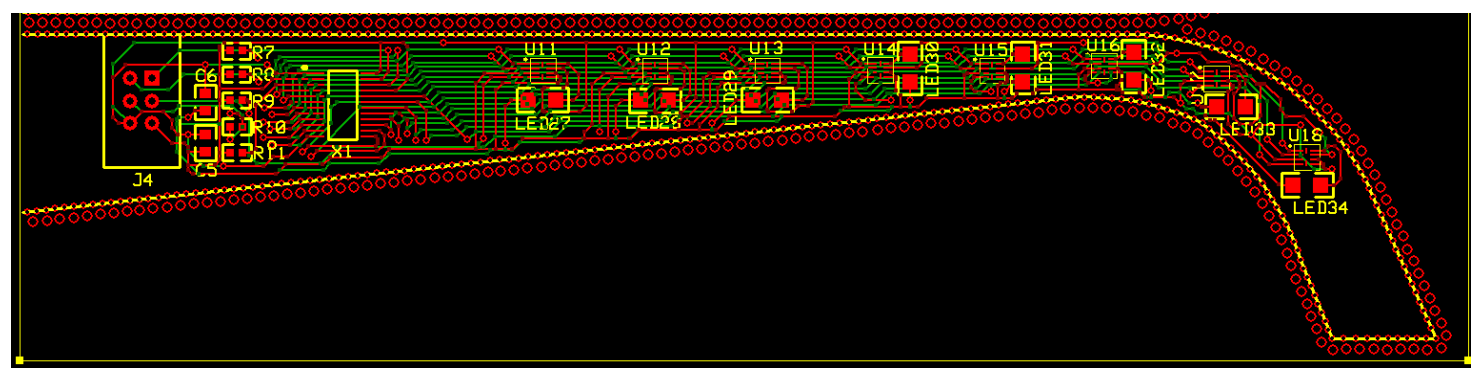

Figure 56. ExpressPCB Layout of Right Temple Piece - This layout shows the pad placements and routing for all the components specified in the right side heart rate schematic.

\subsection{Software Updates}

Modifications were made from the eye tracking program in Section 4.7 to accommodate operating up to 9 sensors at once. With the use of Texas Instruments TCA9548A Low Voltage 8-Channel I² C switch, each sensor's SDA and SCL were made to turn on one at time so that the sensor could be initialized and have their initial $\mathrm{I}^{2} \mathrm{C}$ slave address changed. This task is accomplished by writing a byte signal to the TCA9548A. Each bit in the byte represents the on or off state of each sensor's SDA and SCL lines. The slave address $0 \times 76$ of the $\mathrm{I}^{2} \mathrm{C}$ switch was predetermined by hardwiring the slave address pins of the chip in the design of the PCB board. 


\subsection{Final Results and Analysis}

Each sensor on the right temple piece was tested individually to determine if the heart rate signal could be detected. Unfortunately, all of the sensors were unable to make a successful measurement. Figure 57 shows a common result from one of the sensors.

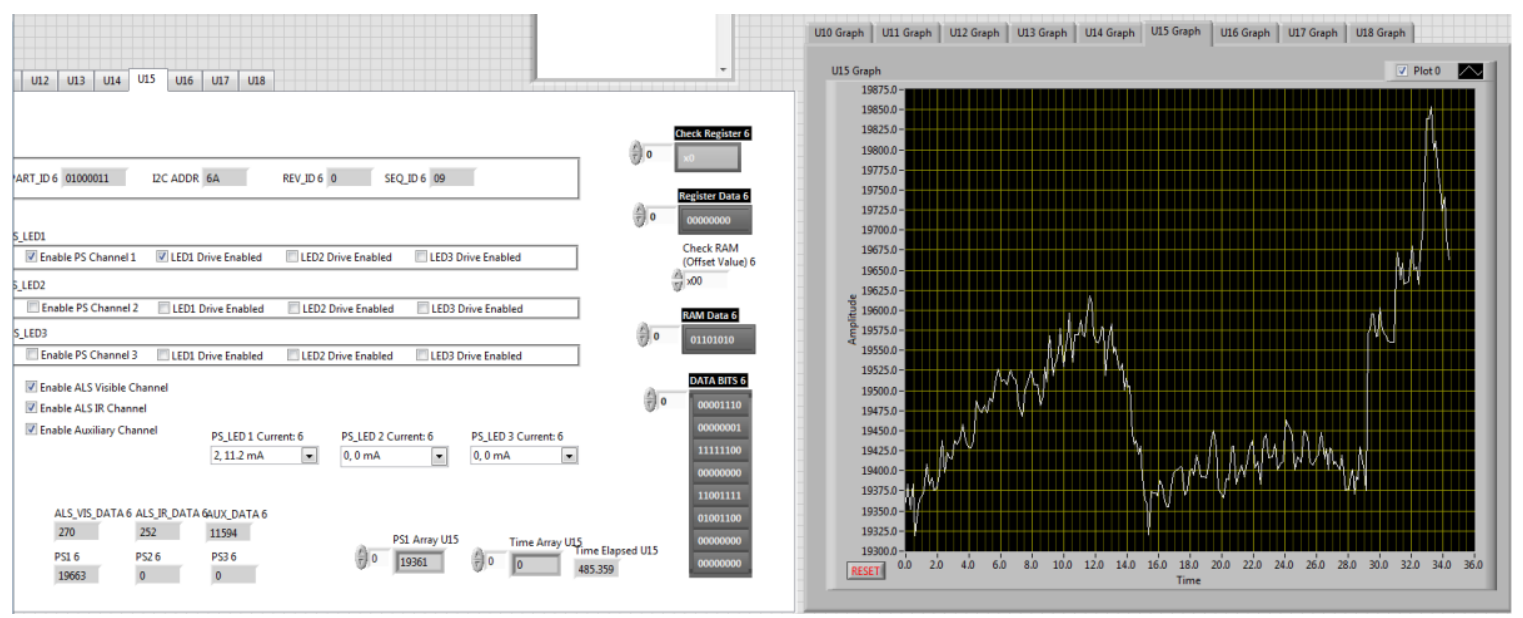

Figure 57. PCB Test Run of Heart Rate Sensor - This front panel image captures a typical test run for the final PCB prototype design. The graph on the right shows no distinguishable, consistent waveform as seen in previous tests.

The results show that the sensor detected a lot of random signals that was not deterministic of a heart rate signal. In analyzing the cause of these failed tests, several criteria were observed when compared to the successful test cases of Section 5.2.

The first criterion is the placement of the sensor with respect to the blood vessel. If the sensor does not lie directly above a blood vessel, the sensor will have a difficult time getting a measurement. The second criterion is the mechanical stability of the glasses. Movements of the head caused the temple pieces of the glasses to move abruptly and create sudden changes in the signal. In order to fix this issue, a better frame or a tighter fit of the glasses may increase its mechanical stability. The third criterion is the 
absence of obstruction between the sensor and the skin. The sensor needs direct contact with the skin to operate optimally. In these cases, the hair got in the way of achieving an accurate reading. The best solution to all these issues would be to attach the infrared sensor and the LED to the earlobe and get a measurement from there. However, this solution has already been investigated in other studies [15]. This method of placing the heart rate sensor on the temple pieces should not be abandoned since further mechanical improvements made to this design may improve chances of measuring an accurate reading. 


\section{CHAPTER 6. CONCLUSION}

\subsection{Summary of Investigation}

This thesis explored the Si1143 proximity/ambient light sensor as a possible method for detecting the eye as well as measuring the heart rate of the human body. The main outcome of this investigation proves that the theory works and that it is a safe and inexpensive form of technology to implement. The power emitted from the infrared LEDs was low enough to be considered safe to shine on the eye and high enough to detect changes in eye movement. A novel algorithmic solution also provided a basic classification system for the movements. However, the algorithm starts to fail as soon as more eye movements are made. The concept of reflective PPG was proven to be a viable method for measuring the heart rate non-intrusively around the ear, but the implementation of the heart rate sensor on the glasses encountered many placement and mechanical issues. By creating this pair of glasses as a development platform, this study forms the basis for further study into drowsy driving detection, heart rate monitoring, REM sleep detection, and many more. A list of future work is outlined in Section 6.2 to improve on the applications of this development system.

\subsection{Future Work}

A number of improvements can be made to the software portion of this thesis in order to make it more robust, efficient, and effective. The first improvement is to operate the Si1143 sensor in autonomous operation mode. This will make the speed of measurements not depend solely on the speed of the computer and decrease the cost of computing power when the program polls the sensor for data. This mode can also make 
use of interrupts and the internal threshold registers in the sensor to further ease computing done by the program. In order to implement the interrupts, a future software interface may be required such as programming directly onto the Arduino or some other microcontroller. Other improvements to the software could be a calibration sequence that sets the optimal current drive settings, ADC integration times of the sensor, and normalized motion for left, right, up, down, and blink movements. This improvement would then greatly increase the robustness of the classification algorithm. Additional software could also be programmed to detect the frequency of blinking and implement an alert system for a drowsy driver. The same data from the eye tracker could also be used to move a mouse on a screen for physical handicapped people or improve mobility of paraplegic patients. The data could also be used to diagnosis patients with reading disorders or diagnosis patients with REM sleep disorders.

In terms of hardware, future designs can improve upon the mechanical stability of the glasses and the convenience of it for current eyewear patients. One example could be a clip on version that attaches to the frame of the existing eyeglass wearer. It can also make use of wireless communication in order to eliminate any uncomfortable wires. Another design of the PCB glasses could also make it slimmer, incorporate actual nosepieces for comfort and stability, and adjustable temple pieces that can successfully place the sensor at a site to measure heart rate. 


\section{BIBLIOGRAPHY}

[1] D. Royal, "Volume I: Findings National Survey of Distracted and Drowsy Driving Attitudes and Behavior: 2002," The Gallop Organization, Washington, D.C., Final Report 809 566, Apr. 2003.

[2] X. Hu, R. Eberhart and B. Foresman, "Modeling Drowsy Driving Behaviors," In Proc. 2010 IEEE International Conference on Vehicular Electronics and Safety (ICVES), 2010, pp. 13-17.

[3] R. Grace, V.E. Byrne, D.M. Bierman et al. "A Drowsy Driver Detection System for Heavy Vehicles," In Proc. The AIAA/IEEE/SAE Digital Avionics Systems Conference, 1998, vol. 2, pp. I36/1-I36/8.

[4] M.J. Flores, J.M. Armingol, and A. de la Escalera, "Driver drowsiness detection system under infrared illumination for an intelligent vehicle," Intelligent Transport Systems, IET, vol.5, no.4, pp.241,251, December 2011. Available: IEEExplore, http://ieeexplore.ieee.org/stamp/stamp.jsp?tp=\&arnumber=6097595\&isnumber $=6097592$ . [Accessed May 09, 2013].

[5] M. Mehrubeoglu, L. M. Pham, H. T. Le et al. "Real-time eye tracking using a smart camera," In Proc. 2011 IEEE Applied Imagery Pattern Recognition Workshop (AIPR), 2011, pp.1,7, 11-13.

[6] Medical Eye Services Vision Administration, "Vision Facts and Statistics," Medical Eye Services Vision Administration, 2005. [Online]. Available:

https://www.mesvision.com/includes/pdf_Broker/MESVision\%20Facts\%20and\%20Statis tics.pdf [Accessed May 09, 2013].

[7] M. Yaso, A. Nuruki, S. Tsujimura et al. "Detection of REM sleep by heart rate," In Proc. The First International Workshop on Kansei.

[8] A. Iranzo, J. L. Molinuevo, J. Santamaría et al. "Rapid-eye-movement sleep behaviour disorder as an early marker for a neurodegenerative disorder: a descriptive study," The Lancet Neurology, vol. 5, no. 7, pp. 572-577, July 2006. Available: ScienceDirect, http://www.sciencedirect.com/science/article/pii/S1474442206704768. [Accessed May 09, 2013].

[9] Silicon Laboratories, "Proximity/Ambient Light Sensor IC with $\mathrm{I}^{2} \mathrm{C}$ Interface," Si1141/42/43 datasheet, Jan. 2011 [Revised May 2012].

[10] Silicon Laboratories, Appl. Note 521. 
[11] CENELEC, "Photobiological safety of lamps and lamp systems," IEC-62471, 01 Sept., 2008

[12] Silicon Laboratories, Appl. Note 498.

[13] OSRAM Opto Semiconductors, "High Power Infrared Emitter (850 nm)," SFH 4056 datasheet, [Revised Nov. 2010].

[14] OSRAM Opto Semiconductors, Appl. Note Eye Safety of IREDs used in Lamp Applications.

[15] L. Wang, B. Lo, and G. Yang, "Reflective photoplethysmograph earpiece sensor for ubiquitous heart rate monitoring," In Proc. 4th Int. Workshop Wearable Implantable Body Sens. Netw. (BSN2007), ser. IFMBE Proc., S. Leonhardt, T. Falck, and P. M“ah"onen, Eds., Aachen, Germany, Mar. 2007, vol. 13, pp. 189-194

[16] M. Häggström, "Superficial temporal artery,” Image, Wikimedia Commons, October, 6, 2007. [Online]. Available:

http://commons.wikimedia.org/wiki/File:Superficial_temporal_artery.PNG\#. [Accessed June 11, 2013]. 


\section{APPENDIX A: KEIL QUOTATION}

\section{Quotation}

Keil, an ARM Company

150 Rose Orchard Way

San Jose, CA 95134-1358

Phone: 972-312-1107

FAX: $\quad 972-312-1159$

To: RICKWONG

CALPOLY

190 CALIFORNIA BLVD APT 304

SAN LUIS OBISPO, CA 93405

USA

Phone: 415-370-2335

FAX:

\section{Quantity Part Number Description}

$1 \quad$ CA5 1

CA51 COMPILER KIT

Payment Method:

Prepaid (remit payment to the above address)

- PO Number:

*A copy of the $\mathrm{PO}$ document is required.

Credit Card:

Please Use CC Form
Quote Number Expiration Date

$$
58984 \quad 2 / 29 / 2012
$$

Representative

LABRE

Signature:

Print Name:

- All prices quoted are in US Dollars and are good in the US only.

- Orders are shipped vis UPS Blue (two day), unless specified otherwise.

- Orders are shipped vis UPS Blue (two day), unless specified o

- Purchase orders cannot be acoepted for orders under $\$ 1000$.

- Tax, Shipping and Handling are NOT included in this quote.

- All information subject to Keil's terms and policy. For more details see the Price List or contact your Account

Manager.

- This quote has been provided to you as a convenience only. Pricing is subject to change and is not

guaranteed. Keil, an ARM Company reserves the right to modify or withdraw this quote at any time. 


\section{APPENDIX B: LABVIEW CODE}

The code for the final version of the right side of the glasses is shown below. First the front panel is shown overall with zoomed in pictures following it. The block diagram follows the same format. All Sub VIs of the code are shown with the front panel next to the block diagram.

Thesis_right_side_v6.vi Front Panel
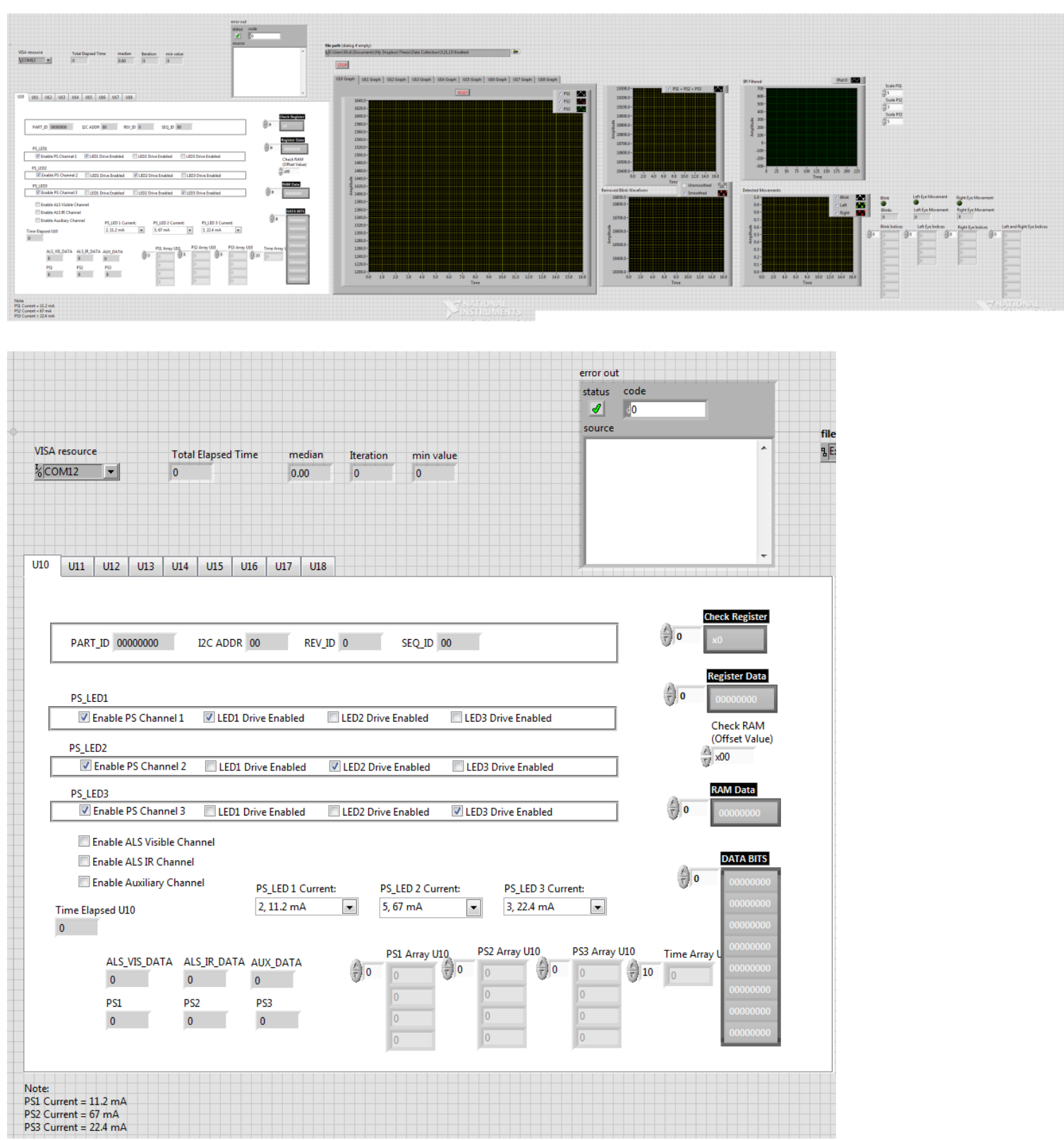


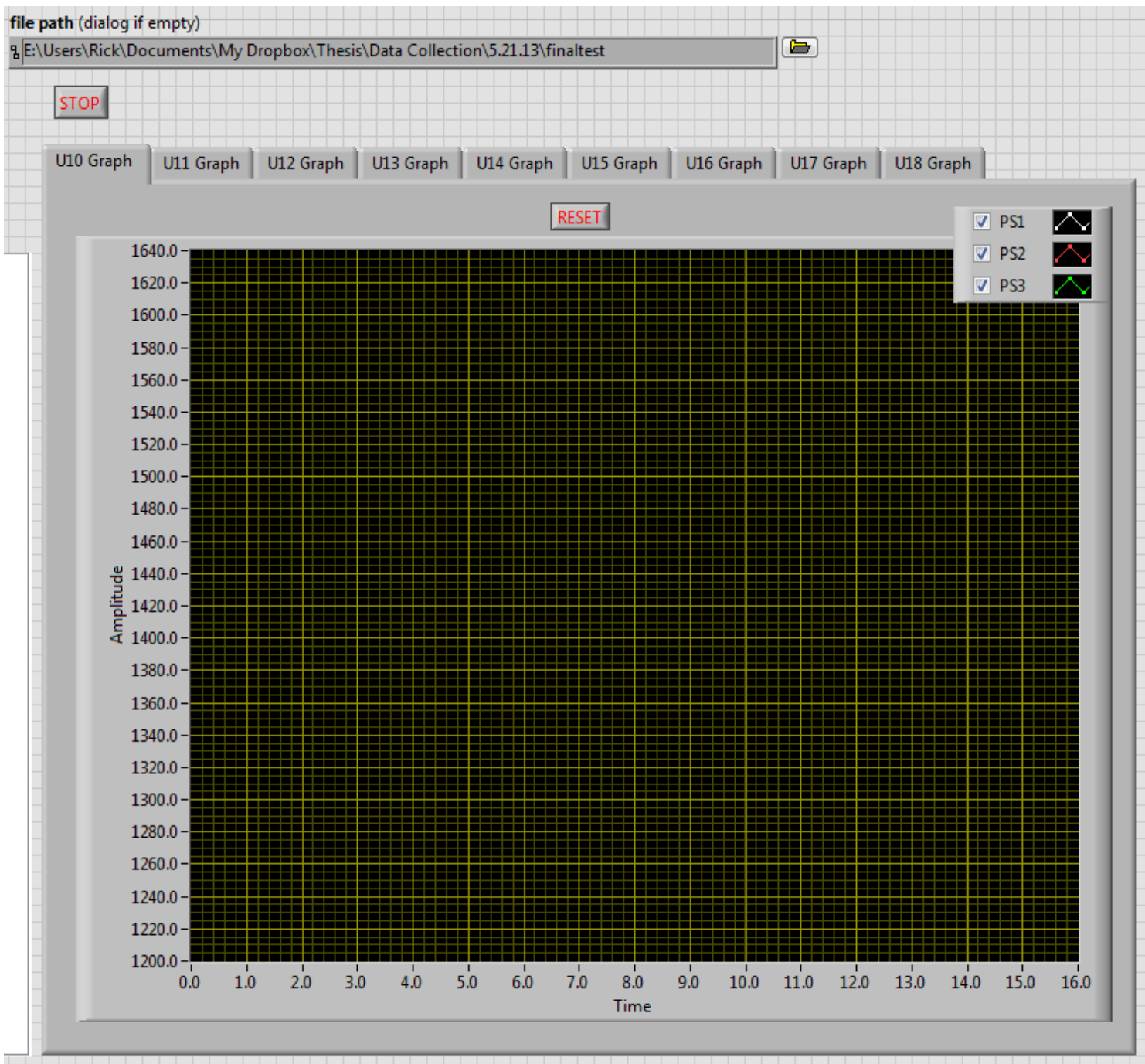



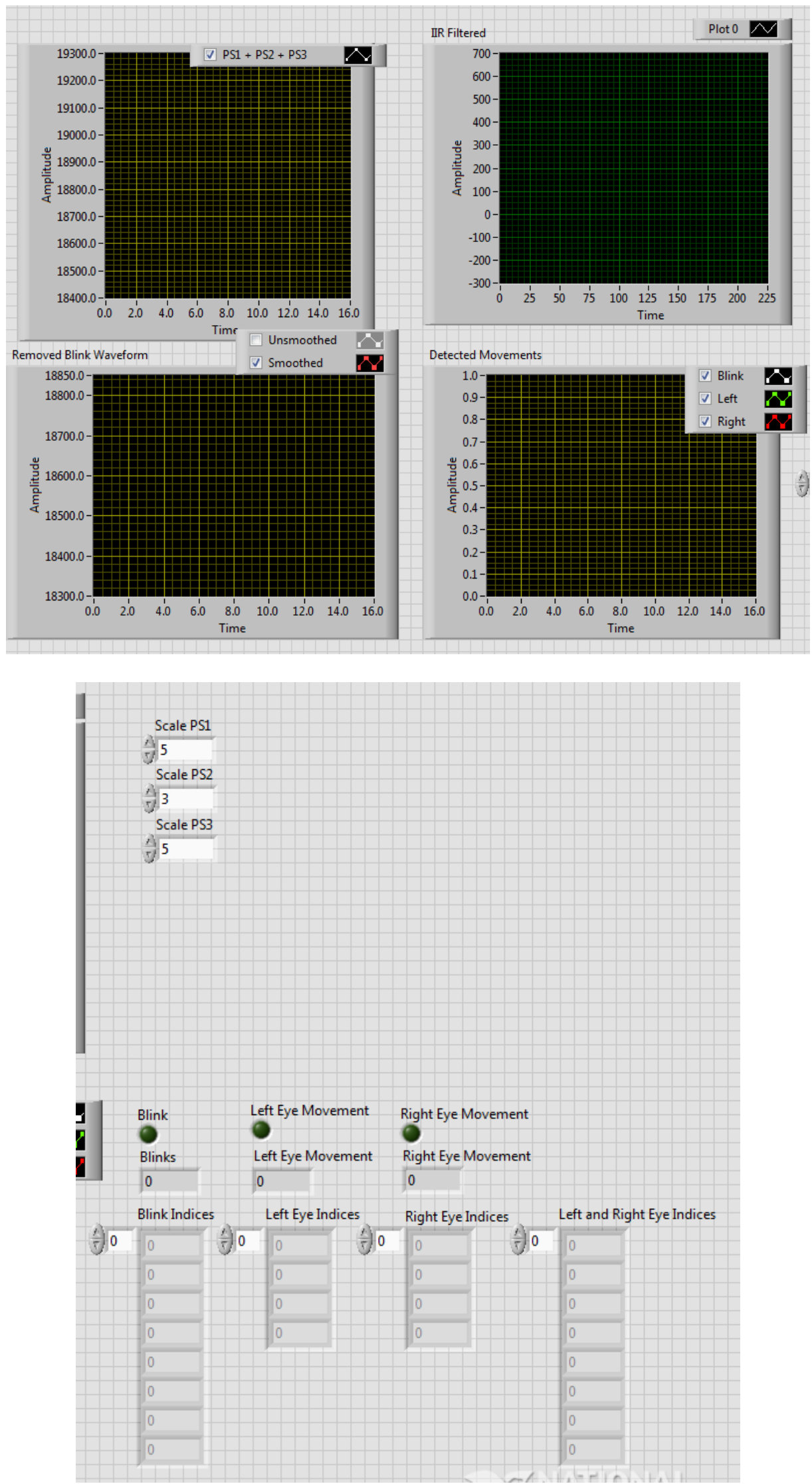
thesis_right_side_v6.vi Block Diagram
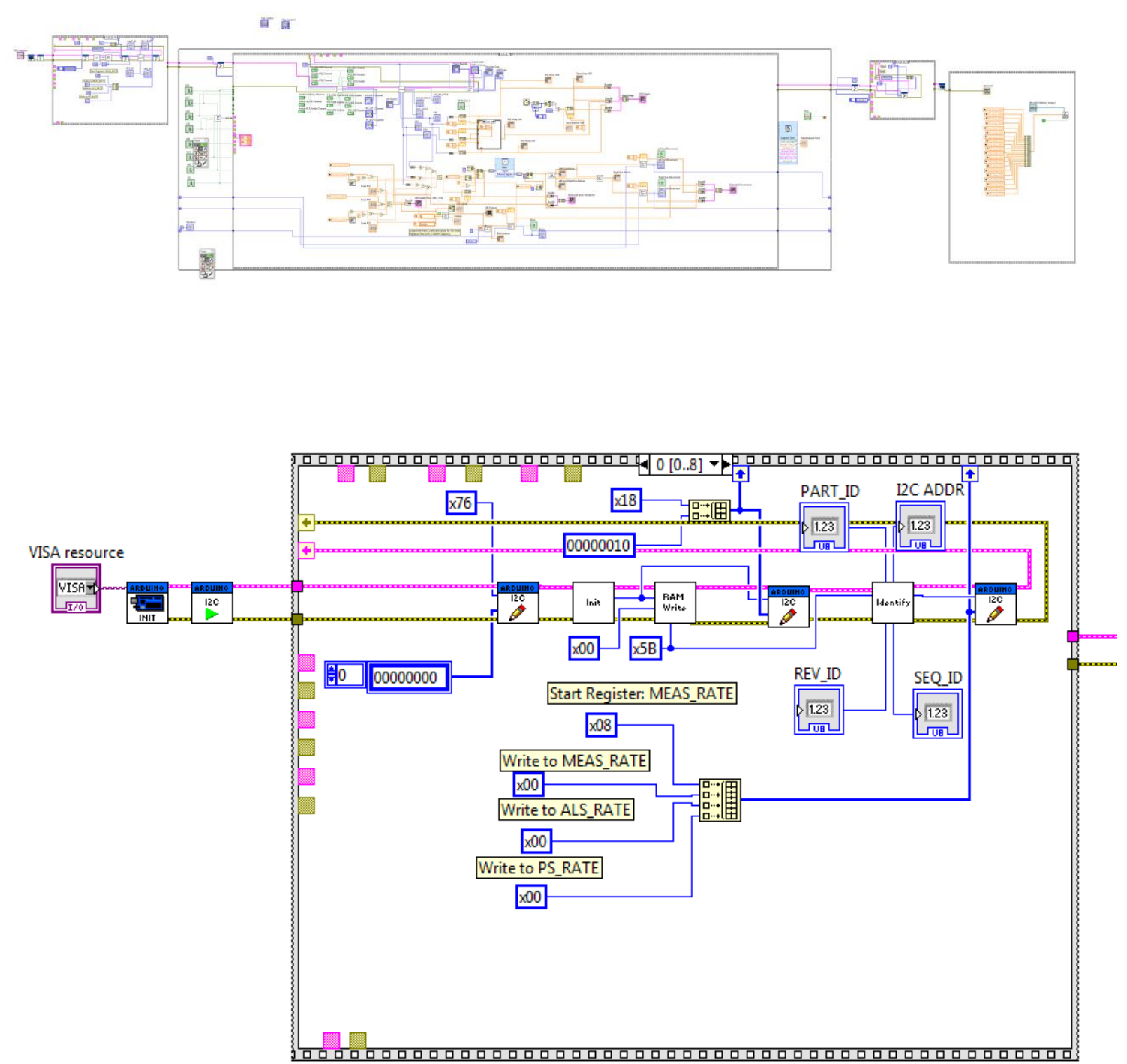

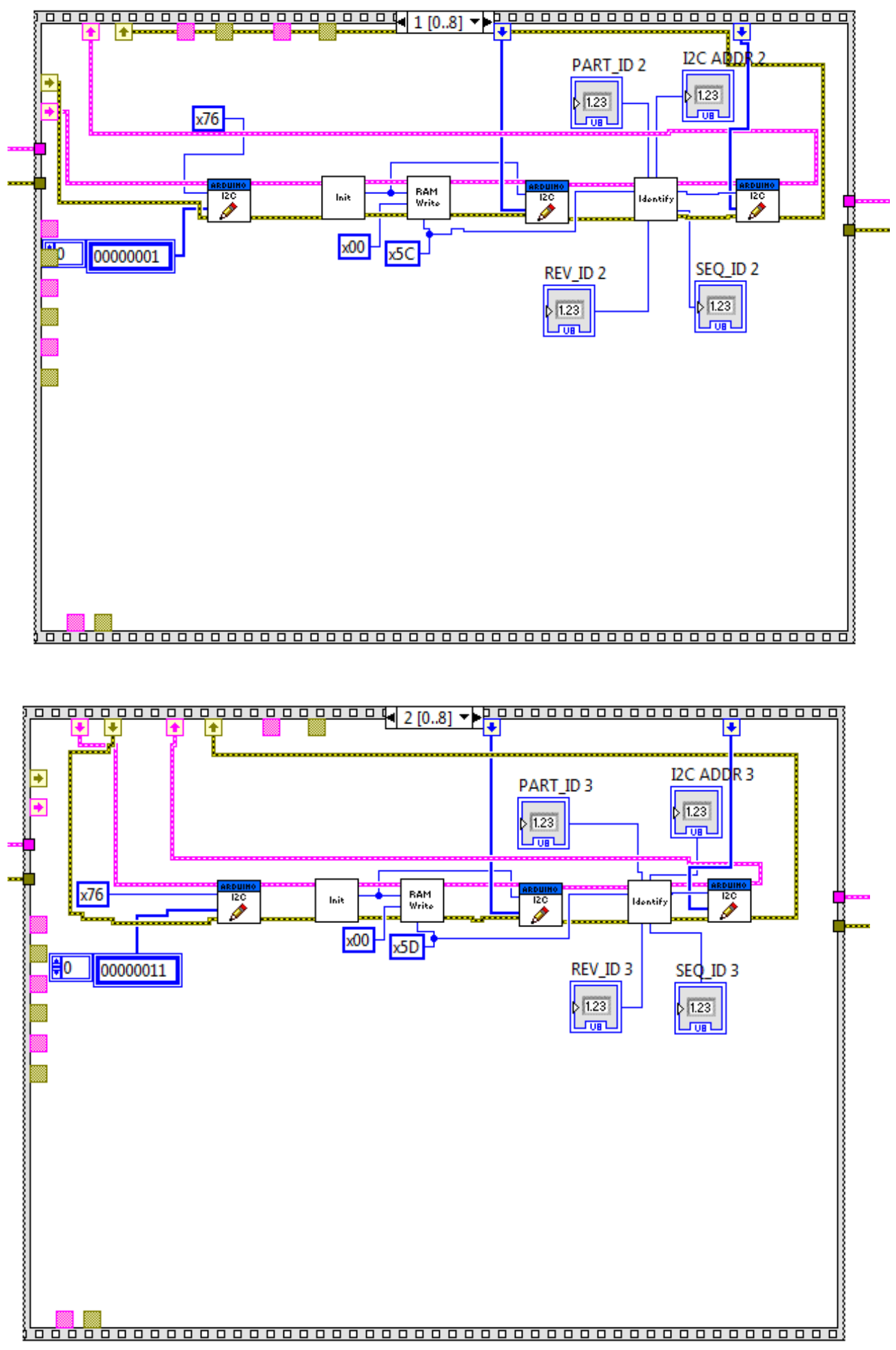

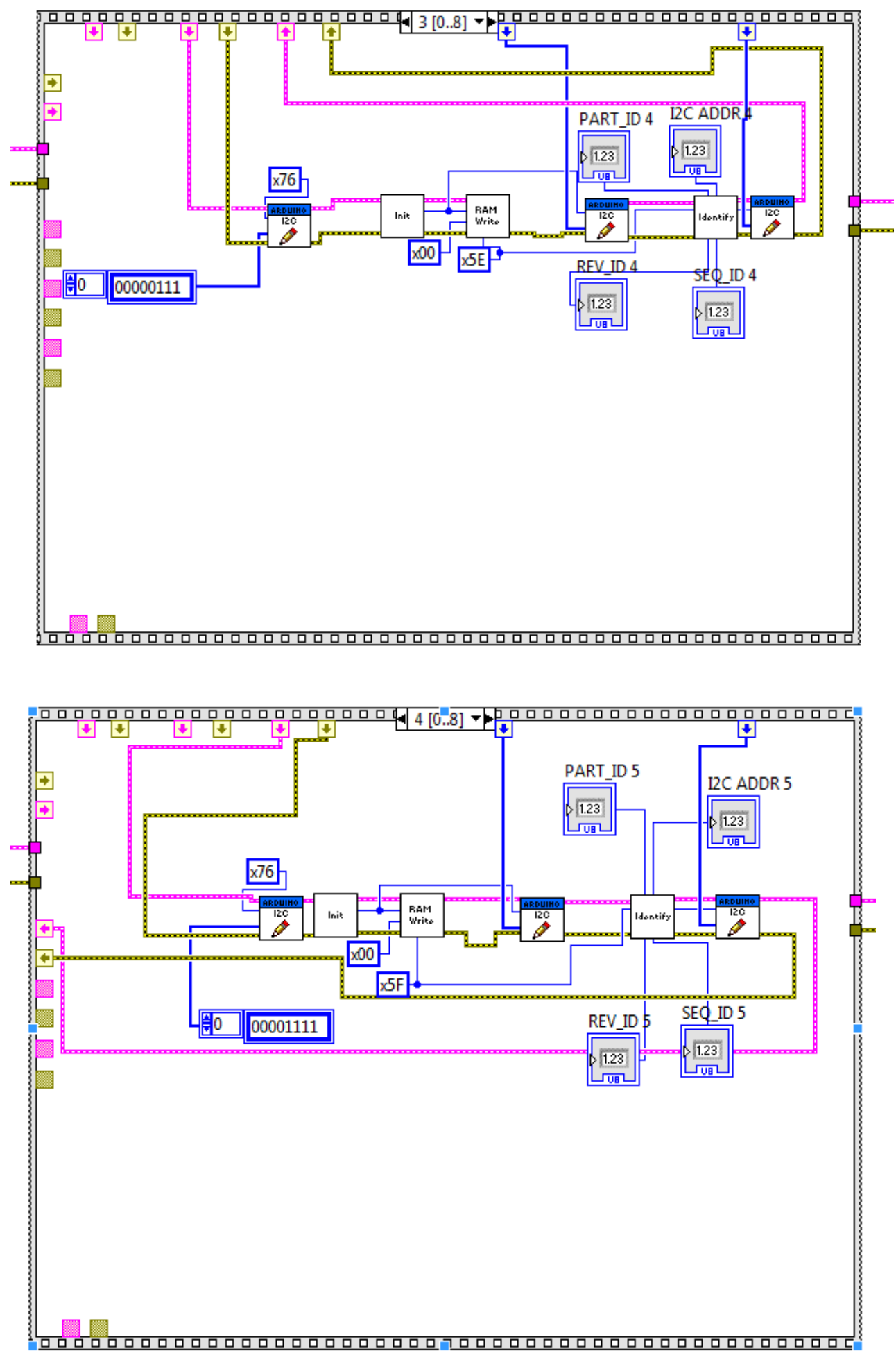

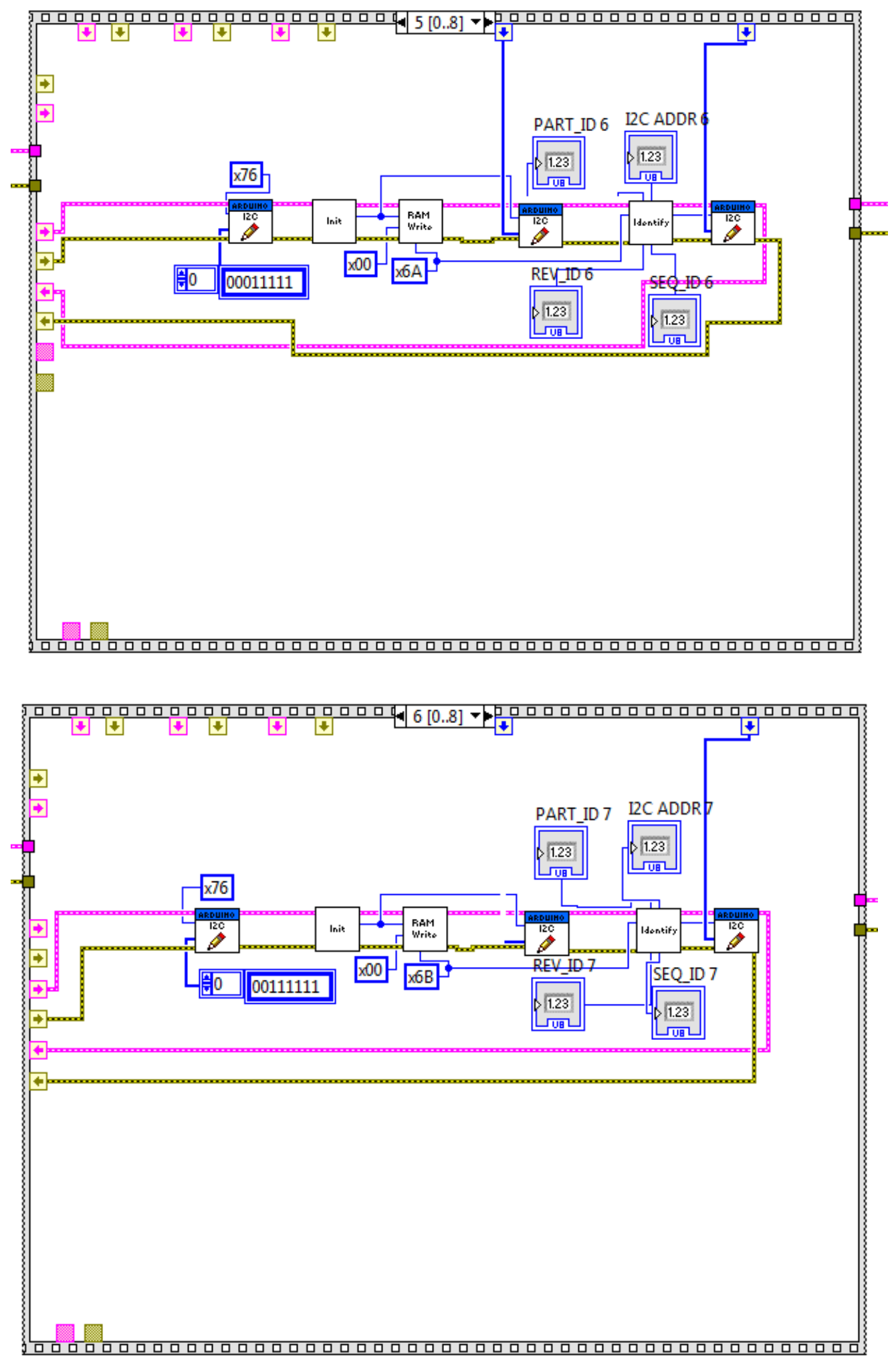

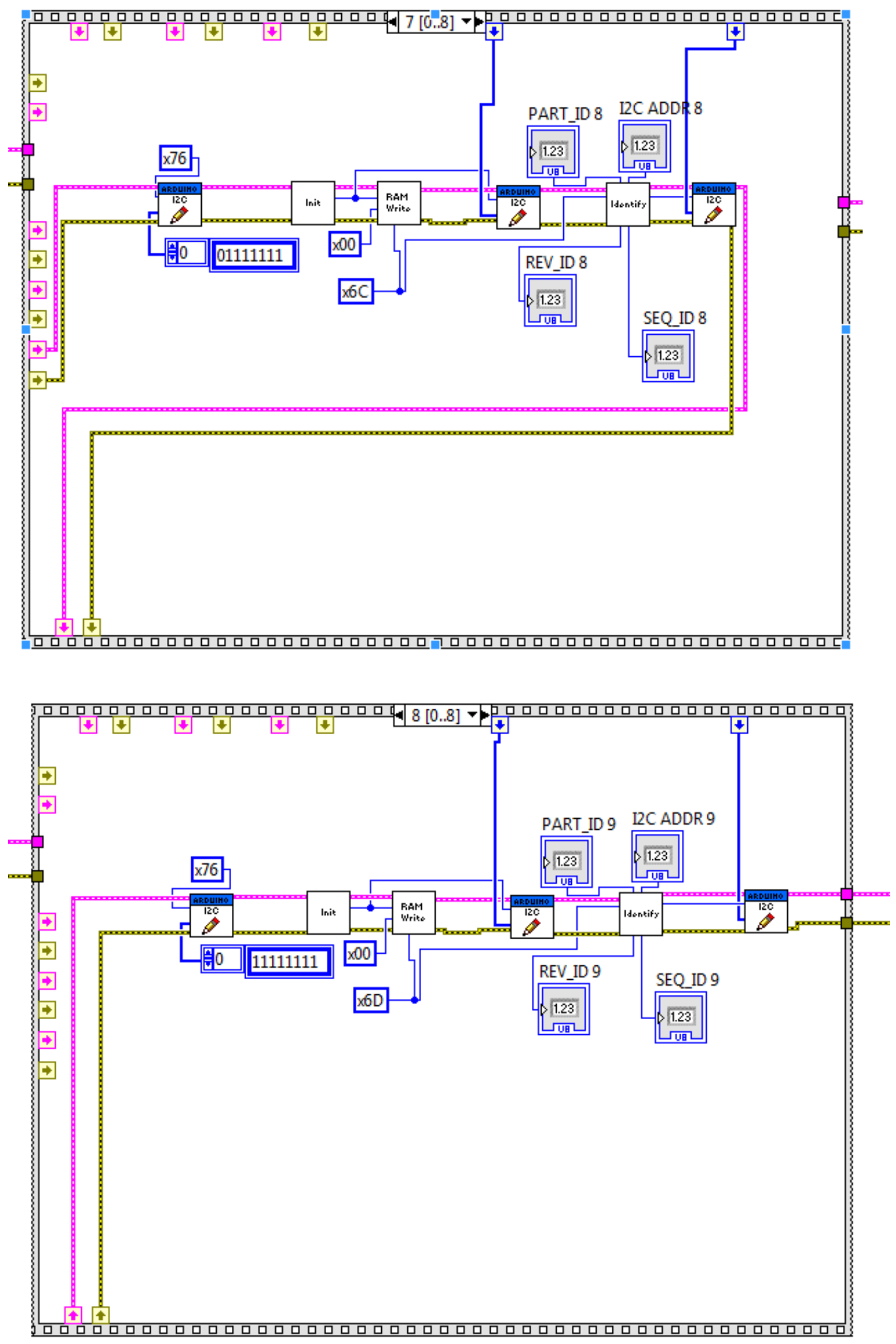

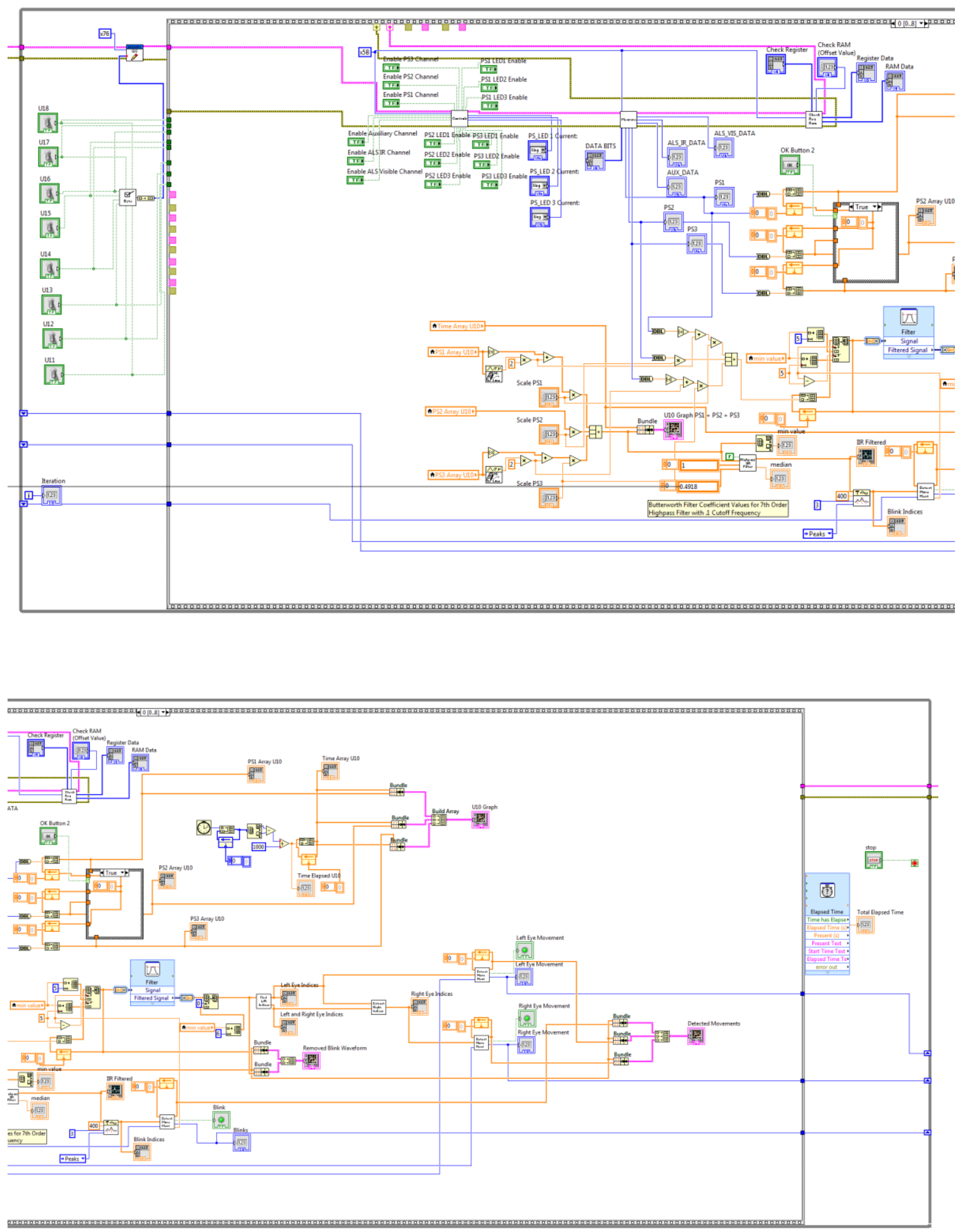


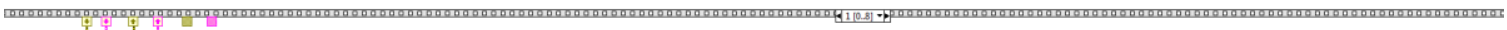

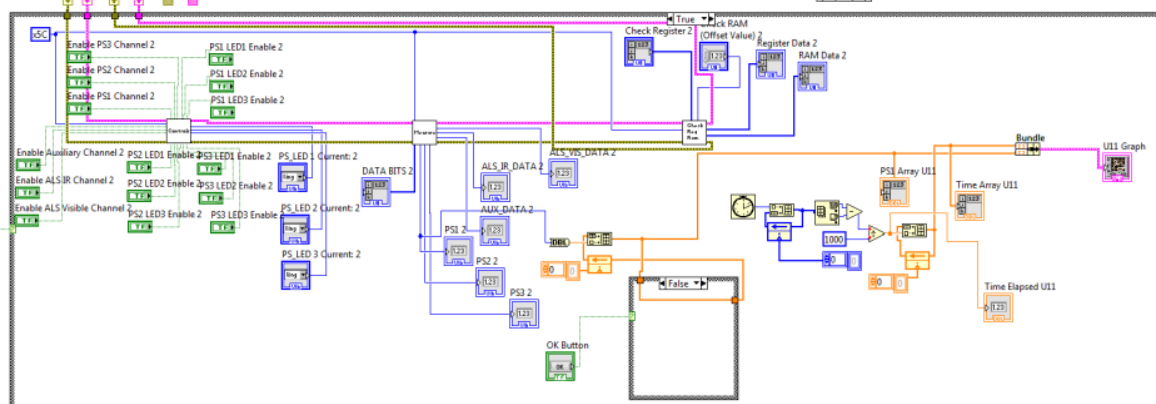

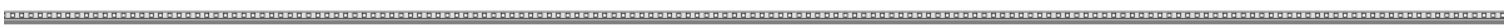
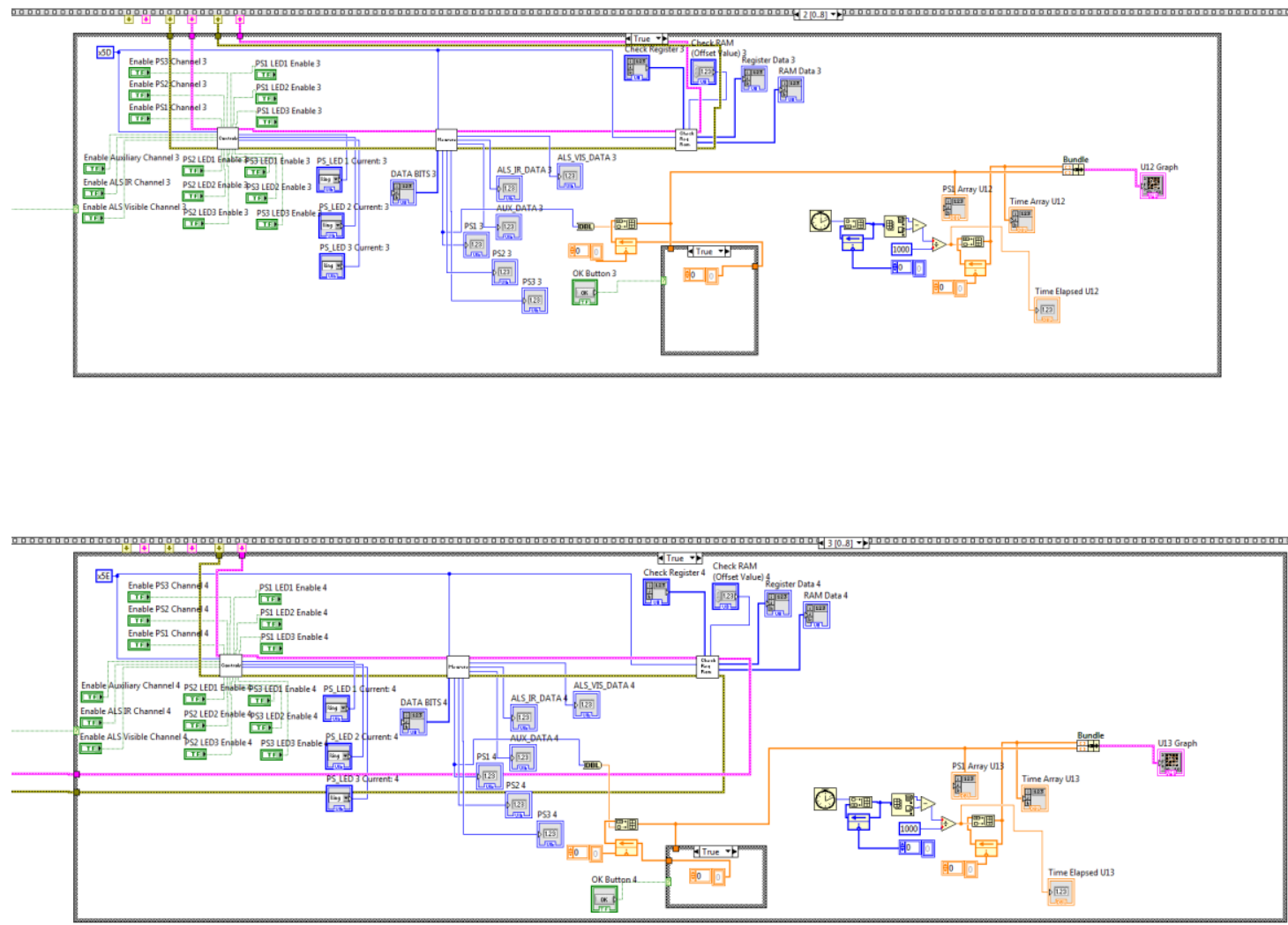

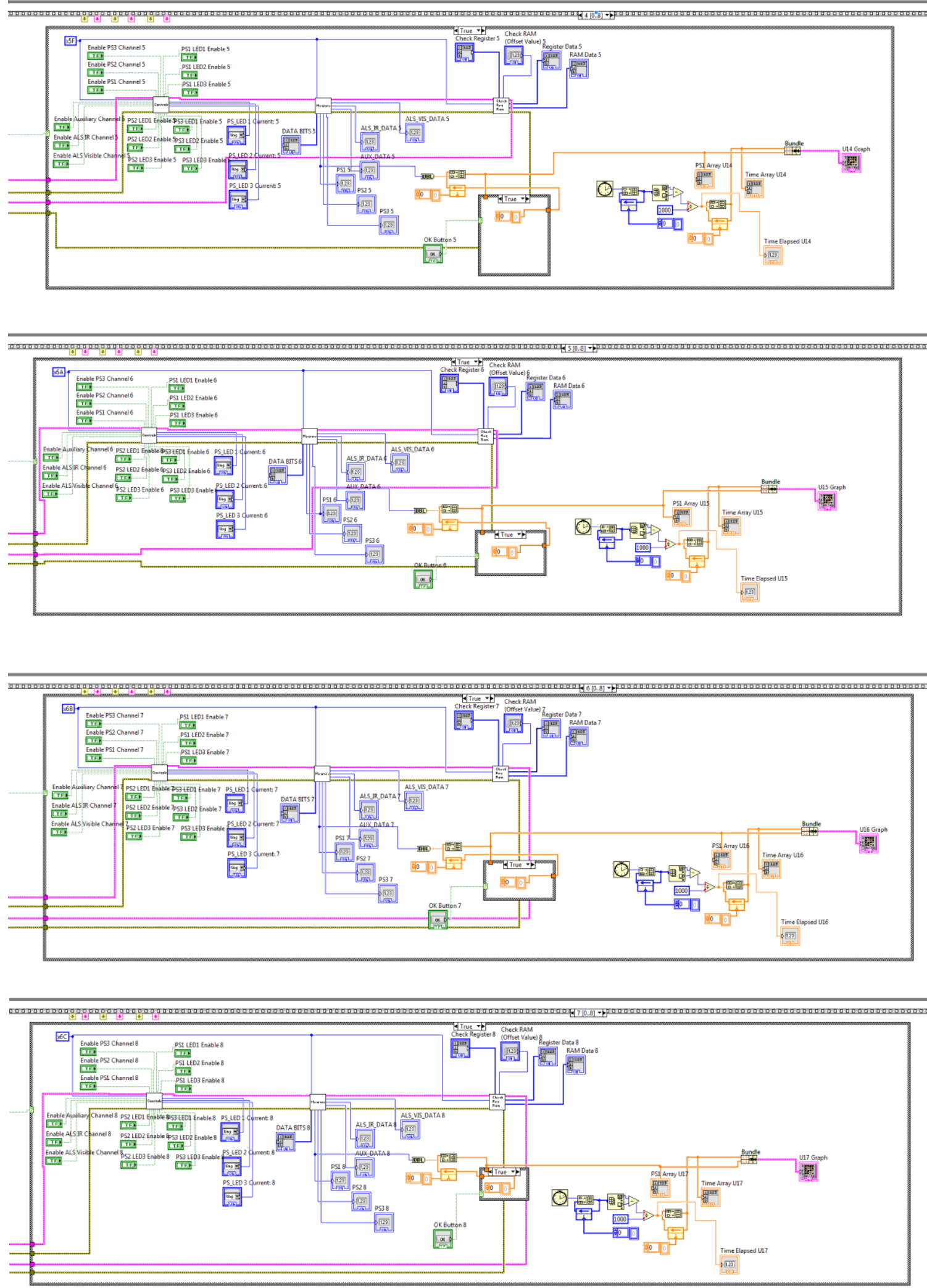

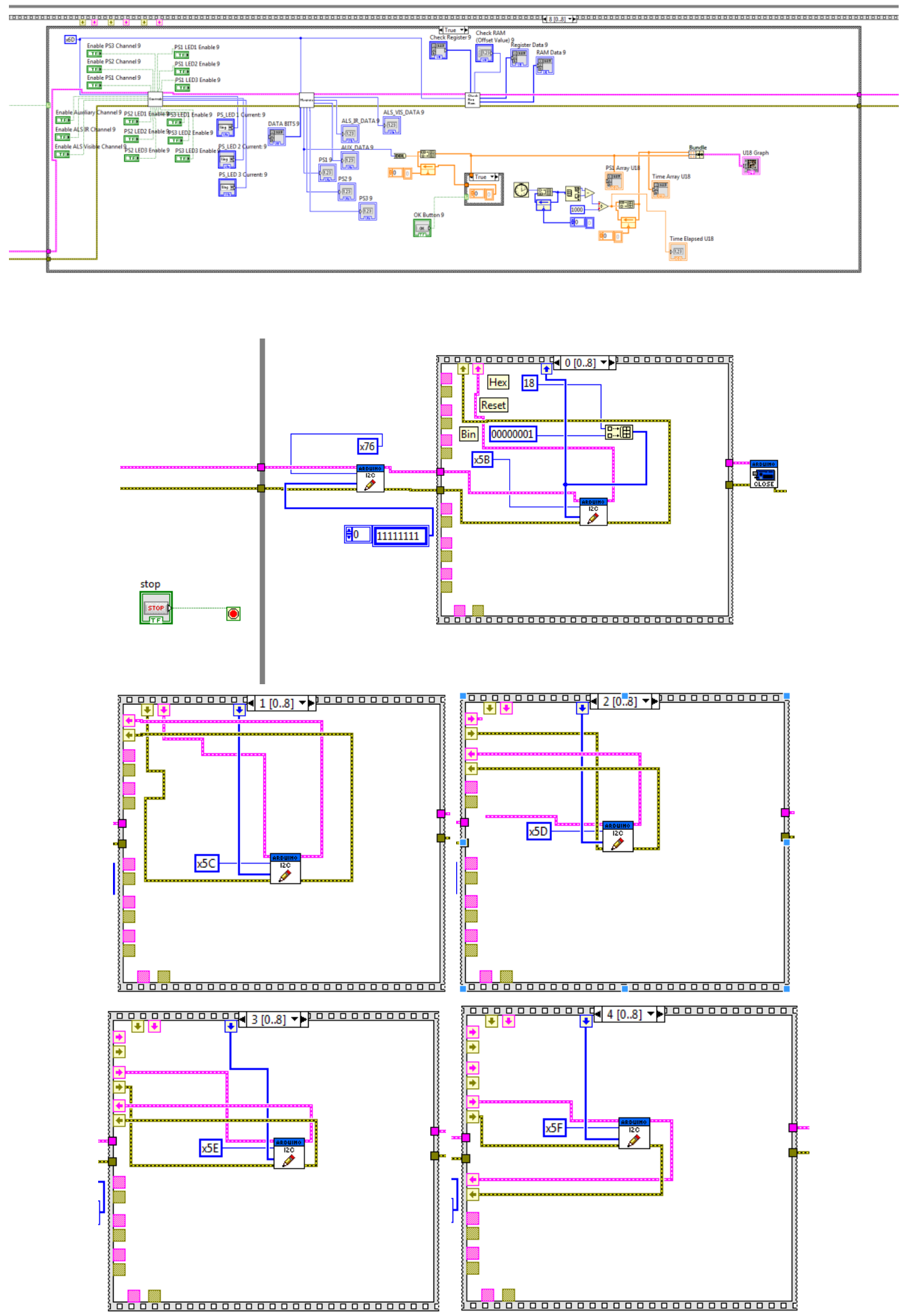

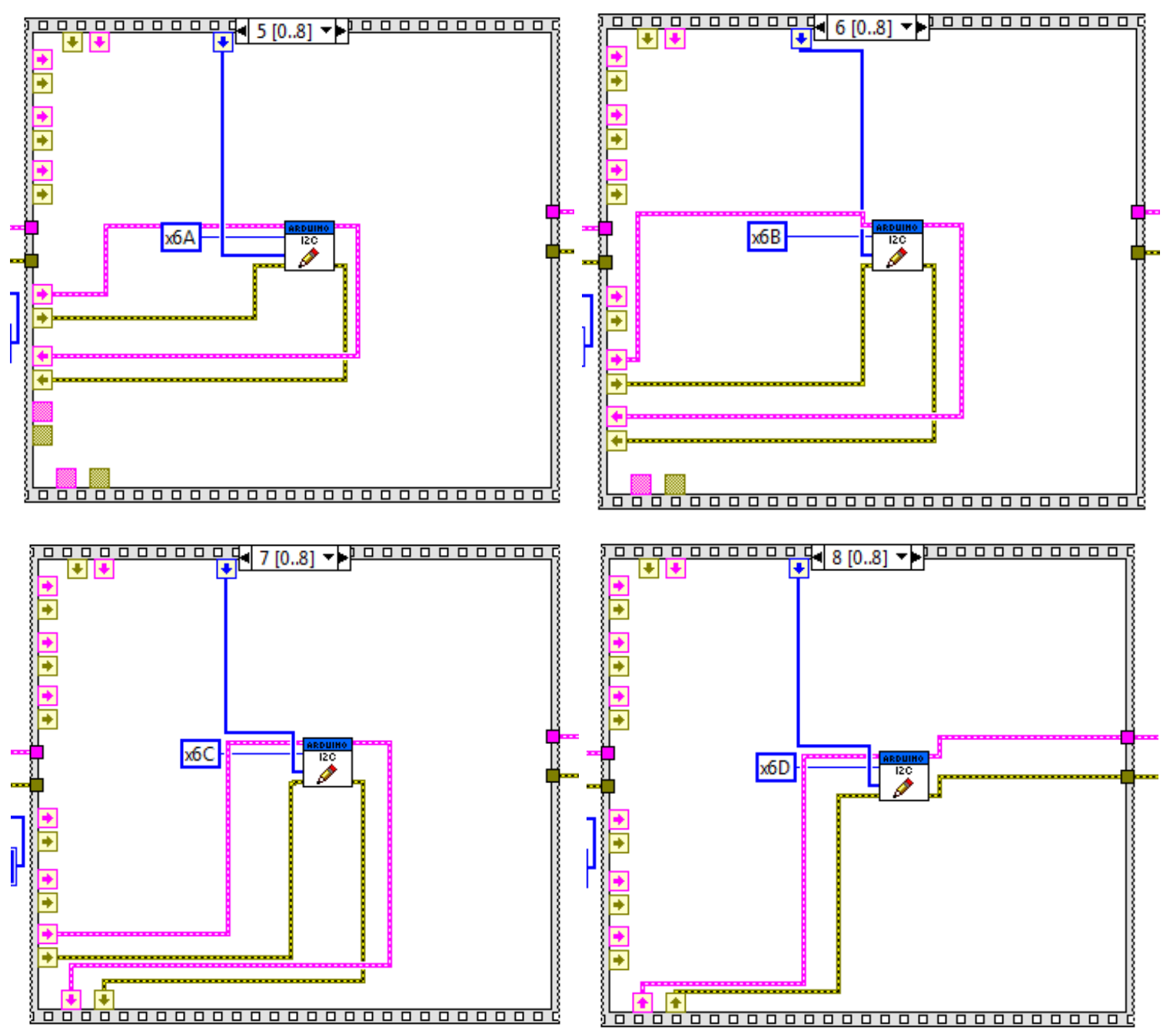


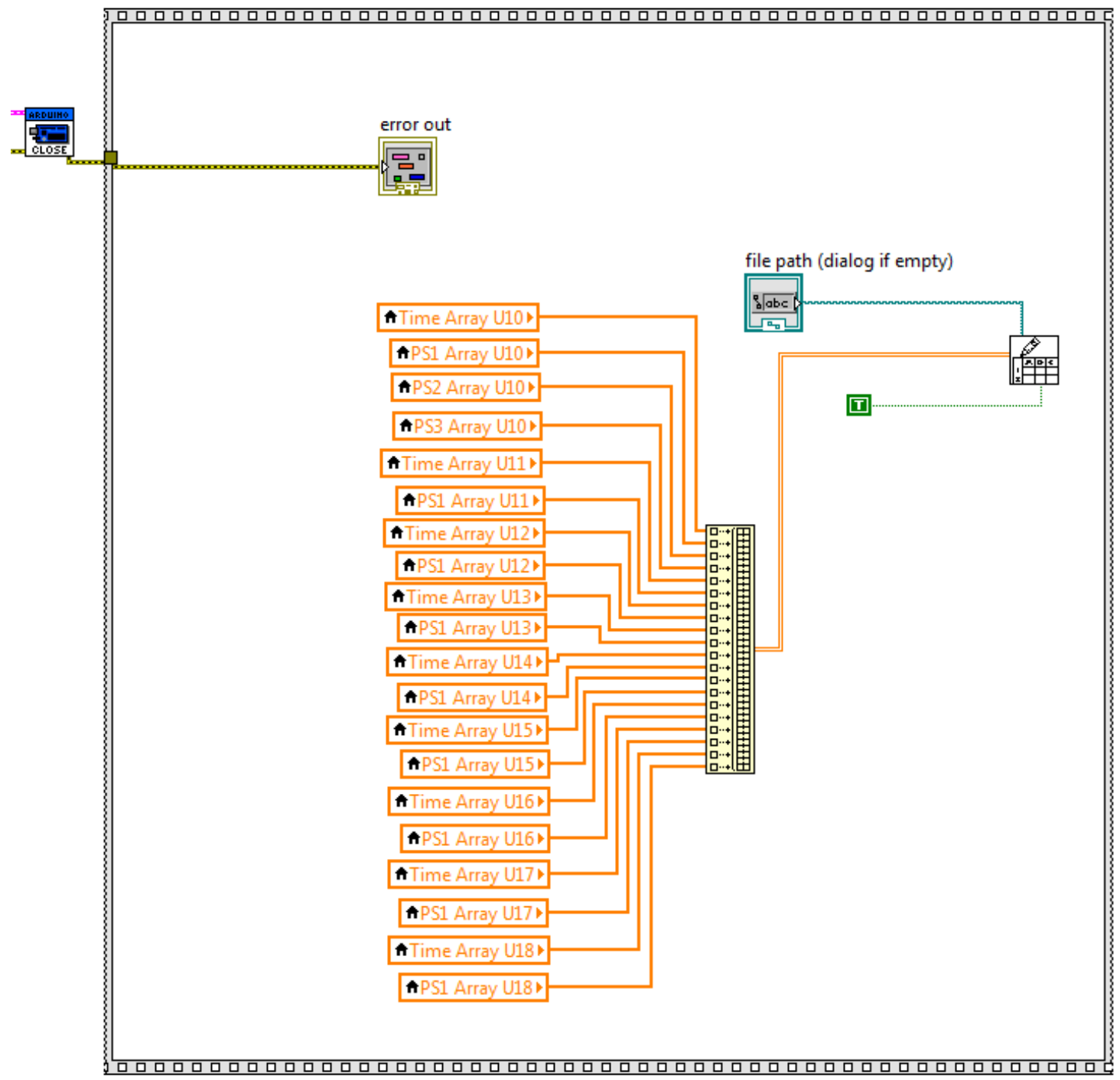




\section{Init_Si1143 (SubVI).vi}
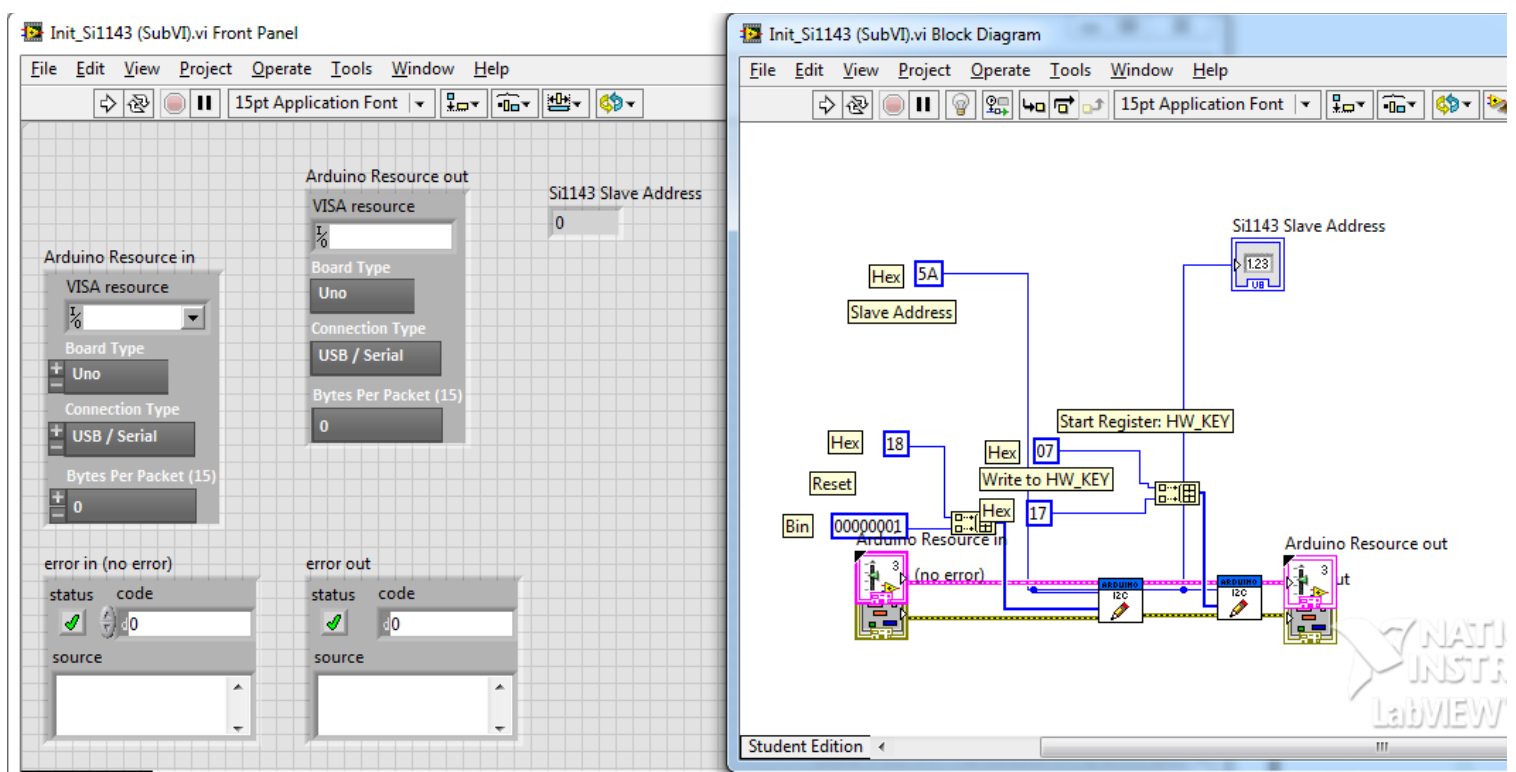

\section{$\underline{\text { RAM write (SubVI).vi }}$}
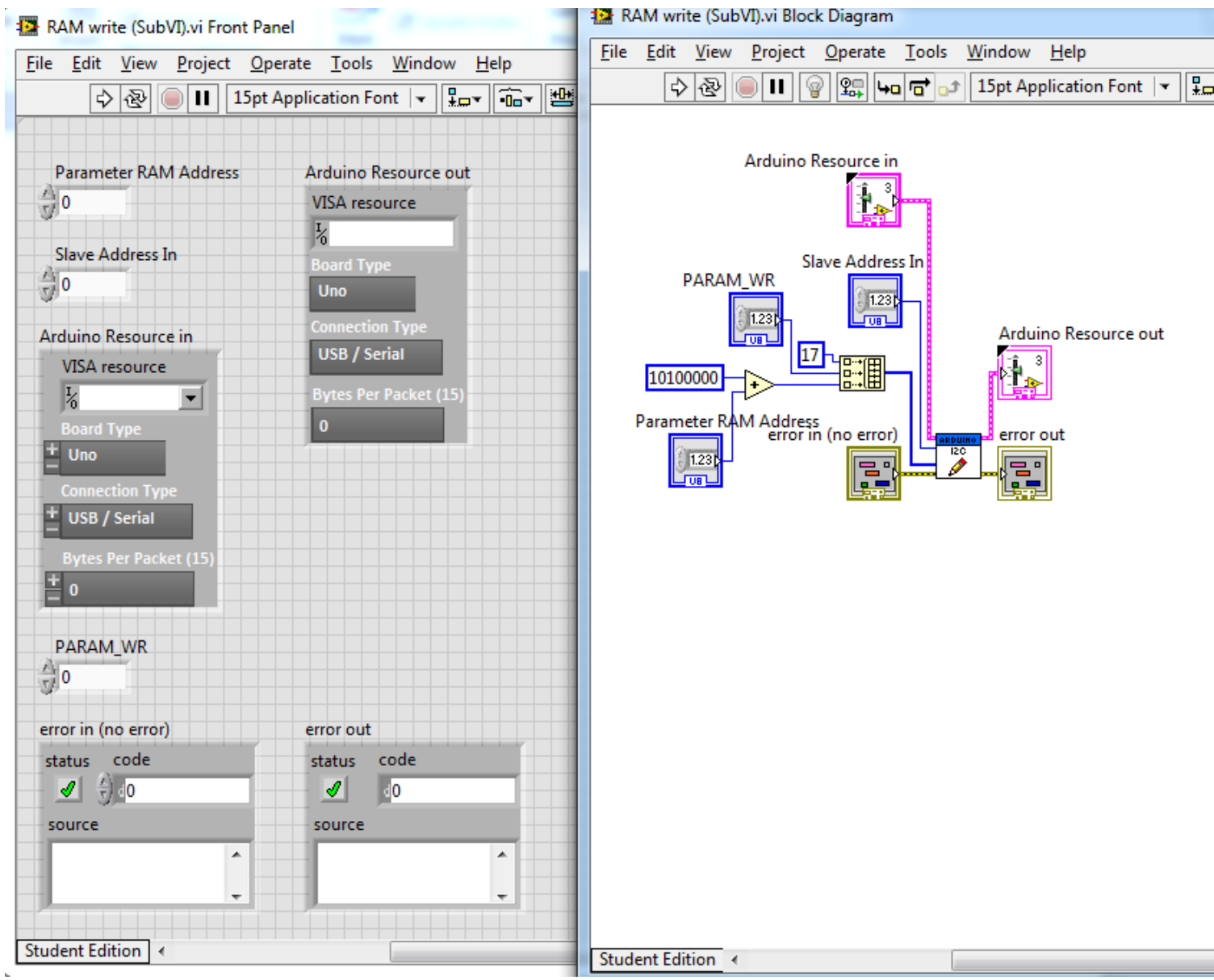


\section{Identify_Si1143 (SubVI).vi}

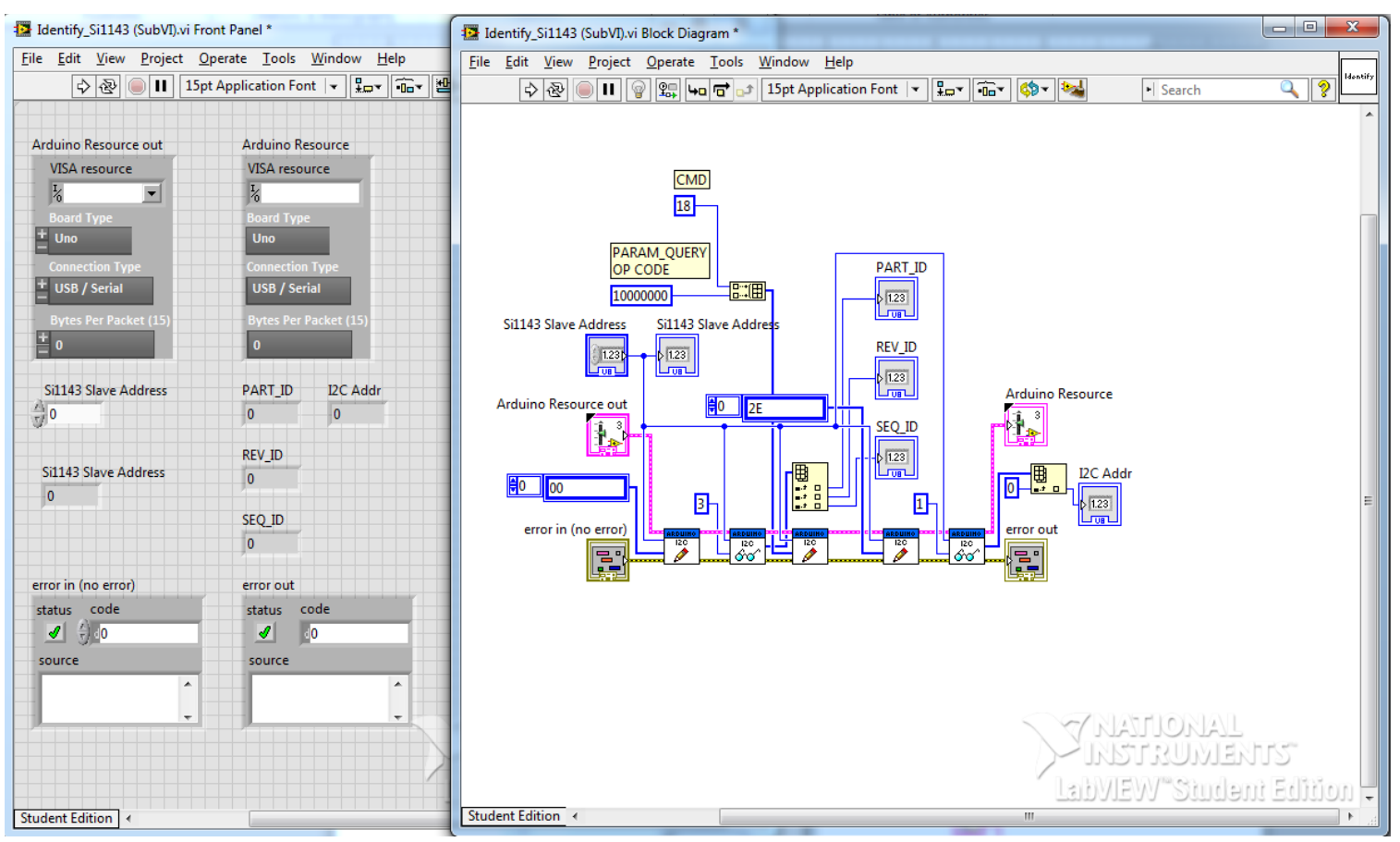

\section{Checkbox to Byte (SubVI).vi}

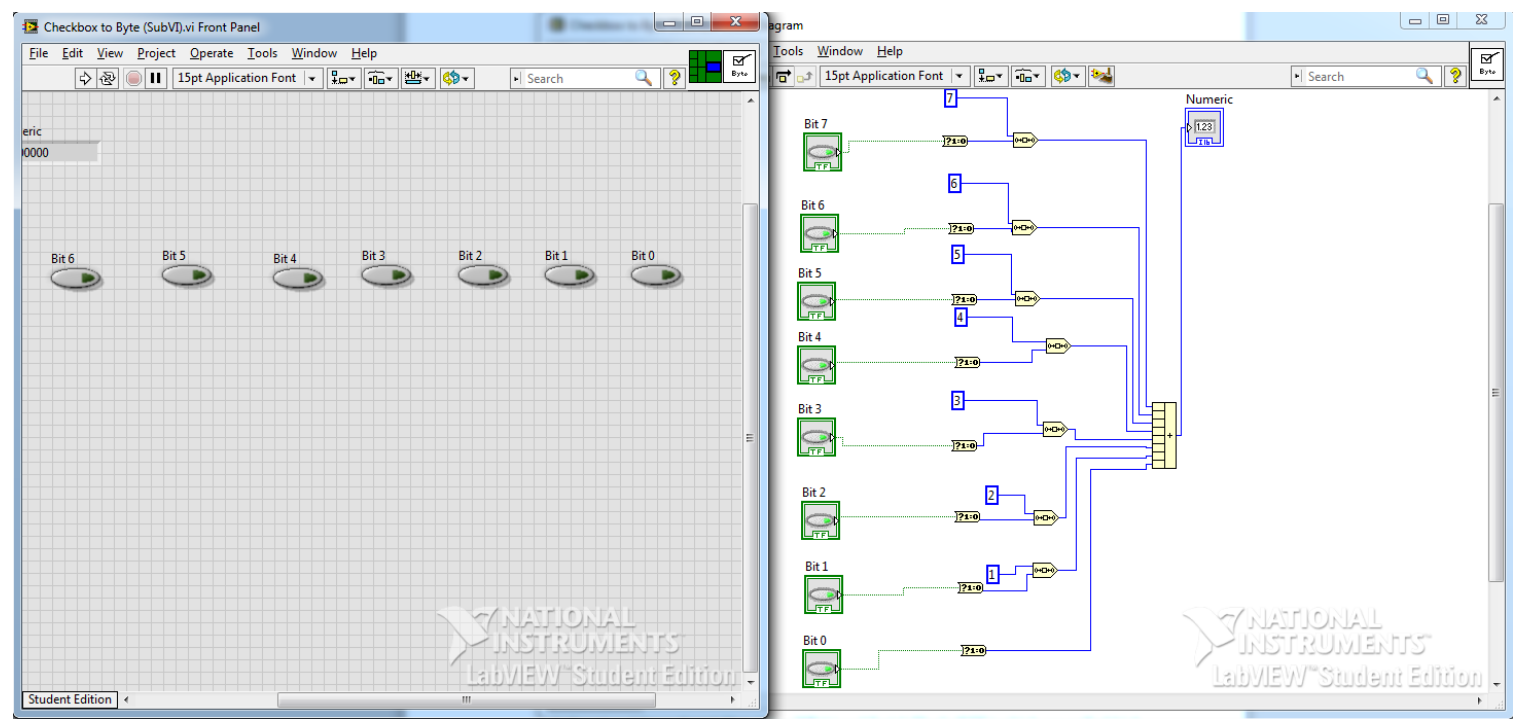




\section{Controls (SubVI).vi}

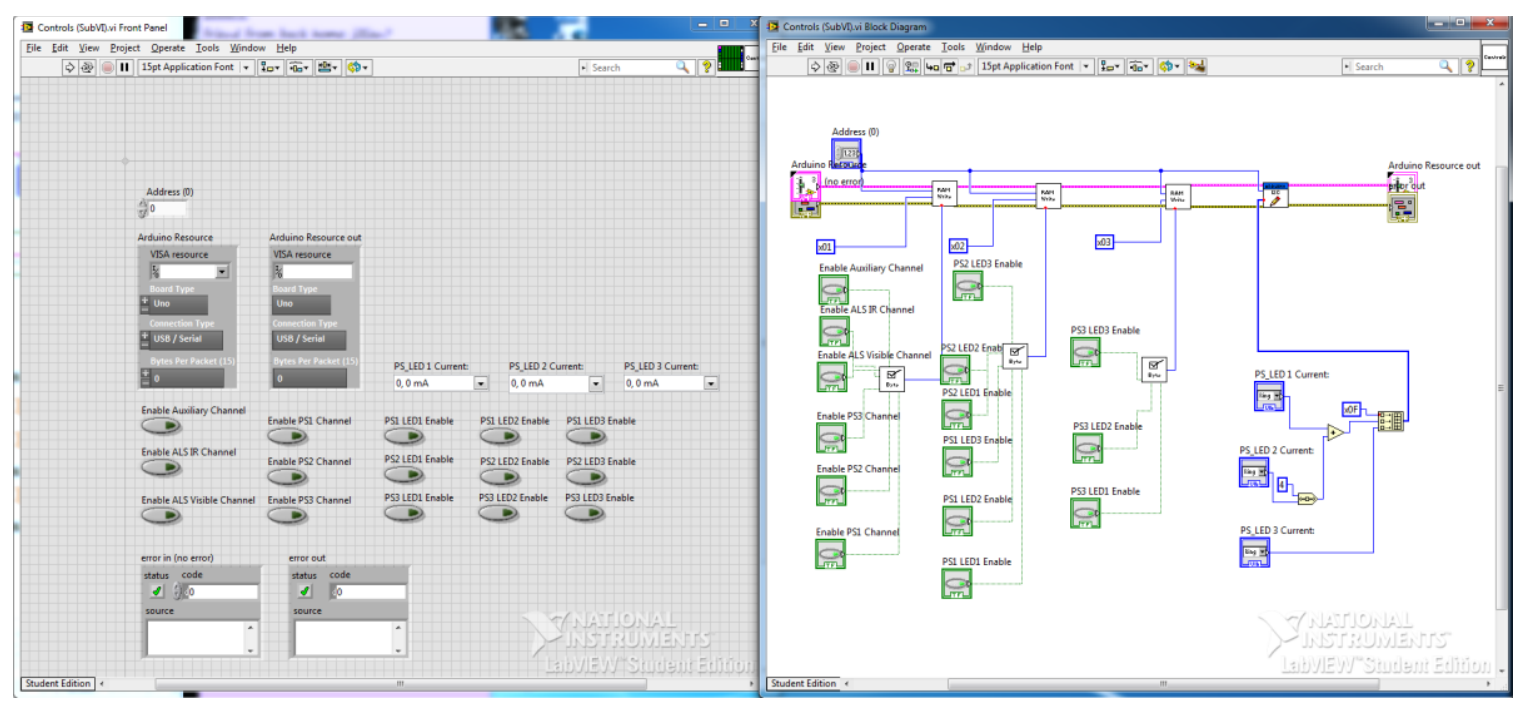

\section{Measure (SubVI).vi}

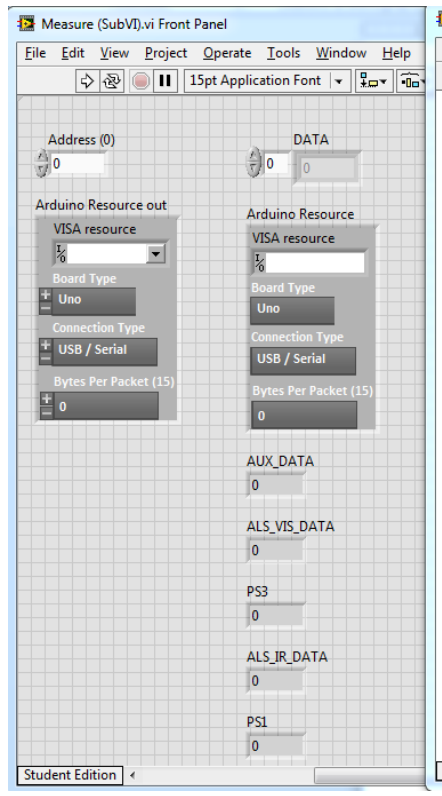

7 Measure (SubVI) vi Block Diggram Eile É $\underline{E}_{\text {dit }}$ View $\underline{\text { Project }} \underline{\text { Operate }}$ Iools Window Help

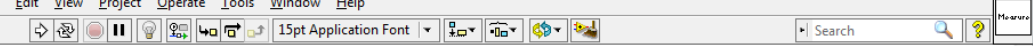
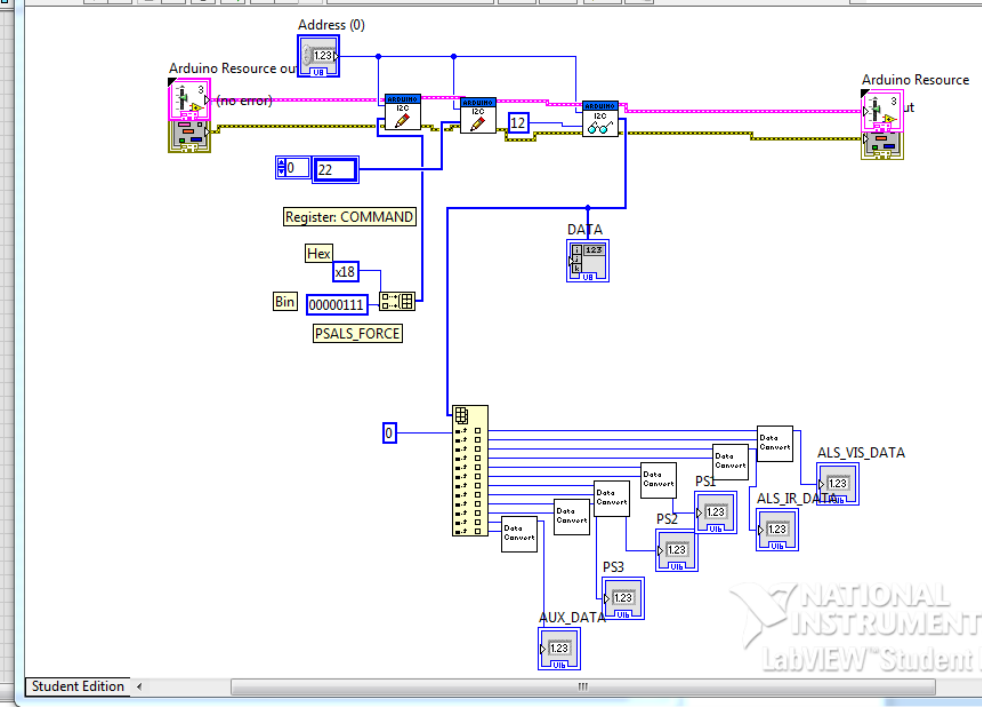

$\mathrm{Hex}$

Bin 00000111 _圆: PSALSFORCE

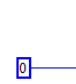

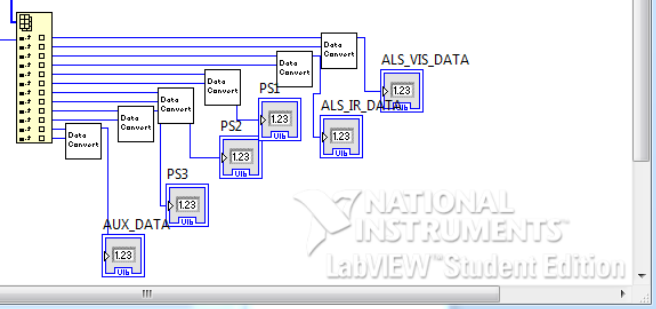

\section{Uncompression Conversion (SubVI).vi}

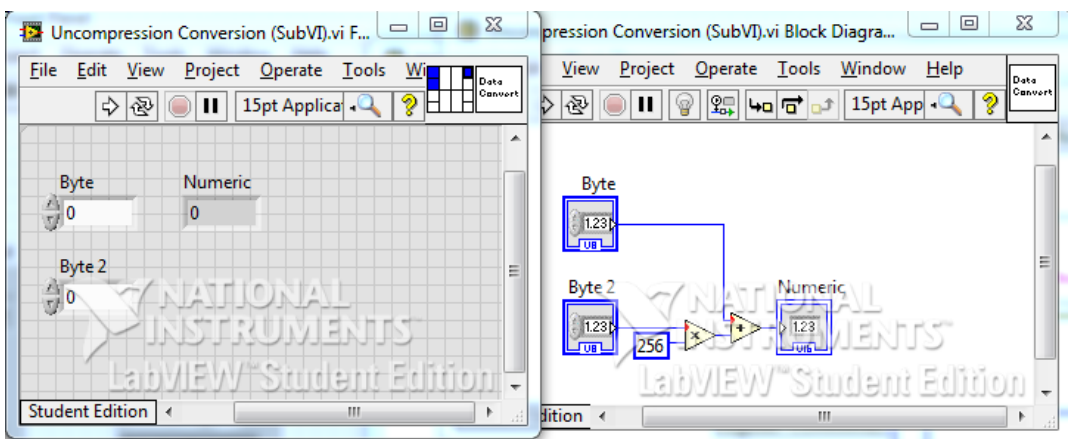




\section{CheckRegRAM (SubVI).vi}

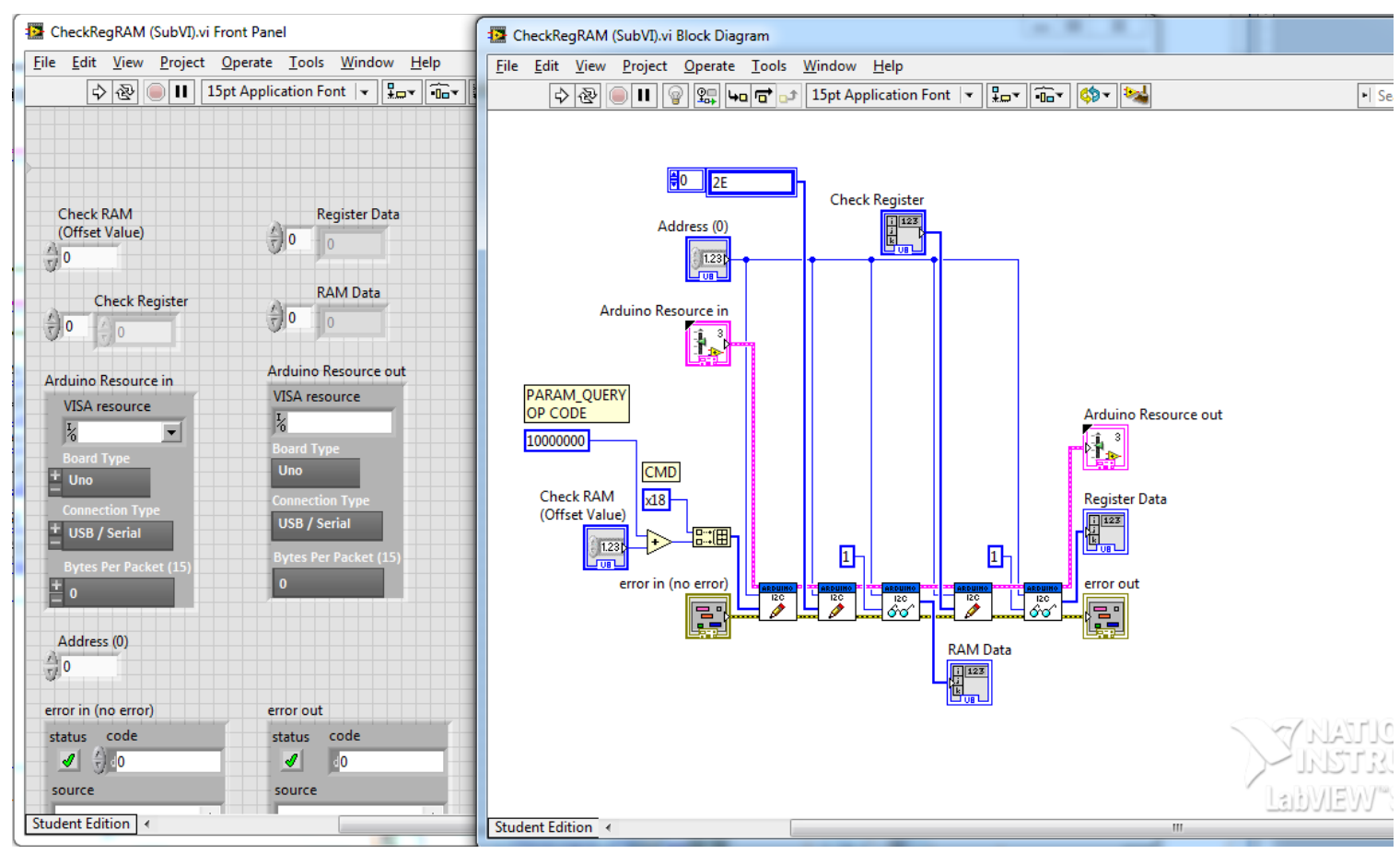

\section{IIR Filter (SubVI).vi}
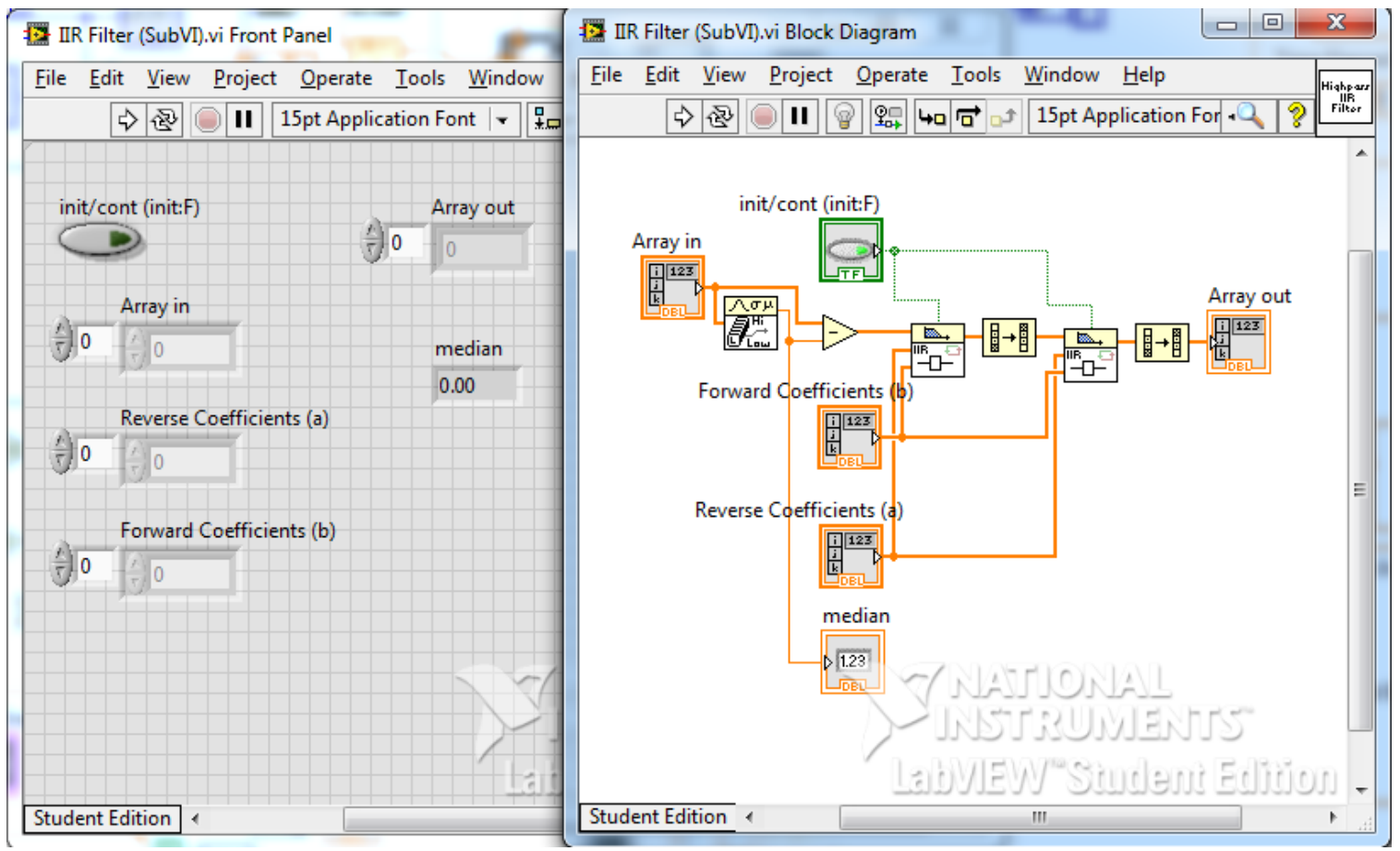


\section{Detect Movement (SubVI).vi}

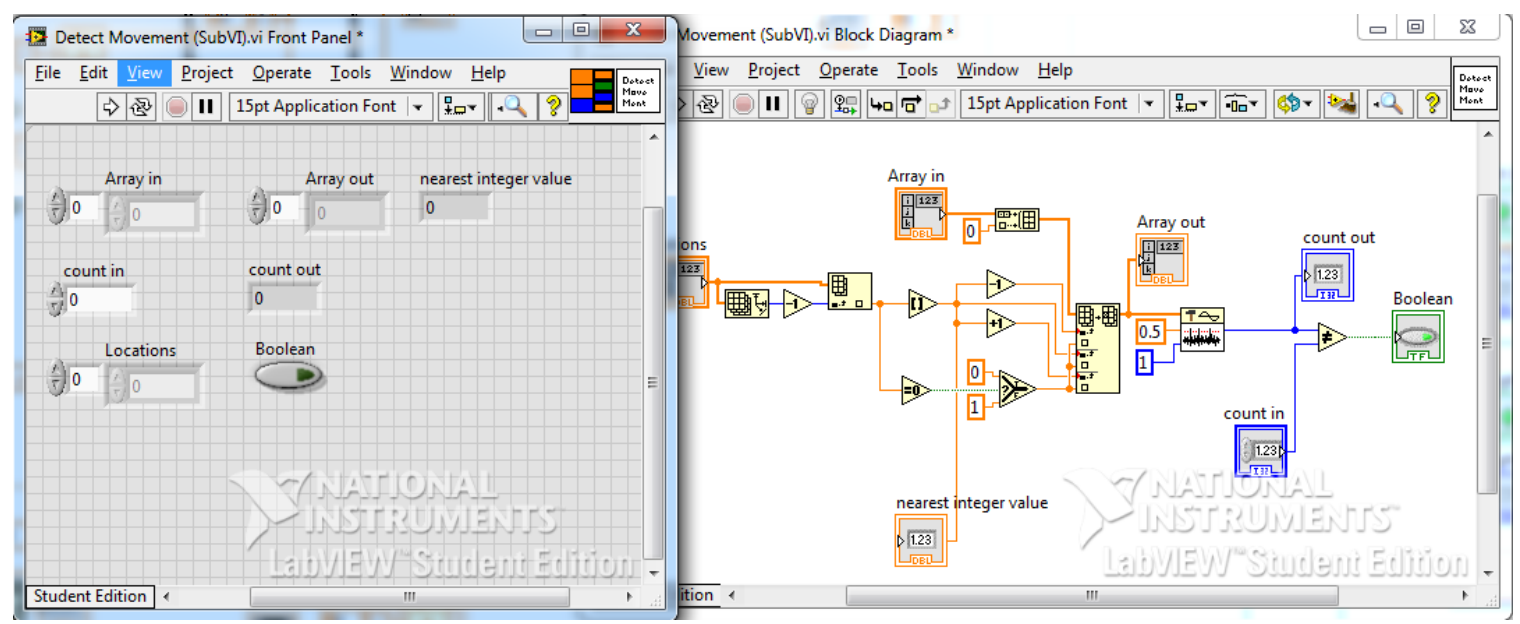

\section{Find LR Indices (SubVI).vi}

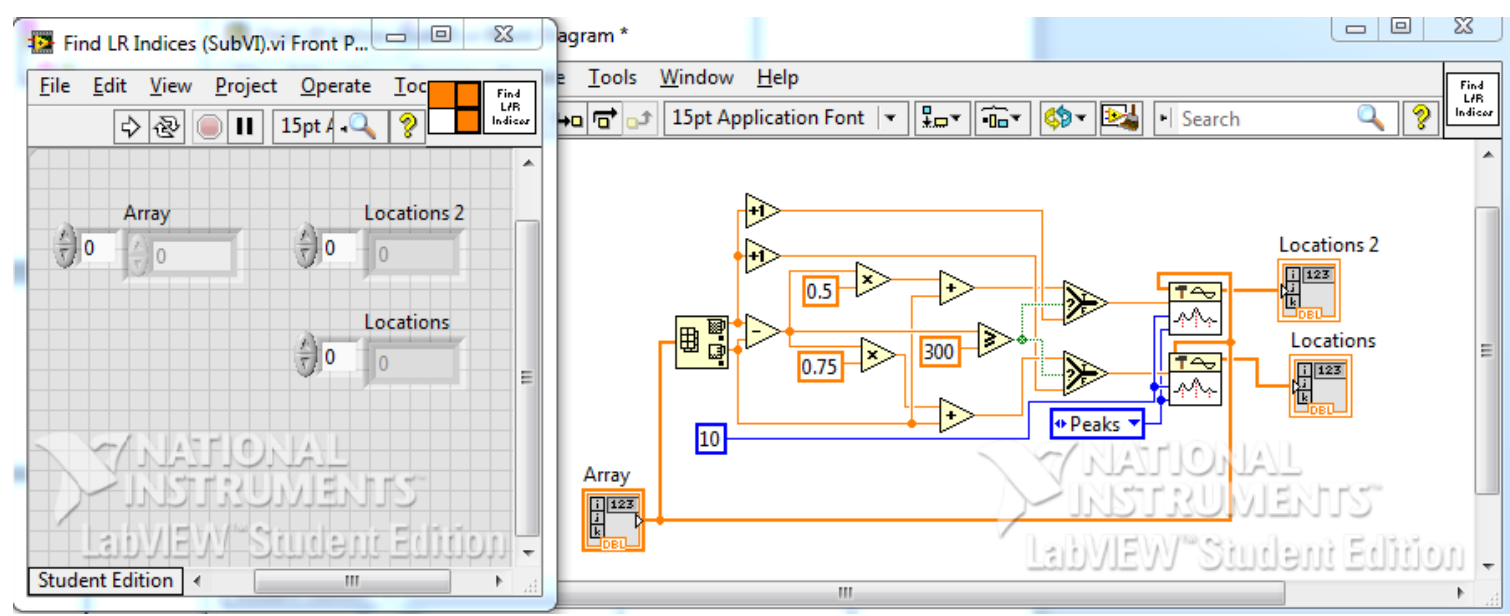

\section{Extract Right Indices (SubVI).vi}

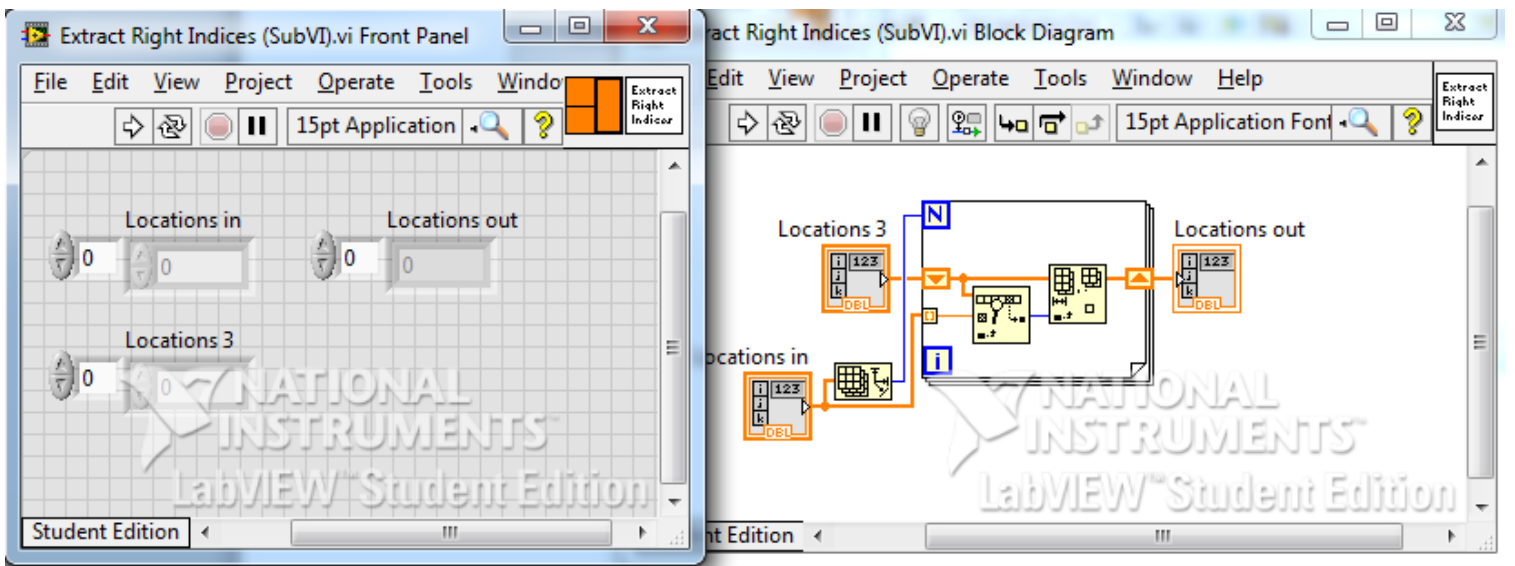


thesis_heart_rate.vi Front Panel

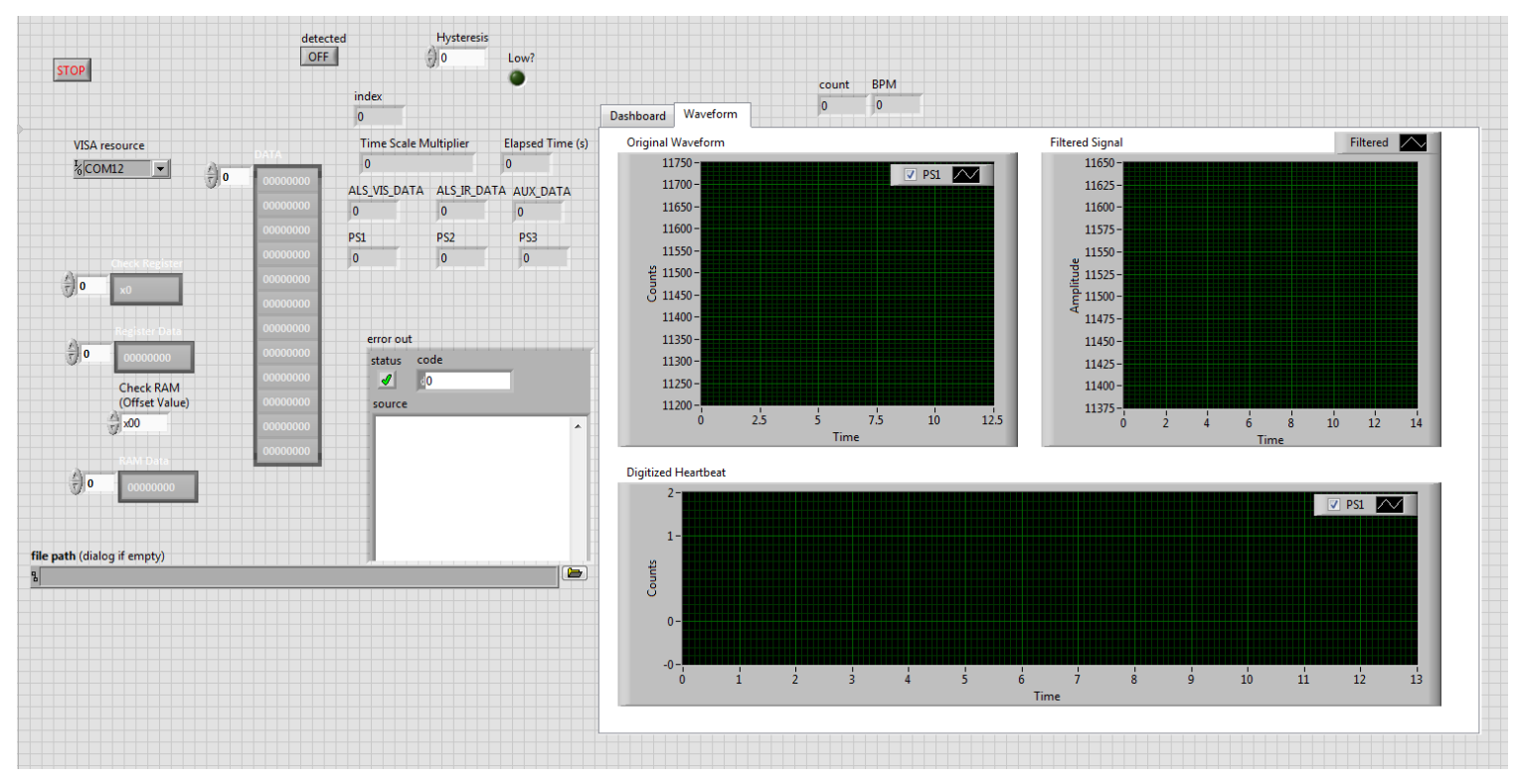

thesis_heart_rate.vi Block Diagram
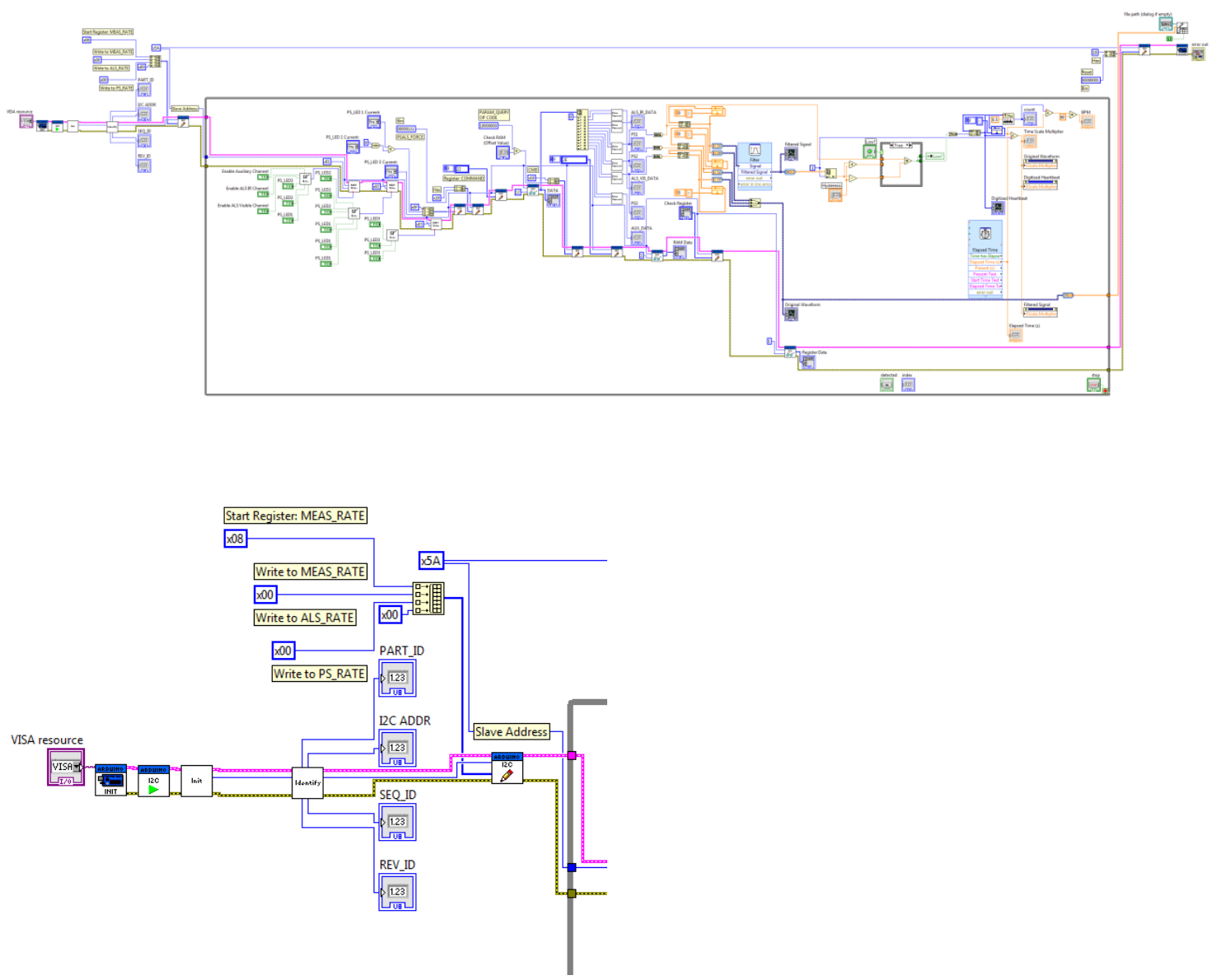

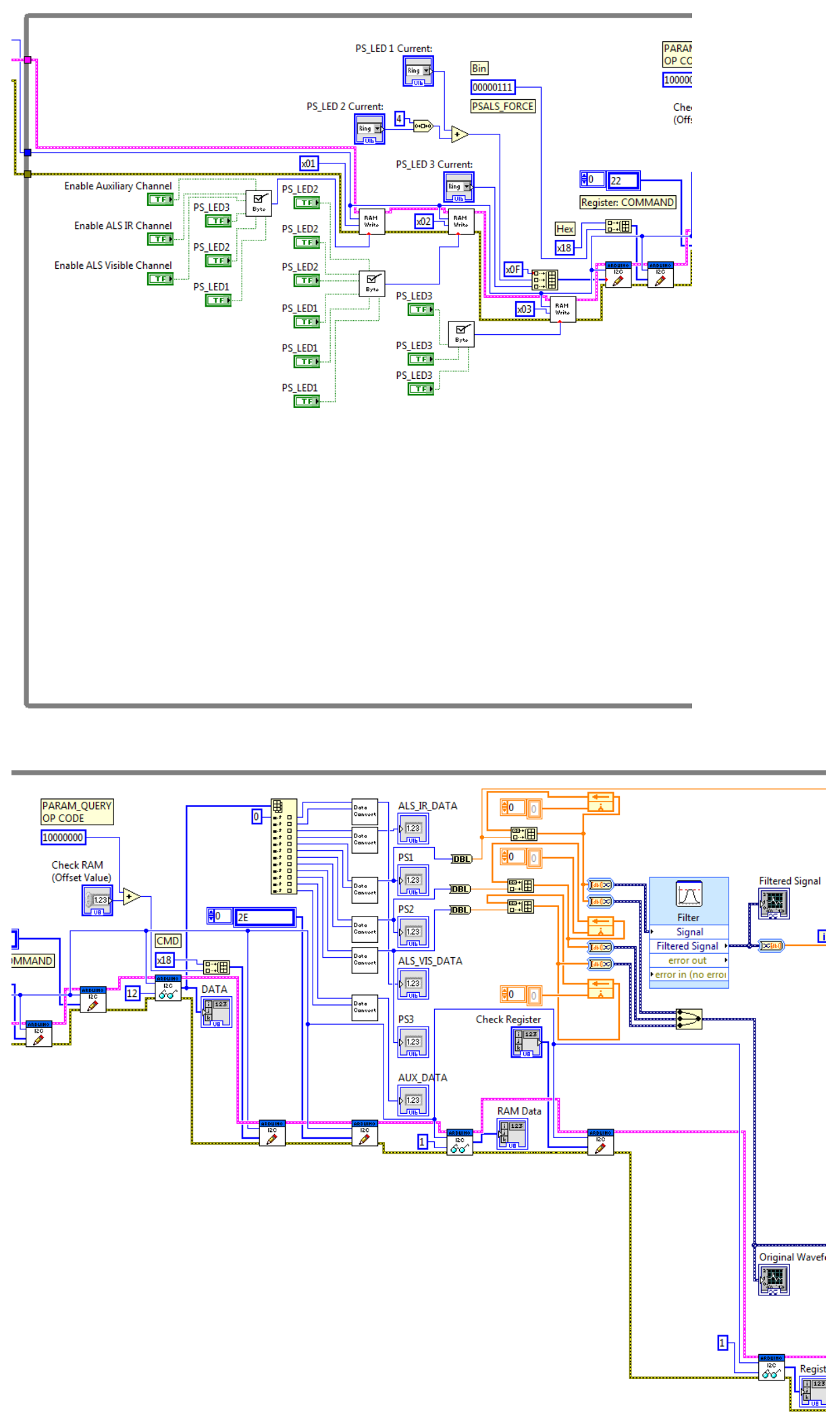

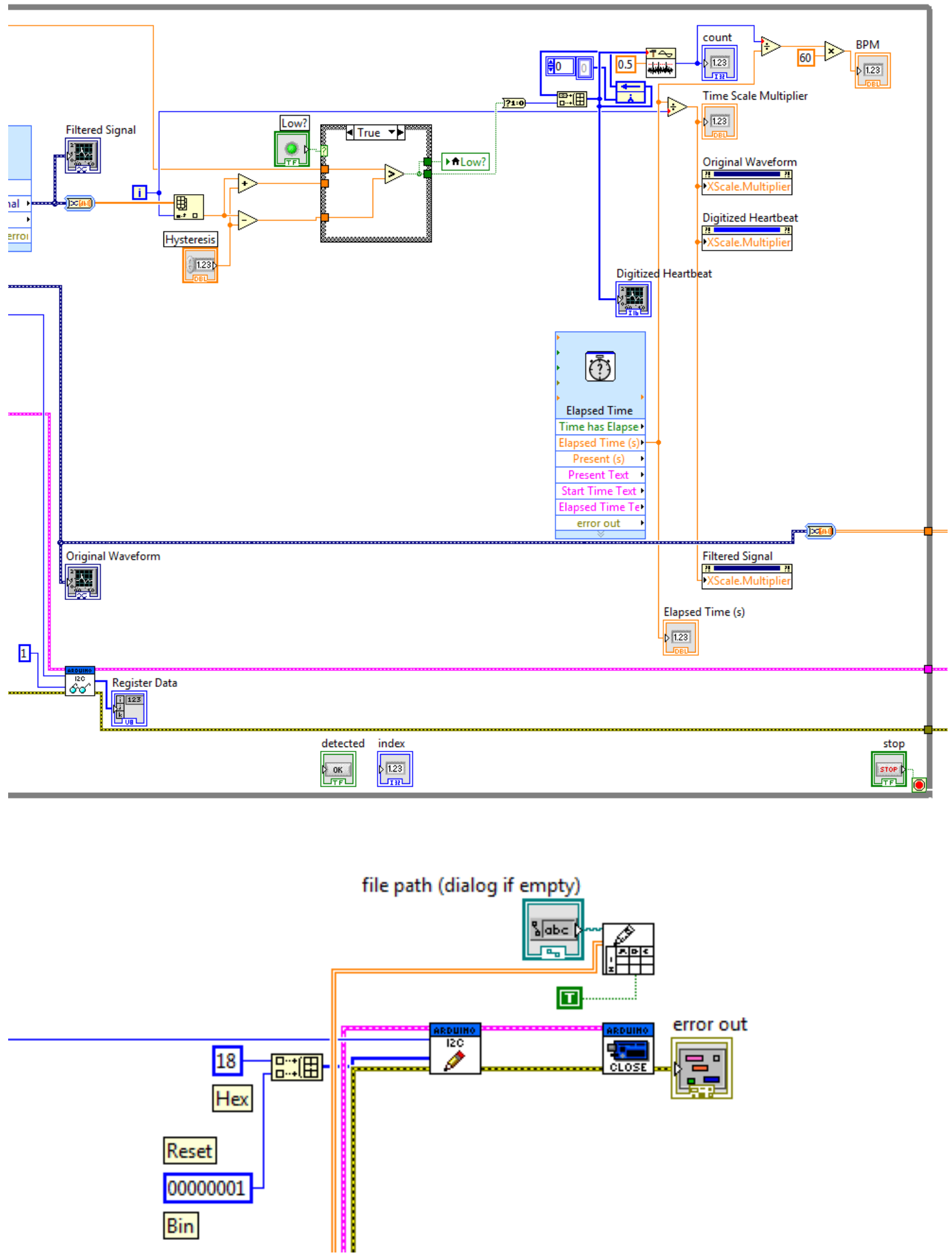


\section{APPENDIX C: I²C Protocol Explanation}

The $\mathrm{I}^{2} \mathrm{C}$ protocol is a communication protocol that enables multiple devices on one bus to communicate serially to each other. The devices are connected to two bidirectional lines, Serial Data Line (SDA) and Serial Clock (SCL) which are pulled high by some pull-up resistors. There are two types of devices on this bus line, the master and the slave. The host is usually the first to assert itself as the master by initiating the clock line. The master writes to the slaves by sending a start sequence where the data line is driven low while the clock line is still high as illustrated below.

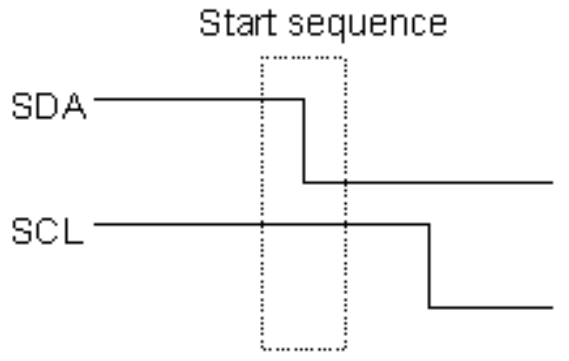

The master then sends the $\mathrm{I}^{2} \mathrm{C}$ address of the slave it wishes to address followed by a Read/Write low bit. The device send backs an acknowledge bit where it tells the master to continue sending data or to terminate any more data transfer as shown below.

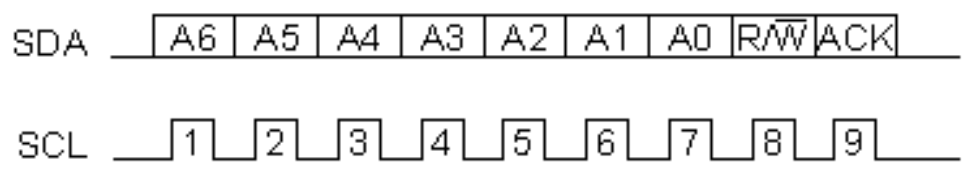

If the master is writing to the slave, the master will do so one byte at a time (where the first byte of data is the address of the internal register of the slave) and read 
the acknowledge bit from the slave after. All bytes that are sent after this one are the data bytes sent by the master.

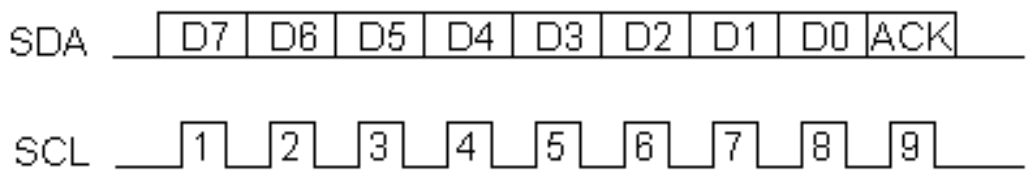

After the master is finished, it will send a stop sequence where the data line is drive high when the clock line is high as shown below.

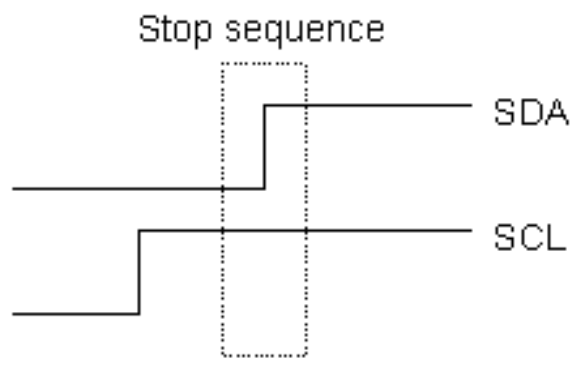


The read sequence of a slave starts off the same as the write sequence to it. The master will write the address of the slave followed by a low write bit. Following the acknowledge bit, the master will then write the address of the internal register of the slave it wishes to read from. After the acknowledge bit, the master will send a stop sequence and then send another start sequence. Some devices acknowledge a repeated start bit instead of a stop bit to continue. Next, the master writes slave address again but with a high read bit instead. The slave device acknowledges and starts sending the data one byte at a time to the master. The master acknowledges each data byte and sends a stop sequence when it is done reading. This sequence can be illustrated below.
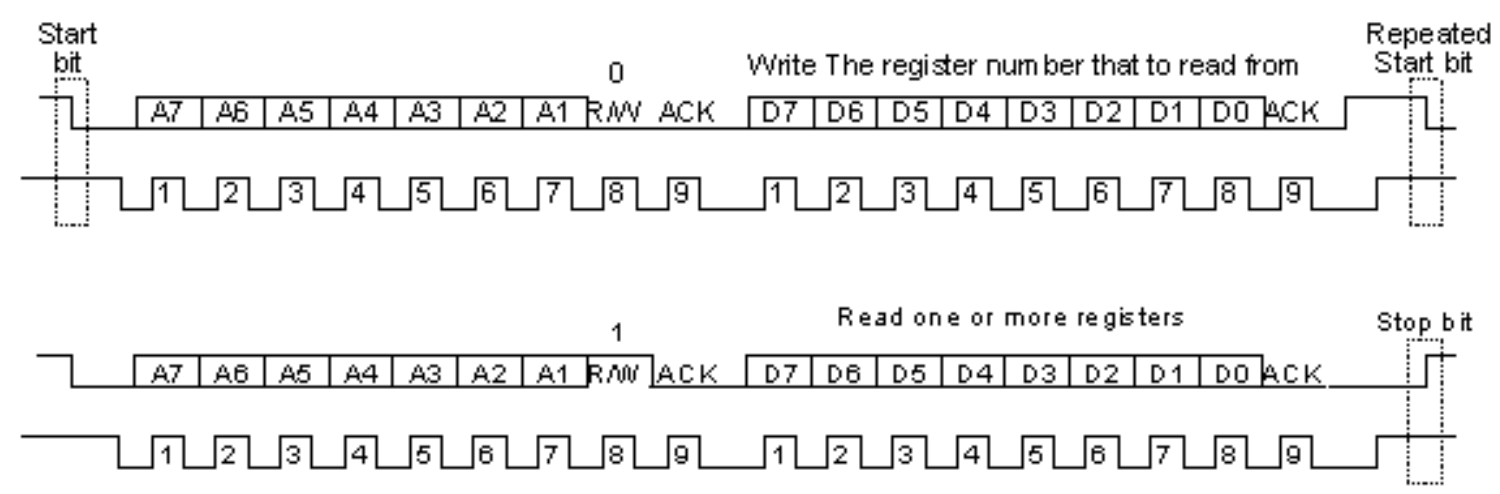